\title{
4- $\pi$-Photocyclization of 1,2-Dihydropyridazines: An Approach to Bicyclic 1,2-Diazetidines with Rich Synthetic Potential
}

\section{Supporting Information}

Thomas K. Britten, ${ }^{a}$ Paul D. Kemmitt, ${ }^{b}$ Nathan R. Halcovitch ${ }^{a}$ and

\author{
Susannah C. Coote $\mathrm{a}^{*}$
}

a Department of Chemistry, Lancaster University, Lancaster LA1 4YB, U.K.

b Medicinal Chemistry, Research and Early Development, Oncology R\&D, AstraZeneca, Cambridge CB10 1XL, U.K.

1. Synthetic Procedures and Analytical Data of New Compounds S2

1.1 General Information $\quad$ S2

1.2 UV/Vis Data for 1,2-Dihydropyridazine $\mathbf{2 b} \quad$ S3

1.3 General Procedure for Photocyclization S3

1.4 Synthetic Procedures $\quad$ S4

2. References for the Supporting Information $\quad$ S21

3. ${ }^{1} \mathrm{H} /{ }^{13} \mathrm{C}$ NMR Spectra of New Compounds $\quad$ S22

4. X-Ray Diffraction Data for 2d, 11d, 16 and $18 \quad S 79$

5. Differential Scanning Calorimetry (DSC) Data for 2d $S 85$ 


\section{Synthetic Procedures and Analytical Data of New Compounds}

\subsection{General Information}

Reagents were purchased in the highest purity available from Acros Organics, Alfa Aesar or Sigma Aldrich. Anhydrous solvents used in reactions were purchased from Acros Organics equipped with AcroSeal $^{\mathrm{TM}}$ and all other solvents used were of reagent grade. Reaction vessels were oven dried and cooled under an argon atmosphere prior to use and experiments were performed under argon gas. Reactions were monitored by thin-layer chromatography (TLC) and/or ${ }^{1} \mathrm{H}$ NMR spectroscopic analysis. Photochemical reactions were performed using a Rayonet RPR-100 Photochemical batch reactor.

Analytical TLC was carried out using Merck pre-coated aluminum-backed TLC silica gel plates (silica gel $\left.60 \mathrm{~F}_{254}\right)$ and the plates were visualised by UV light $(254 \mathrm{~nm})$ and by staining with either potassium permanganate or aqueous acidic ammonium molybdate(IV). Normal phase flash column chromatography on silica gel was carried out using silica gel from VWR (40-63 microns).

${ }^{1} \mathrm{H}$ NMR spectroscopic data were obtained on either 300 or $400 \mathrm{MHz}$ instruments and ${ }^{13} \mathrm{C}\left\{{ }^{1} \mathrm{H}\right\} \mathrm{NMR}$ data were obtained at $100 \mathrm{MHz}$ (Bruker Ultrashield 400 Plus) at $298 \mathrm{~K}$ unless otherwise specified. The chemical shifts are reported in parts per million $(\delta)$ relative to residual $\mathrm{CHCl}_{3}\left(\delta_{\mathrm{H}}=7.26 \mathrm{ppm}\right)$ and $\mathrm{CDCl}_{3}$ ( $\delta_{C}=77.16 \mathrm{ppm}$, central line), residual $d_{5}$-DMSO $\left(\delta_{H}=2.50 \mathrm{ppm}\right)$ and $d_{6}$-DMSO $\left(\delta_{\mathrm{C}}=39.52 \mathrm{ppm}\right.$, central line). The assignment of the signals in the ${ }^{1} \mathrm{H}$ and ${ }^{13} \mathrm{C}$ NMR spectra was achieved through 2D-NMR techniques: COSY, HSQC and HMBC. Coupling constants $(J)$ are quoted in Hertz. Infrared spectra were recorded on an Agilent Technologies Cary 630 FTIR spectrometer. Melting points were performed on a Sanyo Gallenkamp capillary melting point apparatus and are uncorrected. High resolution mass spectrometry data were recorded using electron spray ionization (ESI) or atmospheric pressure chemical ionization (APCI) on a Shimadzu LCMS-IT-TOF mass spectrometer. UV/Vis spectra were recorded using an Agilent Cary 60 UV-Vis spec spectrophotometer. For X-ray crystallography a suitable crystal was selected and mounted on a Mitegen loop using Paratone-N oil on a SuperNova, Dual, $\mathrm{Cu}$ at zero, AtlasS2 diffractometer. The crystal was kept at 100.2(5) K during data collection. Using Olex2,1 the structure was solved with the SheIXT structure solution program using direct methods and refined with the ShelXL refinement package using least squares minimisation. ${ }^{2,3}$ Figures and tables were prepared using Olex2 software. ${ }^{1}$

Bicycles $\mathbf{2 b}$ and $\mathbf{1 6}$ were submitted for thermal stability analysis (Process Safety Group, Pharmaceutical Technology and Development, AstraZeneca, Macclesfield, U. K.) using a Mettler differential scanning calorimeter (DSC). The sample crucible together with a reference crucible was heated to $500^{\circ} \mathrm{C}$ at $5 \mathrm{~K}$ /minute. Any heat generation (exotherm) or heat absorption (endotherm) was observed as a deviation from the baseline. Exothermic events that exceed $800 \mathrm{~J} / \mathrm{g}$ were considerd to indicate potential explosive properties. 


\subsection{UV/Vis data for 1,2-Dihydropyridazine $\mathbf{2 b}$}<smiles>CCOC(=O)N1C=CC=CN1C(=O)OCC</smiles>

2b

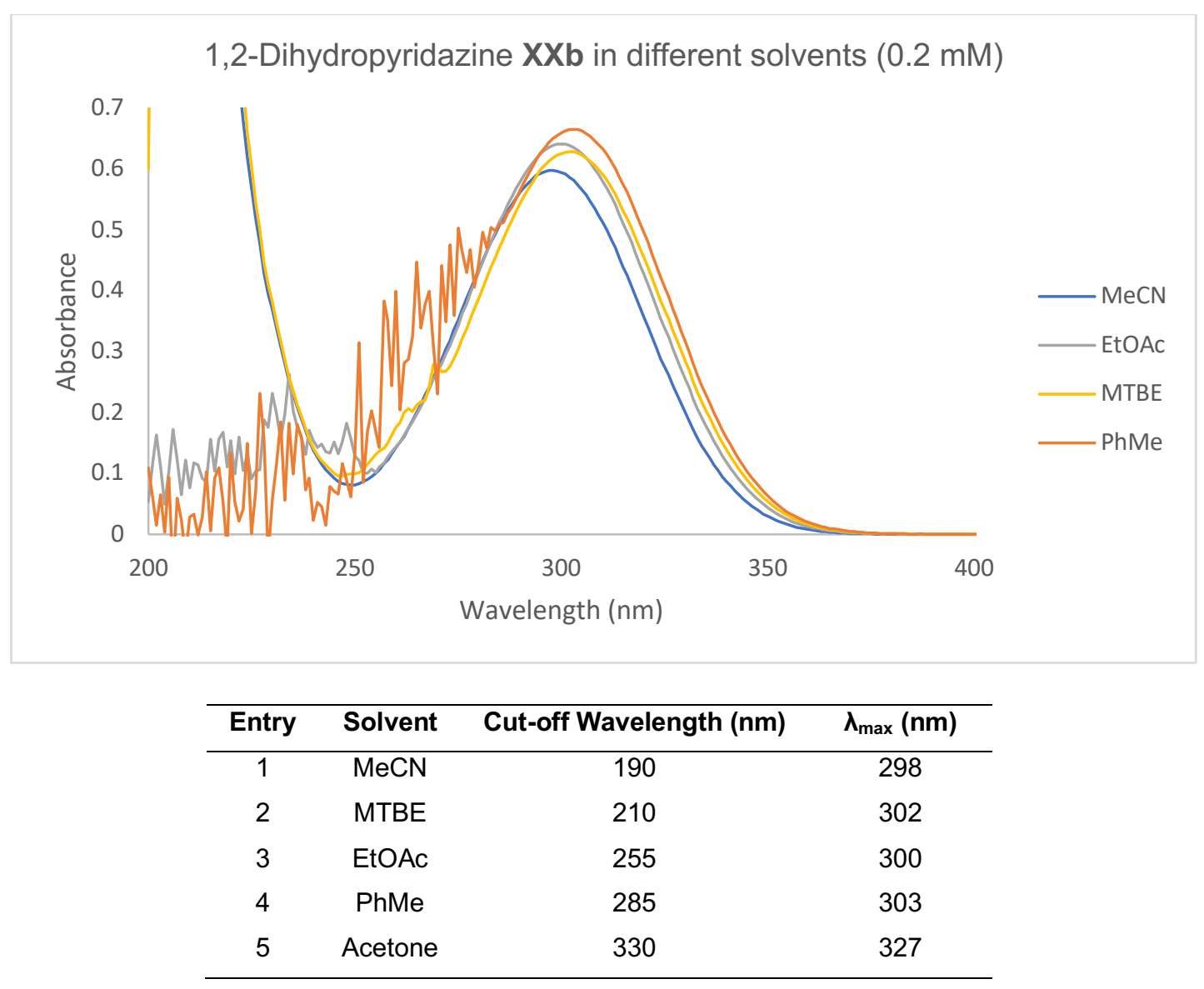

Table 1.1

\subsection{General Procedure for 4-m-Photocyclization}

A solution of 1,2-dihydropyridazine in either $\mathrm{MeCN}$ or $\mathrm{PhMe}(0.05 \mathrm{M} / 50 \mathrm{mM})$ was purged with argon for 15 minutes, then irradiated at room temperature $(\lambda=350 \mathrm{~nm})$ until complete consumption of starting material (24-44 hours). The solvent was evaporated under reduced pressure to give the crude product. The crude product was purified by flash column chromatography using an appropriate solvent system, as described for each individual procedure. 


\subsection{Synthetic Procedures}

\section{3-Methyl 1,2-dipropan-2-yl pyridazine-1,2,3-tricarboxylate S1}

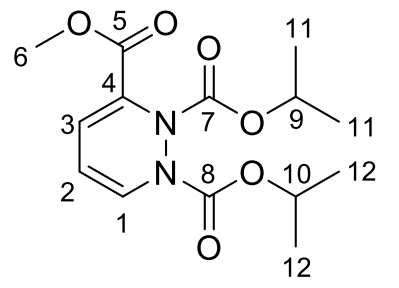

A solution of methyl 2-oxo-2H-pyran-3-carboxylate $(697 \mathrm{mg}, 4.52 \mathrm{mmol}$ ) and diisopropyl azodicarboxylate $(890 \mu \mathrm{L}, 4.52 \mathrm{mmol})$ in toluene $(10 \mathrm{~mL})$ was heated at $100{ }^{\circ} \mathrm{C}$ for $48 \mathrm{~h}$. After cooling to room temperature, the resulting solution was evaporated to give the crude product. Purification by flash column chromatography on silica gel (eluent $\mathrm{CH}_{2} \mathrm{Cl}_{2}-\mathrm{Et}_{2} \mathrm{O}$; 95:5) gave dihydropyridazine $\mathbf{S} 1$ (575 $\mathrm{mg}, 1.84 \mathrm{mmol}, 41 \%$ ) as a pale yellow oil.

$R_{f}\left(\mathrm{CH}_{2} \mathrm{Cl}_{2}-\mathrm{Et}_{2} \mathrm{O}, 95: 5\right)=0.20$

${ }^{1} \mathrm{H}$ NMR (298 K; $\left.400 \mathrm{MHz}, d_{6}-\mathrm{DMSO}\right): \delta 7.33$ (br d, J = 7.0 Hz, 1H, H1 or H3), 7.08-6.83 (br m, 1H, H1 or H3), 5.92-5.81 (br m, 1H, H2), 4.93 (sept, $J=6.2 \mathrm{~Hz}, 1 \mathrm{H}, \mathbf{H 9}$ or $\mathbf{H 1 0}$ ), 4.84 (sept, $J=6.2 \mathrm{~Hz}, 1 \mathrm{H}, \mathbf{H} \mathbf{9}$ or H10), 3.73 (s, 3H, H6), 1.25 (d, J = 6.2 Hz, 6H, H11 or H12), 1.15 (d, J = 6.2 Hz, 6H, H11 or H12). ${ }^{1} \mathrm{H}$ NMR (348 K; $\left.400 \mathrm{MHz}, d_{6}-\mathrm{DMSO}\right): \delta 7.29$ (d, $J=7.0 \mathrm{~Hz}, 1 \mathrm{H}, \mathbf{H 1}$ or H3), 6.95 (d, J = 5.6 Hz, 1H, H1 or H3), 5.86 (dd, $J=7.0,5.6 \mathrm{~Hz}, 1 \mathrm{H}, \mathbf{H 2}$ ), 4.95 (sept, $J=6.2 \mathrm{~Hz}, 1 \mathrm{H}, \mathbf{H 9}$ or H10), 4.86 (sept, $J=6.2$ $\mathrm{Hz}, 1 \mathrm{H}, \mathbf{H 9}$ or $\mathbf{H 1 0}$ ), 3.74 (s, 3H, H6), 1.28 (d, J = 6.2 Hz, 6H, H11 or H12), 1.27 (d, J = 6.2 Hz, 6H, H11 or H12), 1.19 (d, $J=6.2 \mathrm{~Hz}, 6 \mathrm{H}, \mathbf{H 1 1}$ or $\mathbf{H 1 2}$ ), 1.18 (d, $J=6.2 \mathrm{~Hz}, 6 \mathrm{H}, \mathbf{H 1 1}$ or $\mathbf{H 1 2}$ ).

${ }^{13} \mathrm{C}$ NMR (348 K; $100 \mathrm{MHz}, d_{6}$-DMSO): $\delta 161.8$ (C5), 153.9 (C7 or C8), 150.6 (C7 or C8), 133.1 (C1 or C3), 126.0 and 124.7 (C4, and C1 or C3), 108.2 (C2), 70.8 (C9 or C10), 70.7 (C9 or C10), 51.4 (C6), 21.15 (C11 or C12), 21.13 (C11 and C12), 20.96 (C11 or C12).

FTIR (ATR) v $\left(\mathrm{cm}^{-1}\right): 2984,1716(\mathrm{C}=\mathrm{O})$.

HRMS (ESI): $m / z$ calculated for: $\mathrm{C}_{14} \mathrm{H}_{20} \mathrm{~N}_{2} \mathrm{O}_{6}[\mathrm{M}+\mathrm{H}]^{+} 313.1394$, found 313.1406.

\section{Dimethyl 2,3-diazabicyclo[2.2.0]hex-5-ene-2,3-dicarboxylate $2 \mathrm{a}^{4,5}$}

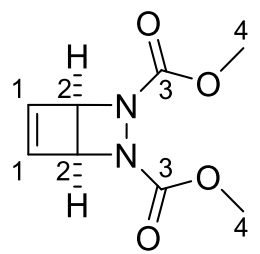

Using the general procedure, a solution of 1,2-dihydropyridazine 1a (140 mg, $0.71 \mathrm{mmol}$ ) in MeCN (14 $\mathrm{mL}$ ) was irradiated for 44 hours to give the crude product. Purification by flash column chromatography on silica gel (eluent: hexane-EtOAc, $4: 1 \rightarrow 2: 1)$ gave the bicycle $2 \mathrm{a}(61 \mathrm{mg}, 0.31 \mathrm{mmol}, 44 \%$ ) as an offwhite solid.

$R_{f}($ Hexane-EtOAc, 2:1) $=0.10$ 
$\mathrm{mp}=76-78{ }^{\circ} \mathrm{C}$

${ }^{1} \mathrm{H}$ NMR (400 MHz, CDCl 3 ): $\delta$ 6.74-6.71 (m, 2H, H1), 5.21-5.18 (m, 2H, H2), 3.81 (s, 6H, H4).

${ }^{13} \mathrm{C} \mathrm{NMR}\left(100 \mathrm{MHz}, \mathrm{CDCl}_{3}\right): \delta 160.6$ (C3), 143.7 (C1), 67.4 (C2), 53.7 (C4).

FTIR (ATR) v $\left(\mathrm{cm}^{-1}\right): 2961,1743(\mathrm{C}=\mathrm{O}), 1720(\mathrm{C}=\mathrm{O})$.

HRMS (ESI): $m / z$ calculated for: $\mathrm{C}_{8} \mathrm{H}_{10} \mathrm{~N}_{2} \mathrm{O}_{4}[\mathrm{M}+\mathrm{Na}]^{+} 221.0533$, found 221.0532.

\section{Diethyl 2,3-diazabicyclo[2.2.0]hex-5-ene-2,3-dicarboxylate $2 b^{6}$}

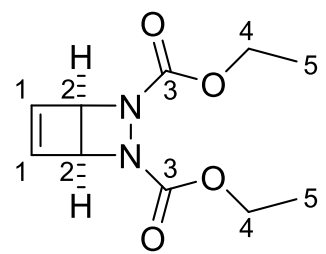

Using the general procedure, a solution of 1,2-dihydropyridazine $\mathbf{1 b}$ (170 $\mathrm{mg}, 0.75 \mathrm{mmol})$ in PhMe (15 $\mathrm{mL}$ ) was irradiated for 24 hours to give the crude product. Purification by flash column chromatography on silica gel (eluent: hexane-EtOAc, $7: 1 \rightarrow 4: 1)$ gave the bicycle $\mathbf{2 b}(121 \mathrm{mg}, 0.54 \mathrm{mmol}, 71 \%$ ) as a pale yellow oil.

$R_{f}($ Hexane-EtOAc, 1:1) $=0.29$

${ }^{1} \mathrm{H}$ NMR (400 MHz, CDCl 3 ): $\delta$ 6.74-6.71 (m, 2H, H1), 5.20-5.17 (m, 2H, H2), 4.30-4.19 (m, 4H, H4), $1.30(\mathrm{t}, \mathrm{J}=7.1 \mathrm{~Hz}, 6 \mathrm{H}, \mathrm{H5})$.

${ }^{13} \mathrm{C} \mathrm{NMR}\left(100 \mathrm{MHz}, \mathrm{CDCl}_{3}\right)$ : $\delta 160.1$ (C3), 143.6 (C1), 67.3 (C2), 62.8 (C4), 14.6 (C5).

FTIR $(A T R) \vee\left(\mathrm{cm}^{-1}\right): 2984,2935,1746(\mathrm{C}=0), 1703(\mathrm{C}=\mathrm{O})$.

HRMS (APCI): $\mathrm{m} / \mathrm{z}$ calculated for: $\mathrm{C}_{10} \mathrm{H}_{14} \mathrm{~N}_{2} \mathrm{O}_{4}[\mathrm{M}+\mathrm{H}]^{+} 227.1026$, found 227.1019.

\section{Ethyl 2-[(ethoxycarbonyl)amino]-1H-pyrrole-1-carboxylate 3b}

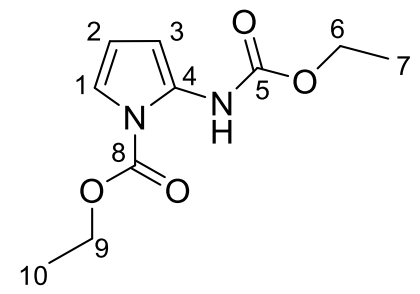

$R_{f}($ Hexane-EtOAc, 1:1) $=0.57$

${ }^{1} \mathrm{H}$ NMR (400 MHz, d6-DMSO): $\delta 8.92$ (br s, 1H, NH), 7.07 (dd, J = 3.6, $1.9 \mathrm{~Hz}, 1 \mathrm{H}, \mathrm{H1}$ ), 6.15 (t, $J=$ $3.6 \mathrm{~Hz}, 1 \mathrm{H}, \mathrm{H} 2$ ), 6.10-6.06 (br m, 1H, H3), 4.32 (q, J = 7.1 Hz, 2H, H9), 4.08 (q, J = 7.1 Hz, 2H, H6), $1.30(\mathrm{t}, J=7.1 \mathrm{~Hz}, 3 \mathrm{H}, \mathrm{H} 10), 1.20(\mathrm{t}, J=7.1 \mathrm{~Hz}, 3 \mathrm{H}, \mathrm{H} 7)$.

${ }^{13} \mathrm{C}$ NMR (100 MHz, d6-DMSO): $\delta 154.1$ (C5), 150.0 (C8), 128.1 (C4), 117.3 (C1), 110.1 (C2), 105.3 (C3), 63.4 (C9), 60.6 (C6), 14.5 (C7), 13.9 (C10).

FTIR (ATR) v $\left(\mathrm{cm}^{-1}\right): 3345(\mathrm{NH}), 3145(\mathrm{NH}), 2980,1718(\mathrm{C}=\mathrm{O})$.

HRMS (ESI): $m / z$ calculated for: $\mathrm{C}_{10} \mathrm{H}_{14} \mathrm{~N}_{2} \mathrm{O}_{4}[\mathrm{M}+\mathrm{H}]^{+} 227.1026$, found 227.1024.

Important NOE Contacts ( $\left.d_{6}-D M S O\right)$ : 


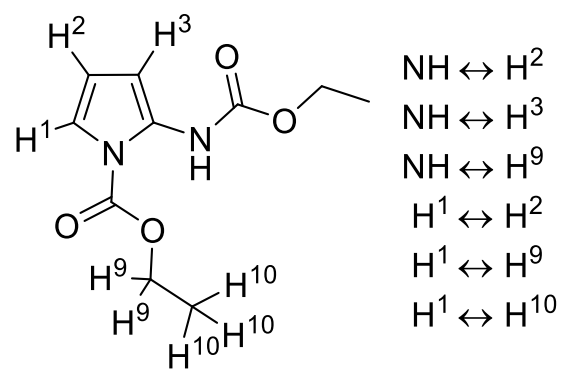

\section{Diisopropyl 2,3-diazabicyclo[2.2.0]hex-5-ene-2,3-dicarboxylate 2c}

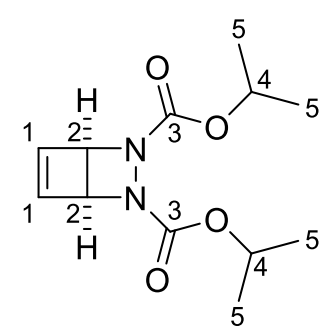

Using the general procedure, a solution of 1,2-dihydropyridazine 1c (191 mg, $0.75 \mathrm{mmol}$ ) in MeCN (15 $\mathrm{mL}$ ) was irradiated for 24 hours to give the crude product. Purification by flash column chromatography on silica gel (eluent: hexane-EtOAc, $9: 1 \rightarrow 4: 1)$ gave the bicycle $2 \mathrm{c}(159 \mathrm{mg}, 0.63 \mathrm{mmol}, 83 \%)$ as an offwhite solid.

$R_{f}($ Hexane-EtOAc, 1:1) $=0.35$

$\mathrm{mp}=48-50{ }^{\circ} \mathrm{C}$

${ }^{1} \mathrm{H}$ NMR (400 MHz, CDCl $)$ ): $\delta 6.72-6.69(\mathrm{~m}, 2 \mathrm{H}, \mathrm{H1}$ ), 5.17-5.14 (m, 2H, H2), 5.00 (sept, $J=6.3 \mathrm{~Hz}, 2 \mathrm{H}$, H4), 1.29-1.27 (m, 12H, H5).

${ }^{13} \mathrm{C}$ NMR (100 MHz, CDCl 3 ): $\delta 159.7$ (C3), 143.5 (C1), 70.6 (C4), 67.1 (C2), 21.1 (C5), 21.1 (C5). FTIR (ATR) v $\left(\mathrm{cm}^{-1}\right): 2988,2939,1698(\mathrm{C}=0)$.

HRMS (APCl): $m / z$ calculated for: $\mathrm{C}_{12} \mathrm{H}_{18} \mathrm{~N}_{2} \mathrm{O}_{4}[\mathrm{M}+\mathrm{Na}]^{+} 277.1159$, found 277.1147.

\section{Di-tert-butyl 2,3-diazabicyclo[2.2.0]hex-5-ene-2,3-dicarboxylate 2d}

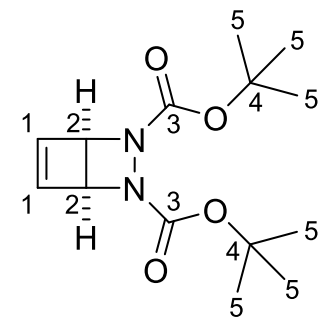

Using the general procedure, a solution of 1,2-dihydropyridazine $1 \mathrm{~d}$ (212 $\mathrm{mg}, 0.75 \mathrm{mmol})$ in $\mathrm{MeCN}$ (15 $\mathrm{mL}$ ) was irradiated for 24 hours to give the crude product. Purification by flash column chromatography on silica gel (eluent: hexane-EtOAc, $14: 1 \rightarrow 9: 1)$ gave the bicycle $\mathbf{2 d}(171 \mathrm{mg}, 0.61 \mathrm{mmol}, 81 \%)$ as an off-white solid. 
Scale up procedures

0.85 grams: Using general procedure $A$, a solution of 1,2-dihydropyridazine $1 \mathrm{~d}(0.85 \mathrm{~g}, 3.00 \mathrm{mmol})$ in MeCN (60 mL, $1 \times 60 \mathrm{~mL}$ tube) was irradiated for 48 hours to give the crude product. Purification by flash column chromatography on silica gel (eluent: hexane-EtOAc, $14: 1 \rightarrow 9: 1)$ gave the bicycle $\mathbf{2} \mathbf{d}(0.69$ $\mathrm{g}, 2.45 \mathrm{mmol}, 82 \%)$ as an off-white solid.

8.5 grams: Using general procedure A, a solution of 1,2-dihydropyridazine $1 \mathbf{d}(8.47 \mathrm{~g}, 30.0 \mathrm{mmol})$ in MeCN $(600 \mathrm{~mL}$ ) was split across $10 \times 60 \mathrm{~mL}$ tubes, then irradiated for 48 hours to give the crude product. Purification by flash column chromatography on silica gel (eluent: hexane-EtOAc, $14: 1 \rightarrow 9: 1$ ) gave the bicycle $\mathbf{2 d}(6.06 \mathrm{~g}, 21.5 \mathrm{mmol}, 72 \%)$ as an off-white solid.

$R_{f}($ Hexane-EtOAc, 1:1) $=0.46$

$\mathrm{mp}=81-83^{\circ} \mathrm{C}$

${ }^{1} \mathrm{H}$ NMR (400 MHz, CDCl 3 ): $\delta$ 6.72-6.69 (m, 2H, H1), 5.10-5.07 (m, 2H, H2), 1.49 (s, 18H, H5).

${ }^{13} \mathrm{C} \mathrm{NMR}\left(100 \mathrm{MHz}, \mathrm{CDCl}_{3}\right)$ : $\delta 159.0$ (C3), 143.5 (C1), 82.1 (C4), 66.7 (C2), 28.3 (C5).

FTIR (ATR) v $\left(\mathrm{cm}^{-1}\right): 2982,2937,1694(\mathrm{C}=\mathrm{O})$.

HRMS (APCl): $m / z$ calculated for: $\mathrm{C}_{14} \mathrm{H}_{22} \mathrm{~N}_{2} \mathrm{O}_{4}[\mathrm{M}+\mathrm{Na}]^{+} 305.1472$, found 305.1464.

\section{Dibenzyl 2,3-diazabicyclo[2.2.0]hex-5-ene-2,3-dicarboxylate 2e}

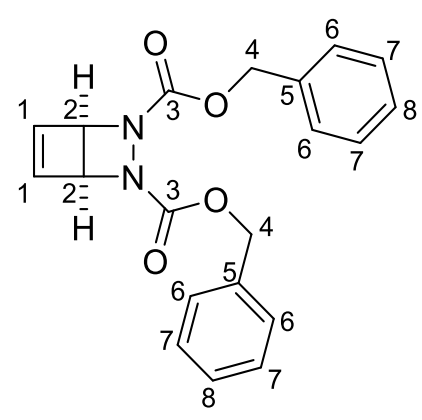

Using the general procedure, a solution of 1,2-dihydropyridazine $1 \mathrm{e}$ (263 mg, $0.75 \mathrm{mmol}$ ) in PhMe (15 $\mathrm{mL}$ ) was irradiated for 24 hours to give the crude product. Purification by flash column chromatography on silica gel (eluent: hexane-EtOAc, 9:1 $\rightarrow 4: 1)$ gave the bicycle $2 \mathrm{e}(155 \mathrm{mg}, 0.44 \mathrm{mmol}, 59 \%)$ as a yellow oil.

$R_{f}($ Hexane-EtOAc, $2: 1)=0.28$

${ }^{1} \mathrm{H} \mathrm{NMR}\left(400 \mathrm{MHz}, \mathrm{CDCl}_{3}\right):$ ठ 7.38-7.31 (m, 10H, H6, H7, H8), 6.67-6.64 (m, 2H, H1), 5.25-5.18 (m, 2H, H2, H4).

${ }^{13} \mathrm{C} \mathrm{NMR}(100 \mathrm{MHz}, \mathrm{CDCl}$ ) : $\delta 159.9$ (C3), 143.6 (C1), 135.7 (C5), 128.6 (C6 or C7), 128.4 (C8), 128.2

(C6 or C7), 68.2 (C4), 67.4 (C2).

FTIR (ATR) v $\left(\mathrm{cm}^{-1}\right)$ : 3032, 2954, $1703(\mathrm{C}=\mathrm{O})$.

HRMS (ESI): $m / z$ calculated for: $\mathrm{C}_{20} \mathrm{H}_{18} \mathrm{~N}_{2} \mathrm{O}_{4}[\mathrm{M}+\mathrm{Na}]^{+} 373.1159$, found 373.1142 . 


\section{Benzyl tert-butyl 2,3-diazabicyclo[2.2.0]hex-5-ene-2,3-dicarboxylate $2 f$}

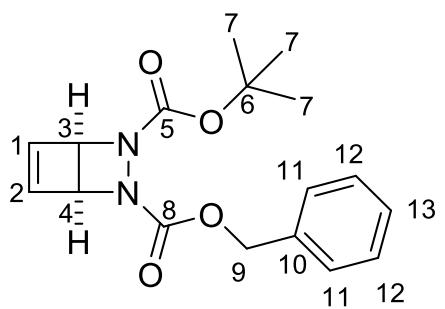

Using the general procedure, a solution of 1,2-dihydropyridazine 1 f $(237 \mathrm{mg}, 0.75 \mathrm{mmol})$ in PhMe (15 $\mathrm{mL}$ ) was irradiated for 24 hours to give the crude product. Purification by flash column chromatography on silica gel (eluent: hexane-EtOAc, $14: 1 \rightarrow 9: 1 \rightarrow 7: 1$ ) gave the bicycle $2 \mathrm{ff}(179 \mathrm{mg}, 0.57 \mathrm{mmol}, 75 \%$ ) as a colourless oil.

$R_{f}($ Hexane-EtOAc, 2:1) $=0.26$

${ }^{1} \mathrm{H}$ NMR (400 MHz, $\left.\mathrm{CDCl}_{3}\right)$ : $\delta$ 7.39-7.31 (m, 5H, H11, H12, H13), 6.70-6.69 (m, 1H, H1 or H2), 6.67$6.65(\mathrm{~m}, 1 \mathrm{H}, \mathbf{H 1}$ or $\mathbf{H 2}), 5.25-5.21(\mathrm{~m}, 2 \mathrm{H}, \mathbf{H} 9), 5.18-5.16(\mathrm{~m}, 1 \mathrm{H}, \mathbf{H} \mathbf{3}$ or $\mathbf{H 4}), 5.13-5.11(\mathrm{~m}, 1 \mathrm{H}, \mathbf{H} 3$ or H4), 1.46 (s, 9H, H7).

${ }^{13} \mathrm{C} \mathrm{NMR}$ (100 MHz, CDCl $)$ ): $\delta 159.9$ (C8), 158.9 (C5), 143.5 (C1 or C2), 143.5 (C1 or C2), 135.8 (C10), 128.6 (C11 or C12), 128.4 (C13), 128.2 (C11 or C12), 82.4 (C6), 68.0 (C9), 67.1, (C3 or C4), 67.1 (C3 or $\mathbf{C 4}), 28.2(\mathbf{C} 7)$.

FTIR (ATR) v $\left(\mathrm{cm}^{-1}\right):$ 2978, 2932, $1735(\mathrm{C}=0), 1702(\mathrm{C}=0)$.

HRMS (ESI): $m / z$ calculated for: $\mathrm{C}_{17} \mathrm{H}_{20} \mathrm{~N}_{2} \mathrm{O}_{4}[\mathrm{M}+\mathrm{Na}]^{+} 339.1315$, found 339.1310 .

tert-Butyl methyl 2,3-diazabicyclo[2.2.0]hex-5-ene-2,3-dicarboxylate 2g

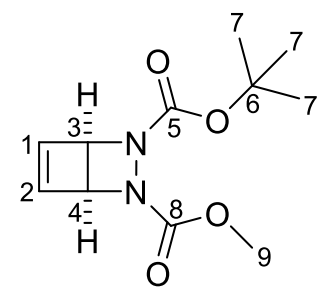

Using the general procedure, a solution of 1,2-dihydropyridazine $1 \mathbf{g}(180 \mathrm{mg}, 0.75 \mathrm{mmol})$ in PhMe (15 $\mathrm{mL}$ ) was irradiated for 24 hours to give the crude product. Purification by flash column chromatography on silica gel (eluent: hexane-EtOAc, 4:1) gave the bicycle $\mathbf{2} \mathbf{g}(146 \mathrm{mg}, 0.61 \mathrm{mmol}, 81 \%)$ as an off-white solid.

$R_{f}($ Hexane-EtOAc, $2: 1)=0.18$

$\mathrm{mp}=79-81^{\circ} \mathrm{C}$

${ }^{1} \mathrm{H}$ NMR (400 MHz, CDCl 3 ): $\delta$ 6.74-6.72 (m, 1H, $\mathbf{H 1}$ or $\mathbf{H 2}$ ), 6.70-6.69 (m, 1H, H1 or H2), 5.17-5.16 (m, $1 \mathrm{H}, \mathbf{H} 3$ or H4), 5.12-5.10 (m, 1H, H3 or H4), 3.79 (s, 3H, H9), 1.49 (s, 9H, H7).

${ }^{13} \mathrm{C}$ NMR (100 MHz, $\mathrm{CDCl}_{3}$ ): $\delta 160.6$ (C8), 158.9 (C5), 143.6 (C1 or C2), 143.4 (C1 or C2), 82.5 (C6), 67.2 (C3 or C4), 67.0 (C3 or C4), 53.6 (C9), 28.3 (C7).

FTIR (ATR) v $\left(\mathrm{cm}^{-1}\right): 2978,2932,1735(\mathrm{C}=0), 1702(\mathrm{C}=0)$.

HRMS (ESI): $m / z$ calculated for: $\mathrm{C}_{11} \mathrm{H}_{16} \mathrm{~N}_{2} \mathrm{O}_{4}[\mathrm{M}+\mathrm{Na}]^{+} 263.1002$, found 263.0991 . 
1-Methyl 2,3-dipropan-2-yl 2,3-diazabicyclo[2.2.0]hex-5-ene-1,2,3-tricarboxylate $2 \mathrm{~h}$

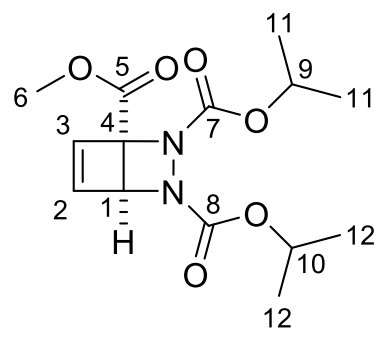

Using the general procedure, a solution of 1,2-dihydropyridazine $1 \mathrm{~h}$ (234 $\mathrm{mg}, 0.75 \mathrm{mmol})$ in PhMe (15 $\mathrm{mL}$ ) was irradiated for 12 hours to give the crude product. Purification by flash column chromatography on silica gel (eluent: hexane-EtOAc, 2:1) gave the bicycle $\mathbf{2 h}(185 \mathrm{mg}, 0.59 \mathrm{mmol}, 80 \%)$ as a yellow oil. $R_{f}($ Hexane-EtOAc, 2:1) $=0.25$

${ }^{1} \mathrm{H} \mathrm{NMR}\left(400 \mathrm{MHz}, \mathrm{CDCl}_{3}\right): \delta 6.85$ (d, J = 2.6 Hz, 1H, H3), 6.71 (dd, J=3.3 Hz, 2.6 Hz, 1H, H2), 5.14 (d, $J=3.3 \mathrm{~Hz}, 1 \mathrm{H}, \mathbf{H 1}$ ), 5.06-4.93 (m, 2H, H9 and H10), 3.79 (s, 3H, H6), 1.30-1.25 (m, 12H, H11 and H12).

${ }^{13} \mathrm{C}$ NMR (100 MHz, CDCl $)$ : $\delta 166.0$ (C6), 159.2 and 158.6 (C7 and C8), 143.8 and 142.0 (C2 and C3), 74.0 (C4), 71.17 and 71.12 (C9 and C10), 67.7 (C1), 53.0 (C6), 22.05 and 21.99 (C11 and C12). FTIR (ATR) v $\left(\mathrm{cm}^{-1}\right): 2978,2932,1735(\mathrm{C}=\mathrm{O}), 1702(\mathrm{C}=\mathrm{O})$.

HRMS (ESI): $m / z$ calculated for: $\mathrm{C}_{14} \mathrm{H}_{21} \mathrm{~N}_{2} \mathrm{O}_{6}[\mathrm{M}+\mathrm{Na}]^{+} 313.1394$, found 313.1386 .

\section{1,2-Bis(tert-butoxycarbonyl)-1,2-diazetidine-3,4-dicarboxylic acid 4}<smiles></smiles>

An aqueous $10 \% \mathrm{NalO}_{4}$ solution $(20 \mathrm{~mL})$ was added dropwise to a stirred solution of bicyclic 1,2diazetidine 2d (402 mg, $1.42 \mathrm{mmol}, 1.0 \mathrm{eq}$ ) and $\mathrm{RuO}_{2} \cdot \mathrm{xH}_{2} \mathrm{O}(2 \mathrm{mg}, 0.01 \mathrm{mmol}, 0.01 \mathrm{eq}$ ) in EtOAc (14 $\mathrm{mL}$ ) at $0{ }^{\circ} \mathrm{C}$, then stirred at room temperature for 41 hours. The organic layer was separated, the aqueous layer was saturated with $\mathrm{NaCl}$ and extracted with EtOAc $(5 \times 10 \mathrm{~mL})$. Isopropanol $(2 \mathrm{~mL})$ was added to the combined organic layers and left to stand for 2 hours. The organic layer was dried $\left(\mathrm{MgSO}_{4}\right)$, filtered through Celite and evaporated under reduced pressure to give diacid 4 (374 mg, 1.08 $\mathrm{mmol}, 76 \%)$ as a grey solid, which was used without further purification.

$R_{f}\left(\mathrm{CH}_{2} \mathrm{Cl}_{2}-\mathrm{MeOH}, 9: 1\right)=$ baseline

$\mathrm{mp}=168-170{ }^{\circ} \mathrm{C}$ (decomposition)

${ }^{1} \mathrm{H}$ NMR (400 MHz, CDCl 3 ): $\delta 10.6$ (br s, 2H, OH), 5.00 (br s, 2H, H2), 1.46 (s, 18H, H5). 
$\left.{ }^{13} \mathrm{C} \mathrm{NMR} \mathrm{(100} \mathrm{MHz,} \mathrm{CDCl}\right)$ : $\delta 170.8$ (C1), 158.4 (C3), 84.2 (C4), 61.6 (C2), 28.0 (C5).

FTIR (ATR) v ( $\left.\mathrm{cm}^{-1}\right): 3078(\mathrm{OH}), 2980,2935,1718(\mathrm{C}=\mathrm{O})$.

HRMS (ESI): $m / z$ calculated for: $\mathrm{C}_{14} \mathrm{H}_{22} \mathrm{~N}_{2} \mathrm{O}_{8}[\mathrm{M}+\mathrm{Na}]^{+} 369.1268$, found 369.1254 .

\section{1,2-Di-tert-butyl 3,4-dimethyl 1,2-diazetidine-1,2,3,4-tetracarboxylate 5}<smiles>COC(=O)C1C(C(=O)OI)N(C(=O)OC(C)(C)C)N1C(=O)OC(C)(C)C</smiles>

An aqueous $10 \% \mathrm{NalO}_{4}$ solution $(20 \mathrm{~mL})$ was added dropwise to a stirred solution of bicyclic 1,2diazetidine 2d (402 mg, $1.42 \mathrm{mmol}, 1.0 \mathrm{eq}$ ) and $\mathrm{RuO}_{2} . \mathrm{xH}_{2} \mathrm{O}(2 \mathrm{mg}, 0.01 \mathrm{mmol}, 0.01 \mathrm{eq}$ ) in EtOAc (14 $\mathrm{mL}$ ) at $0{ }^{\circ} \mathrm{C}$, then stirred at room temperature for 72 hours. The organic layer was separated and the aqueous layer was saturated with $\mathrm{NaCl}$ and extracted with EtOAc $(5 \times 10 \mathrm{~mL})$. Isopropanol $(2 \mathrm{~mL})$ was added to the combined organic layers and left to stand for 2 hours. The organic layer was dried $\left(\mathrm{MgSO}_{4}\right)$, filtered through Celite and evaporated under reduced pressure to give the crude diacid 4 , which was taken up in $\mathrm{MeOH}(10 \mathrm{~mL})$. (Trimethylsilyl)diazomethane $(2 \mathrm{M}$ solution in hexanes, $2.1 \mathrm{~mL}$, $4.26 \mathrm{mmol}, 3 \mathrm{eq}$ ) was added dropwise to the resulting stirred solution of diacid 4 at room temperature, and the resulting mixture was stirred for 10 minutes. The solvent was removed under reduced pressure. The residue was dissolved in $\mathrm{CHCl}_{3}(20 \mathrm{~mL})$, washed with a saturated solution of sodium thiosulfate $(10 \mathrm{~mL})$, dried $\left(\mathrm{Na}_{2} \mathrm{SO}_{4}\right)$ and the solvent was removed under reduced pressure to give the crude product. Purification by flash column chromatography on silica gel (eluent: hexane-EtOAc, 4:1) gave the diester 5 as a colourless film (389 $\mathrm{mg}, 1.04 \mathrm{mmol}, 73 \%$ over two steps).

$R_{f}($ Hexane-EtOAc, $1: 1)=0.48$

${ }^{1} \mathrm{H}$ NMR (400 MHz, CDCl $)$ : $\delta 4.94$ (s, 2H, H3), 3.76 (s, 6H, H1) 1.46 (s, 18H, H6).

${ }^{13} \mathrm{C} \mathrm{NMR}\left(100 \mathrm{MHz}, \mathrm{CDCl}_{3}\right.$ ): $\delta 167.7$ (C2), 158.0 (C4), 83.2 (C5), 61.4 (C3), 52.9 (C1), 28.1 (C6).

FTIR (ATR) v ( $\left.\mathrm{cm}^{-1}\right):$ 2980, 1752 (C=Oester), 1709 (C=Ocarbamate).

HRMS (ESI): $m / z$ calculated for: $\mathrm{C}_{16} \mathrm{H}_{26} \mathrm{~N}_{2} \mathrm{O}_{8}[\mathrm{M}+\mathrm{Na}]^{+} 397.1581$, found 397.1585; [2M+Na] ${ }^{+} 771.3271$, found 771.3271 .

1-tert-Butyl 2,3,4-trimethyl 1,2-diazetidine-1,2,3,4-tetracarboxylate 6<smiles>COC(=O)[C@@H]1[C@@H](C(=O)OC)N(C(=O)OC(C)(C)C)N1C(=O)O</smiles>

An aqueous $10 \% \mathrm{NalO}_{4}$ solution $(5 \mathrm{~mL})$ was added dropwise to a stirred solution of bicyclic 1,2diazetidine 2d (117 mg, $0.49 \mathrm{mmol}, 1.0 \mathrm{eq}$ ) and $\mathrm{RuO}_{2} . \mathrm{xH}_{2} \mathrm{O}(3 \mathrm{mg}, 0.02 \mathrm{mmol}, 0.04 \mathrm{eq})$ in EtOAc (5 
$\mathrm{mL}$ ) at $0{ }^{\circ} \mathrm{C}$, then stirred at room temperature for 72 hours. The organic layer was separated, the aqueous layer was saturated with $\mathrm{NaCl}$ and extracted with EtOAc $(5 \times 10 \mathrm{~mL})$. Isopropanol $(2 \mathrm{~mL})$ was added to the combined organic layers and left to stand for 2 hours. The organic layer was dried $\left(\mathrm{MgSO}_{4}\right)$, filtered through Celite and evaporated under reduced pressure to give diacid $\mathbf{6}$ as a grey solid, which was used in the next step without further purification. (Trimethylsilyl)diazomethane (2M solution in hexanes, $2.0 \mathrm{~mL}, 2.00 \mathrm{mmol}, 4.1 \mathrm{eq}$ ) was added dropwise to a stirred solution of diacid 6 in $\mathrm{MeOH}$ $(5 \mathrm{~mL})$ at room temperature, then stirred for 10 minutes. The solvent was removed under reduced pressure. The residue was dissolved in $\mathrm{CHCl}_{3}(2 \mathrm{~mL})$, washed with a saturated solution of sodium thiosulfate $(5 \mathrm{~mL})$, dried $\left(\mathrm{Na}_{2} \mathrm{SO}_{4}\right)$ and the solvent removed under reduced pressure to give the crude product. Purification by flash column chromatography on silica gel (eluent: hexane-EtOAc, 1:1) gave the diester 6 as a colourless oil ( $162 \mathrm{mg}, 0.37 \mathrm{mmol}, 75 \%$ over two steps).

$R_{f}($ Hexane-EtOAc, 1:1) $=0.35$

${ }^{1} \mathrm{H}$ NMR $\left(400 \mathrm{MHz}, \mathrm{CDCl}_{3}\right): \delta 5.04-4.98(\mathrm{~m}, 2 \mathrm{H}, \mathrm{H} 3$ and $\mathbf{H} 4), 3.81(\mathrm{~s}, 3 \mathrm{H}, \mathrm{H} 11), 3.78(\mathrm{~s}, 6 \mathrm{H}, \mathrm{H} 1$ and H6), 1.47 (s, 9H, H9).

${ }^{13} \mathrm{C}$ NMR (100 MHz, CDCl $)$ ): $\delta 167.5$ (C2 and C5), 160.0 (C10), 157.7 (C7), 83.7 (C8), 61.8 (C3 or C4), 61.4 (C3 or C4), 54.0 (C11), 53.1 (C1 or C6), 53.0 (C1 or C6), 28.1 (C9).

FTIR (ATR) v $\left(\mathrm{cm}^{-1}\right):$ 2958, $1735(\mathrm{C}=\mathrm{O})$.

HRMS (ESI): $m / z$ calculated for: $\mathrm{C}_{13} \mathrm{H}_{20} \mathrm{~N}_{2} \mathrm{O}_{8}[\mathrm{M}+\mathrm{Na}]^{+} 355.1112$, found 355.1117 .

\section{Di-tert-butyl 3,4-diethenyl-1,2-diazetidine-1,2-dicarboxylate 7}

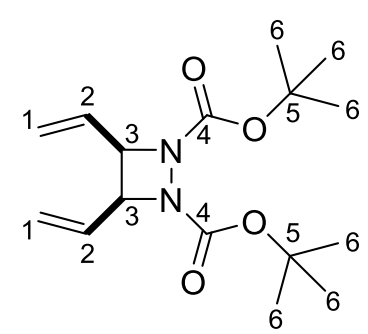

Ethylene was bubbled (10 s) through a stirred solution of bicyclic 1,2-diazetidine $\mathbf{2 g}(44 \mathrm{mg}, 0.18 \mathrm{mmol}$, 1.0 eq) and Hoveyda-Grubbs $2^{\text {nd }}$ generation catalyst $(6 \mathrm{mg}, 0.01,0.04 \mathrm{eq})$ in $\mathrm{CH}_{2} \mathrm{Cl}_{2}(2 \mathrm{~mL})$ at room temperature under nitrogen. The reaction was stirred at room temperature under ethylene atmosphere for 30 mins then the solvent was removed under reduced pressure to give the crude product. Purification by flash column chromatography on silica gel (eluent: hexane-EtOAc, 5:1) gave the 1,2-diazetidine 7 (42 mg, $0.14 \mathrm{mmol}, 76 \%$ ) as a colourless oil.

$R_{f}($ Hexane-EtOAc, 5:1) $=0.28$

${ }^{1} \mathrm{H}$ NMR (400 MHz, $\left.\mathrm{CDCl}_{3}\right): \delta$ 5.92-5.80 (m, 2H, H2), 5.39-5.33 (m, 4H, H1), 4.89-4.84 (m, 2H, H3), $1.45(\mathrm{~s}, 18 \mathrm{H}, \mathrm{H6})$.

$\left.{ }^{13} \mathrm{C} \mathrm{NMR} \mathrm{(100} \mathrm{MHz,} \mathrm{CDCl}\right)$ : $\delta 158.7$ (C5), 132.5 (C2), 120.8 (C1), 82.0 (C5), 66.6 (C3), 28.3 (C6).

FTIR (ATR) v $\left(\mathrm{cm}^{-1}\right):$ 2978, $1703(\mathrm{C}=\mathrm{O})$.

HRMS (ESI): $m / z$ calculated for: $\mathrm{C}_{16} \mathrm{H}_{26} \mathrm{~N}_{2} \mathrm{O}_{4}[\mathrm{M}+]^{+} 333.1785$, found 333.1783 . 
tert-Butyl methyl 3,4-diethenyl-1,2-diazetidine-1,2-dicarboxylate 8<smiles>C=CC1C(C=C)N(C(=O)OC(C)(C)C)N1C(=O)O</smiles>

Ethylene was bubbled (10 s) through a stirred solution of bicyclic 1,2-diazetidine $\mathbf{2 d}$ ( $50 \mathrm{mg}, 0.18 \mathrm{mmol}$, 1.0 eq) and Hoveyda-Grubbs $2^{\text {nd }}$ generation catalyst (6 mg, $\left.0.01,0.04 \mathrm{eq}\right)$ in $\mathrm{CH}_{2} \mathrm{Cl}_{2}(2 \mathrm{~mL})$ at room temperature under nitrogen. The reaction was stirred at room temperature under ethylene atmosphere for $1 \mathrm{~h}$, after which time further catalyst $(6 \mathrm{mg})$ was added. After stirring for a further $1 \mathrm{~h}$, the solvent was removed under reduced pressure to give the crude product. Purification by flash column chromatography on silica gel (eluent: hexane-EtOAc, 5:1 $\rightarrow 3: 1$ ) gave the 1,2-diazetidine 8 (39 mg, 0.15 $\mathrm{mmol}, 79 \%$ ) as a colourless oil.

$R_{f}($ Hexane-EtOAc, 3:1) $=0.24$

${ }^{1} \mathrm{H}$ NMR (400 MHz, CDCl$)$ ): $\delta$ 5.90-5.79 (m, 2H, H), 5.44-5.34 (m, 4H, H), 4.98-4.89 (m, 2H, H), 3.76 (s, 3H, H11), 1.46 (s, 9H, H9).

${ }^{13} \mathrm{C}$ NMR (100 MHz, CDCl 3 ): $\delta 160.2$ (C10), 158.5 (C7), 132.3 (C2 or C5), 131.9 (C2 or C5), 121.0 (C1 and C6), 82.3 (C8), 67.0 (C3 or C4), 66.7 (C3 or C4), 53.3 (C11), 28.3 (C9).

FTIR (ATR) v $\left(\mathrm{cm}^{-1}\right): 2980,1709(\mathrm{C}=0)$.

HRMS (ESI): $m / z$ calculated for: $\mathrm{C}_{13} \mathrm{H}_{20} \mathrm{~N}_{2} \mathrm{O}_{4}[\mathrm{M}+]^{+} 291.1315$, found 291.1306.

\section{Di-tert-butyl 3-ethenyl-4-[(E/Z)-2-phenylethenyl]-1,2-diazetidine-1,2-dicarboxylate 9a,b}<smiles>C=CC1C(/C=C/c2ccccc2)N(C(=O)OC(C)(C)C)N1C(=O)OC(C)(C)C</smiles>

Styrene $(61 \mu \mathrm{L}, 0.53 \mathrm{mmol}, 5.0 \mathrm{eq})$ was added in one portion to a stirred solution of bicyclic 1,2diazetidine $2 \mathrm{~d}$ (30 mg, $0.11 \mathrm{mmol}, 1.0 \mathrm{eq}$ ) and Hoveyda-Grubbs $2^{\text {nd }}$ generation catalyst ( $3 \mathrm{mg}, 0.01$, $0.05 \mathrm{eq}$ ) in $\mathrm{CH}_{2} \mathrm{Cl}_{2}$ at room temperature under argon, then heated at reflux for 1 hour. The reaction mixture was cooled to room temperature and the solvent removed under reduced pressure to give the crude product. Purification by flash column chromatography on silica gel (eluent: hexane-EtOAc, $11: 1 \rightarrow 7: 1)$ gave the 1,2-diazetidine 9 a,b (30 $\mathrm{mg}, 0.08 \mathrm{mmol}, 73 \%, E: Z 1.0: 1.5)$ as a light brown oil. 


\section{$\underline{\text { Z-isomer 9a }}$}

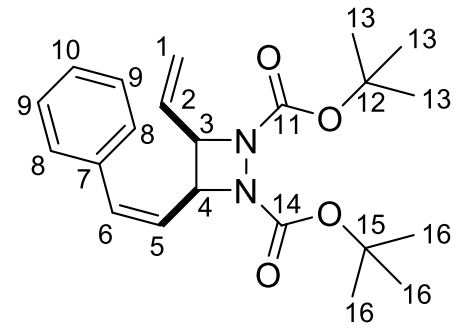

$R_{f}($ Hexane-EtOAc, 2:1) $=0.48$

${ }^{1} \mathrm{H}$ NMR (400 MHz, CDCl 3 ): $\delta$ 7.36-7.28 (m, 3H, H9 and H10), 7.21-7.19 (m, 2H, H8), 6.80 (d, J = 11.5 $\mathrm{Hz}, 1 \mathrm{H}, \mathbf{H 6}$ ), 5.99-5.84 (m, 2H, H2 and H5), 5.47-5.36 (m, 3H, H1 A, H1 $\mathrm{B}$ and $\mathbf{H 4}), 4.89-4.85(\mathrm{~m}, 1 \mathrm{H}$, H3), 1.47 (br s, 9H, H13), 1.44 (br s, 9H, H16).

${ }^{13} \mathrm{C}$ NMR (100 MHz, CDCl $)$ : $\delta 159.2$ (C11), 158.2 (C14), 135.8 (C7), 135.0 (C6), 132.3 (C2), 128.7 (C8), 128.5 (C9), 127.9 (C10), 126.1 (C5), 120.6 (C1), 82.1 (C12 and C15), 66.8 (C3), 61.9 (C4), 28.3 (C13 or C16), 28.3 (C13 or C16).

FTIR (ATR) v $\left(\mathrm{cm}^{-1}\right): 2978,2932,1702(\mathrm{C}=\mathrm{O})$.

HRMS (ESI): $m / z$ calculated for: $\mathrm{C}_{22} \mathrm{H}_{30} \mathrm{~N}_{2} \mathrm{O}_{4}[\mathrm{M}+\mathrm{Na}]^{+} 409.2098$, found 409.2096; [2M+Na] ${ }^{+}$795.4303, found 795.4299 .

\section{E-isomer 9b}

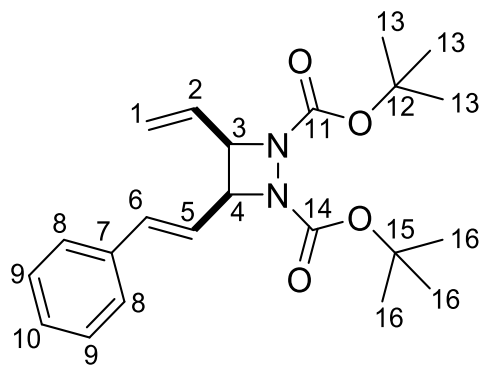

$R_{f}($ Hexane-EtOAc, $2: 1)=0.44$

${ }^{1} \mathrm{H}$ NMR (400 MHz, CDCl 3 ): $\delta$ 7.40-7.25 (m, 5H, H8, H9, H10), 6.65 (d, J = $15.7 \mathrm{~Hz}, 1 \mathrm{H}, \mathbf{H 6}$ ), 6.24 (dd, $J=15.7,8.6 \mathrm{~Hz}, 1 \mathrm{H}, \mathbf{H 5}), 5.97-5.88(\mathrm{~m}, 1 \mathrm{H}, \mathbf{H} 2), 5.45-5.34\left(\mathrm{~m}, 2 \mathrm{H}, \mathbf{H} \mathbf{1}_{\mathrm{A}}\right.$ and $\left.\mathbf{H} \mathbf{1}_{\mathrm{B}}\right), 5.07-5.01(\mathrm{~m}, 1 \mathrm{H}$, H4), 4.97-4.92 (m, 1H, H3), 1.48-4.41 (m, 18H, H13 and H16).

${ }^{13} \mathrm{C} \mathrm{NMR}\left(100 \mathrm{MHz}, \mathrm{CDCl}_{3}\right.$ ): $\delta 158.9$ (C), 158.6 (C), 136.1 (C7), 135.9 (C6), 132.5 (C2), 128.8 (C9), 128.4 (C10), 126.9 (C8), 123.5 (C5), 120.8 (C1), 82.1 (C12 or C15), 81.9 (C12 or C15), 66.8 (C3), 66.5 (C4), 28.3 (C13 or C16), 28.2 (C13 or C16).

FTIR (ATR) v $\left(\mathrm{cm}^{-1}\right)$ : 2980, 2932, $1709(\mathrm{C}=\mathrm{O})$.

HRMS (ESI): $m / z$ calculated for: $\mathrm{C}_{22} \mathrm{H}_{30} \mathrm{~N}_{2} \mathrm{O}_{4}[\mathrm{M}+\mathrm{Na}]^{+} 409.2098$, found 409.2092; [2M+Na] ${ }^{+}$795.4303, found 795.4296 . 


\section{Di-propan-2-yl (1Z,3Z)-buta-1,3-diene-1,4-diylbiscarbamate 11c}<smiles>CC(C)OC(=O)N/C=C/C=C/NC(=O)OC(C)C</smiles>

$S \mathrm{Sl}_{2}$ prepared according to a previously reported procedure. ${ }^{7}$ Note: It was essential for dry THF without stabilisers to be used for the preparation of $\mathrm{Sml}_{2}$. A solution of $\mathrm{Sml}_{2}$ in THF $(0.072 \mathrm{M}, 13.7 \mathrm{~mL}, 0.98$ $\mathrm{mmol}, 2.5 \mathrm{eq}$ ) was added dropwise to a stirred deoxygenated solution of bicyclic 1,2-diazetidine $2 \mathrm{c}$ (100 $\mathrm{mg}, 0.39 \mathrm{mmol}, 1.0 \mathrm{eq})$ in THF $(2 \mathrm{~mL})$ at room temperature under argon, then stirred at room temperature for 30 minutes. The reaction mixture was purged with air until the colour changed from blue to yellow and the solvent was removed under reduced pressure to give the crude product. Purification by flash column chromatography on silica gel (eluent: hexane- $\mathrm{Et}_{2} \mathrm{O}, 1: 1$ ) gave diene 11c (69 $\mathrm{mg}, 0.27 \mathrm{mmol}, 68 \%$ ) as a white solid.

$R_{f}\left(\right.$ Hexane- $\left.\mathrm{Et}_{2} \mathrm{O}, 1: 1\right)=0.35$

$\mathrm{mp}=190-193{ }^{\circ} \mathrm{C}$

${ }^{1} \mathrm{H}$ NMR (400 MHz, CDCl 3 ): $\delta$ 6.60-6.44 (br m, 4H, H1 and H2), 5.29 (br s, 2H, NH), 4.99 (sept, J = 6.2 $\mathrm{Hz}, 2 \mathrm{H}, \mathrm{H} 4), 4.99$ (d, J = $6.2 \mathrm{~Hz}, 12 \mathrm{H}, \mathrm{H5}$ ).

${ }^{13} \mathrm{C} \mathrm{NMR} \mathrm{(100} \mathrm{MHz,} \mathrm{CDCl} 3$ ): $\delta 153.2$ (C3), 122.5 (C1), 100.6 (C2), 69.5 (C4), 22.2 (C5).

FTIR (ATR) v (cm $\left.{ }^{-1}\right): 3278(\mathrm{NH}), 3000,1690(\mathrm{C}=\mathrm{O}), 1623(\mathrm{C}=\mathrm{O})$.

\section{Di-tert-butyl (1Z,3Z)-buta-1,3-diene-1,4-diylbiscarbamate 11d}<smiles>CC(C)(C)OC(=O)N/C=C/C=C/NC(=O)OC(C)(C)S</smiles>

$\mathrm{Sml}_{2}$ prepared according to a previously reported procedure. ${ }^{7}$ Note: It was essential for dry THF without stabilisers to be used for the preparation of $\mathrm{Sml}_{2}$. A solution of $\mathrm{Sml}_{2}$ in THF $(0.075 \mathrm{M}, 21.0 \mathrm{~mL}, 1.57$ $\mathrm{mmol}, 2.2 \mathrm{eq}$ ) was added in one portion to a stirred deoxygenated solution of bicyclic 1,2-diazetidine 2d $(202 \mathrm{mg}, 0.72 \mathrm{mmol}, 1.0 \mathrm{eq})$ in $\mathrm{MeOH}(7.2 \mathrm{~mL})$ at room temperature under argon, then stirred at room temperature for 30 minutes. The reaction mixture was purged with air until the colour changed from blue to yellow and the solvent was removed under reduced pressure to give the crude product. Purification by flash column chromatography on silica gel (eluent: hexane-EtOAc, 14:1 $\rightarrow 9: 1$ ) gave diene 11d (48 mg, $0.17 \mathrm{mmol}, 24 \%$ ) as a white solid.

$R_{f}($ Hexane-EtOAc, 2:1) $=0.48$

$\mathrm{mp}=206-208^{\circ} \mathrm{C}$ (decomposition)

${ }^{1} \mathrm{H}$ NMR (400 MHz, $d_{6}$-DMSO): $\delta$ 9.07-8.77 (br m, 2H, NH), 6.15-6.08 (br m, 2H, H1), 5.65-5.54 (br m, 2H, H2), 1.43 (br s, 18H, H5).

${ }^{13} \mathrm{C}$ NMR (100 MHz, d6-DMSO): $\delta 153.1$ (C3), 121.0 (C1), 102.2 (C2), 79.2 (C4), 28.0 (C5). 
FTIR (ATR) v $\left(\mathrm{cm}^{-1}\right): 3325(\mathrm{NH}), 2980,2935,1690(\mathrm{C}=\mathrm{O}), 1621(\mathrm{C}=\mathrm{O})$.

HRMS (ESI): $m / z$ calculated for: $\mathrm{C}_{14} \mathrm{H}_{24} \mathrm{~N}_{2} \mathrm{O}_{4}[\mathrm{M}+\mathrm{Na}]^{+} 307.1628$, found 307.1628.

\section{Di-tert-butyl 1-(cyclobut-2-en-1-yl)hydrazine-1,2-dicarboxylate 12d}

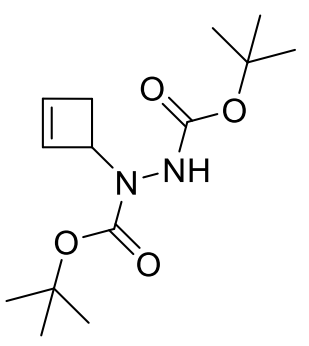

A solution of bicyclic 1,2-diazetidine $\mathbf{2} \mathbf{d}(50 \mathrm{mg}, 0.18 \mathrm{mmol}, 1.0 \mathrm{eq})$ in THF $(3 \mathrm{~mL})$ was added to liquid ammonia $(3 \mathrm{~mL})$ at $-78{ }^{\circ} \mathrm{C}$ under argon. Sodium metal $(41 \mathrm{mg}, 1.77 \mathrm{mmol}, 10 \mathrm{eq})$ was added portion wise at $-78{ }^{\circ} \mathrm{C}$, then the reaction mixture was warmed to room temperature. The reaction mixture was left at room temperature overnight to remove the ammonia. The residue was dissolved in EtOAc $(5 \mathrm{~mL})$, dried $\left(\mathrm{MgSO}_{4}\right)$ and the solvent removed under reduced pressure to give the crude product. Purification by flash column chromatography on silica gel (eluent: hexane-EtOAc, 9:1 $\rightarrow 4: 1$ ) gave a mixture of cyclobutene $12 \mathrm{~d}$ and diene $\mathbf{1 3 d}$ (44 $\mathrm{mg}, 0.16 \mathrm{mmol}, 87 \%, 2.4: 1.0 \mathbf{1 2 d : 1 3 d}$ ) as a white cloudy oil.

Note: Diene 13d can be isolated as a white solid with careful purification. The purification of cyclobutene $\mathbf{1 2 d}$ always resulted in the presence of diene $13 d$.

\section{Cyclobutene 12d}

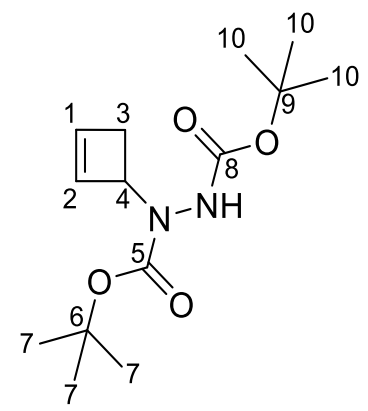

$R_{f}($ Hexane-EtOAc, 2:1) $=0.46$

${ }^{1} \mathrm{H}$ NMR (400 MHz, $\left.d_{6}-\mathrm{DMSO}\right): \delta$ 8.92-8.44 (br m, 1H, NH), 6.14-6.09 (br m, 1H, H1), 5.92-5.83 (br m, 1H, H2), 5.06-4.86 (br m, 1H, H4), 2.69-2.40 (br m, 2H, H3 ${ }_{\mathrm{A}}$ and $\mathbf{H} 3_{\mathrm{B}}$ ), 1.43-1.35 (br m, 18H, $\mathbf{H} 7$ and H10).

${ }^{13} \mathrm{C}$ NMR (100 MHz, $d_{6}$-DMSO, additional peaks due to rotamers annotated by an asterisk): $\delta 155.6^{*}$ (C5 or C8), 155.4 (C5 or C8), 154.0* (C5 or C8), 153.8 (C5 or C8), 137.2 (C1), 136.4* (C2), 136.2 (C2), 80.0* (C6 or C9), 79.6 (C6 or C9), 79.1 (C6 or C9), 56.3* (C4), 55.9 (C4), 55.6* (C4), 36.3 (C3), 28.0 (C7 or C10), 27.9 (C7 or C10).

FTIR (ATR) v $\left(\mathrm{cm}^{-1}\right): 3301(\mathrm{NH}), 2976,2928,1702(\mathrm{C}=\mathrm{O})$.

HRMS (ESI): $m / z$ calculated for: $\mathrm{C}_{14} \mathrm{H}_{24} \mathrm{~N}_{2} \mathrm{O}_{4}[\mathrm{M}+\mathrm{Na}]^{+} 307.1628$, found 307.1637 .

Important NOE contacts: 


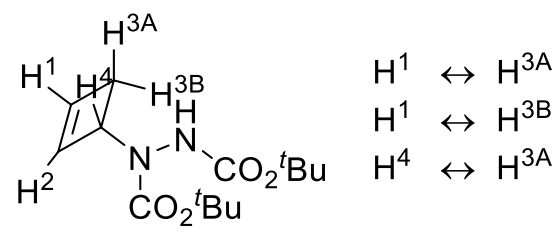

Diene 13d

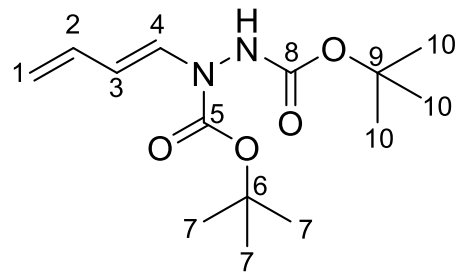

$R_{f}($ Hexane-EtOAc, $2: 1)=0.52$

$\mathrm{mp}=96-98^{\circ} \mathrm{C}$

${ }^{1} \mathrm{H}$ NMR (400 MHz, $348 \mathrm{~K}, d_{6}$-DMSO): $\delta$ 9.24-8.8.69 (br m, 1H, NH), 7.06 (br d, J = 13.6 Hz, 1H, H4), 6.41-6.32 (m, 1H, H2), 5.68-5.62 (m, 1H, H3), 5.06-5.01 (m, 1H, H1 $\left.{ }_{A}\right)$, 4.89-4.86 (m, H, H1 B $), 1.47-1.41$ (br m, 18H, $\mathbf{H 7}$ and $\mathbf{H 1 0}$ ).

${ }^{13} \mathrm{C}$ NMR (100 MHz, $348 \mathrm{~K}, d_{6}$-DMSO): $\delta 153.7$ (C5 and C8), 134.3 (C2), 130.2 (C4), 112.7 (C1), 109.6 (C2), 81.4 (C6 or C9), 79.5 (C6 or C9), 27.7 (C7 or C10), 27.4 (C7 or C10).

FTIR (ATR) v $\left(\mathrm{cm}^{-1}\right): 3289(\mathrm{NH}), 2978,2932,1715(\mathrm{C}=\mathrm{O}), 1649(\mathrm{C}=\mathrm{O})$.

HRMS (ESI or APCI): target mass not found.

\section{Di-tert-butyl 2,3-diazabicyclo[2.2.0]hexane-2,3-dicarboxylate 14d}

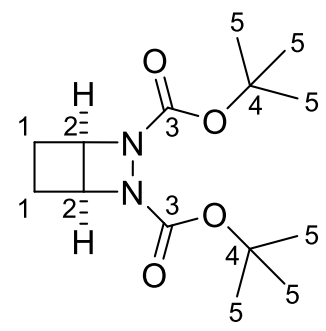

Hydrazine hydrate $(2.6 \mathrm{~mL}, 53.1 \mathrm{mmol}, 66 \mathrm{eq})$ was added in one portion to a solution of bicyclic 1,2diazetidine $2 \mathrm{~d}$ ( $250 \mathrm{mg}, 0.88 \mathrm{mmol}, 1.0 \mathrm{eq})$ in $\mathrm{EtOH}(2 \mathrm{~mL})$. The resulting solution was cooled to $0{ }^{\circ} \mathrm{C}$, then $\mathrm{H}_{2} \mathrm{O}_{2}$ (295 $\mu \mathrm{L}$ of a $50 \%$ w/v aqueous solution, $\left.17.7 \mathrm{mmol}, 20 \mathrm{eq}\right)$. was added dropwise over 20 mins at $0{ }^{\circ} \mathrm{C}$. The resulting solution was stirred at $0{ }^{\circ} \mathrm{C}$ for one hour, then water $(20 \mathrm{~mL})$ was added. The mixture was extracted with EtOAc $(3 \times 10 \mathrm{~mL})$, dried $\left(\mathrm{MgSO}_{4}\right)$ and the solvent was removed under reduced pressure to give the crude product. Purification by flash column chromatography on silica gel (eluent: hexane-EtOAc, 4:1) gave bicycle $14 \mathrm{~d}$ (250 mg, $0.88 \mathrm{mmol}, 99 \%$ ) as a colourless oil.

$R_{f}($ Hexane-EtOAc, 4:1) $=0.31$

${ }^{1} \mathrm{H}$ NMR (400 MHz, CDCl 3 ): $\delta$ 4.68-4.66 (br m, 2H, H2), 2.55-2.49 (br m, 4H, H1), 1.49 (br s, 18H, H5). ${ }^{13} \mathrm{C} \mathrm{NMR}$ (100 MHz, CDCl $)$ : $\delta 158.7$ (C3), 81.9 (C4), 64.1 (C2), 28.3 (C5), 27.4 (C1). FTIR $(A T R) \vee\left(\mathrm{cm}^{-1}\right): 2980,2945,1737(\mathrm{C}=\mathrm{O}), 1711(\mathrm{C}=\mathrm{O})$. 
HRMS (ESI): $m / z$ calculated for: $\mathrm{C}_{14} \mathrm{H}_{24} \mathrm{~N}_{2} \mathrm{O}_{4}[\mathrm{M}+\mathrm{Na}]^{+} 307.1628$, found 307.1617.

tert-Butyl methyl 2,3-diazabicyclo[2.2.0]hexane-2,3-dicarboxylate $14 \mathrm{~g}$

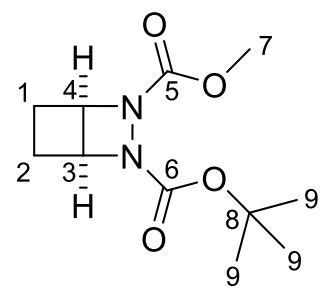

A solution of acetic acid $(715 \mu \mathrm{L}, 12.5 \mathrm{mmol}, 30 \mathrm{eq})$ in $\mathrm{CH}_{2} \mathrm{Cl}_{2}(5 \mathrm{~mL})$ was added dropwise over 1 hour at $0{ }^{\circ} \mathrm{C}$ to a stirred suspension of dipotassium azodicarboxylate $(1.21 \mathrm{~g}, 6.24 \mathrm{mmol}, 15 \mathrm{eq})$ and bicyclic 1,2-diazetidine $\mathbf{2 g}$ (100 mg, $0.42 \mathrm{mmol}, 1.0 \mathrm{eq})$ in $\mathrm{CH}_{2} \mathrm{Cl}_{2}(5 \mathrm{~mL})$. The resulting suspension was stirred and allowed to warm gradually to room temperature over 12 hours, then filtered and washed with $\mathrm{CH}_{2} \mathrm{Cl}_{2}$ $(5 \times 5 \mathrm{~mL})$. The filtrate was evaporated under reduced pressure to give the crude product. Purification by flash column chromatography on silica gel (eluent: hexane-EtOAc, 3:1) gave bicycle $\mathbf{1 4 g}$ (99 $\mathrm{mg}$, $0.41 \mathrm{mmol}, 98 \%$ ) as a colourless oil.

$R_{f}($ Hexane-EtOAc, 3:1) $=0.23$

${ }^{1} \mathrm{H}$ NMR (400 MHz, $\left.\mathrm{CDCl}_{3}\right): \delta 4.77-4.73(\mathrm{~m}, 1 \mathrm{H}, \mathbf{H} 3$ or $\mathbf{H 4}), 4.71-4.68(\mathrm{~m}, 1 \mathrm{H}, \mathbf{H} 3$ or $\mathbf{H 4}), 3.79(\mathrm{~s}, 3 \mathrm{H}$, H7), 2.58-2.50 (m, 4H, H1 and H2), 1.49 (br s, 9H, H9).

${ }^{13} \mathrm{C} \mathrm{NMR} \mathrm{(100} \mathrm{MHz,} \mathrm{CDCl}_{3}$ ): $\delta 160.2$ and 158.6 (C5 and C6), 82.3 (C8), 64.53 and 64.49 (C3 and C4), 53.4 (C7), 28.3 (C9), 27.40 and 27.38 (C1 and C2).

FTIR (ATR) v $\left(\mathrm{cm}^{-1}\right): 2952,2980,1702(\mathrm{C}=\mathrm{O})$.

HRMS (ESI): $m / z$ calculated for: $\mathrm{C}_{11} \mathrm{H}_{18} \mathrm{~N}_{2} \mathrm{O}_{4}[\mathrm{M}+\mathrm{Na}]^{+} 265.1159$, found 265.1151 .

\section{Di-tert-butyl (1R,2S)-cyclobutane-1,2-diylbiscarbamate 15d}

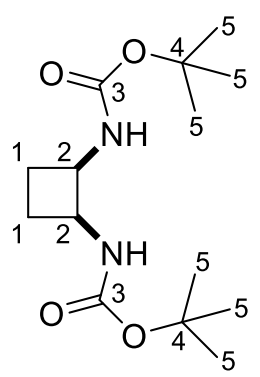

A solution of bicyclic 1,2-diazetidine $14 \mathrm{~d}(136 \mathrm{mg}, 0.48 \mathrm{mmol}, 1.0 \mathrm{eq})$ in THF ( $5 \mathrm{~mL}$ ) was added to liquid ammonia $(5 \mathrm{~mL})$ at $-78{ }^{\circ} \mathrm{C}$ under argon. Sodium metal $(110 \mathrm{mg}, 4.78 \mathrm{mmol}, 10 \mathrm{eq})$ was added portion wise at $-78{ }^{\circ} \mathrm{C}$, then the reaction mixture was stirred at $-78^{\circ} \mathrm{C}$ for 15 mins. Solid $\mathrm{NH}_{4} \mathrm{Cl}(100 \mathrm{mg})$ was added at $-78^{\circ} \mathrm{C}$, and the mixture was allowed to warm slowly to room temperature. The reaction mixture was left at room temperature overnight to allow complete evaporation of the ammonia. The residue was 
dissolved in $\mathrm{CH}_{2} \mathrm{Cl}_{2}(5 \mathrm{~mL})$ and filtered, then washed with further $\mathrm{CH}_{2} \mathrm{Cl}_{2}(4 \times 5 \mathrm{~mL})$. The solvent was removed under reduced pressure to give the crude product. Purification by flash column chromatography on silica gel (eluent: hexane-EtOAc, 3:1) gave diamine $15 \mathrm{~d}(111 \mathrm{mg}, 0.39 \mathrm{mmol}, 81 \%)$ as a white solid.

$\mathrm{mp}=165-168^{\circ} \mathrm{C}$

$R_{f}($ Hexane-EtOAc, 3:1) $=0.30$

${ }^{1} \mathrm{H}$ NMR (400 MHz, $d_{6}$-DMSO; rotamers present): $\delta$ 7.16-7.10, 6.84-6.66 and 6.33-6.26 (all br m, 2H, $\mathbf{N H}$ ), 4.14-3.92 and 3.86-3.65 (both br m, 2H, H2), 2.07-1.92 and 1.87-1.66 (both br m, 2H, $3 \times \mathbf{H 1}$ ), 1.52-1.19 (br m, 19H, $\mathbf{H} 5$ and $3 \times \mathbf{H 1})$.

${ }^{13} \mathrm{C}$ NMR (100 MHz, $d_{6}$-DMSO; rotamers present): $\delta 155.1$ and 154.9 (C3), 79.2 and 77.8 (C4), 52.2 and 49.2 (C2), 28.2 (C5), 25.0 and 22.7 (C1).

FTIR (ATR) v $\left(\mathrm{cm}^{-1}\right): 3330(\mathrm{NH}), 2976,1681(\mathrm{C}=\mathrm{O})$.

HRMS (ESI): $m / z$ calculated for: $\mathrm{C}_{14} \mathrm{H}_{26} \mathrm{~N}_{2} \mathrm{O}_{4}[\mathrm{M}+\mathrm{Na}]^{+} 309.1785$, found 309.1780 .

\section{tert-Butyl methyl cis-cyclobutane-1,2-diylbiscarbamate 15g}

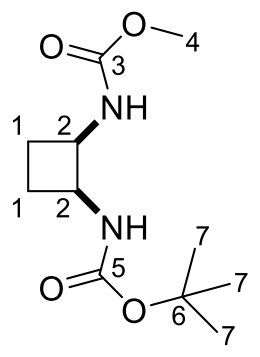

A solution of bicyclic 1,2-diazetidine $14 \mathrm{~g}(87 \mathrm{mg}, 0.36 \mathrm{mmol}, 1.0 \mathrm{eq})$ in THF (3 mL) was added to liquid ammonia $(5 \mathrm{~mL})$ at $-78{ }^{\circ} \mathrm{C}$ under argon. Sodium metal ( $\left.83 \mathrm{mg}, 3.59 \mathrm{mmol}, 10 \mathrm{eq}\right)$ was added portion wise at $-78{ }^{\circ} \mathrm{C}$, then the reaction mixture was stirred at $-78^{\circ} \mathrm{C}$ for 15 mins. Solid $\mathrm{NH}_{4} \mathrm{Cl}(100 \mathrm{mg})$ was added at $-78^{\circ} \mathrm{C}$, and the mixture was allowed to warm slowly to room temperature. The reaction mixture was left at room temperature overnight to allow complete evaporation of the ammonia. The residue was dissolved in $\mathrm{CH}_{2} \mathrm{Cl}_{2}(5 \mathrm{~mL})$, filtered, washed with further $\mathrm{CH}_{2} \mathrm{Cl}_{2}(4 \times 5 \mathrm{~mL})$ and the solvent was removed under reduced pressure to give the crude product. Purification by flash column chromatography on silica gel (eluent: hexane-EtOAc, 2:1) gave diamine $15 \mathrm{~g}(67 \mathrm{mg}, 0.27 \mathrm{mmol}, 76 \%)$ as a white solid.

$\mathrm{mp}=124-126{ }^{\circ} \mathrm{C}$

$R_{f}($ Hexane-EtOAc, 2:1) $=0.20$

${ }^{1} \mathrm{H}$ NMR (400 MHz, $d_{6}$-DMSO;5:4:2:1 mixture of rotamers); $\delta 7.43$ (br d, $J=7.2 \mathrm{~Hz}, 0.7 \mathrm{H}, \mathbf{N H}$ ), 7.12 (br $\mathrm{d}, J=7.8 \mathrm{~Hz}, 0.8 \mathrm{H}, \mathbf{N H}), 7.01(\mathrm{br} \mathrm{d}, J=8.1 \mathrm{~Hz}, 0.2 \mathrm{H}, \mathbf{N H}$ ), $6.72(\mathrm{br} \mathrm{d}, J=7.4 \mathrm{~Hz}, 0.3 \mathrm{H}, \mathbf{N H}), 4.19-3.67$ (br m, 2H, H3 and H4), 3.51 and 3.49 (s, 3H, H6), 2.07-1.94 and 1.90-1.68 (br m, 2.5H, $\mathbf{H 1}$ and $\mathbf{H 2}$ ), 1.51-1.20 (br m, 10.5H, $\mathbf{H} \mathbf{9}$ and $\mathbf{H} 1$ and/or $\mathbf{H} 2$ ).

${ }^{13} \mathrm{C} \mathrm{NMR}$ (100 MHz, $\mathrm{CDCl}_{3}$; rotamers present); $\delta$ 155.8, 155.6, 154.7 and 154.5 (C5 and C7), 77.9 and 77.6 (C8), 52.6, 52.0, 51.1, 51.3, 49.7 and 49.2 (C6, C3 and C4), 28.22 and 28.16 (C9), 25.1, 22.8 and 22.6 (C1 and $\mathbf{C 2}$ ).

FTIR (ATR) v $\left(\mathrm{cm}^{-1}\right): 3319(\mathrm{NH}), 2978,2950,1696(\mathrm{C}=\mathrm{O}), 1683(\mathrm{C}=\mathrm{O})$. 
HRMS (ESI): $m / z$ calculated for: $\mathrm{C}_{11} \mathrm{H}_{20} \mathrm{~N}_{2} \mathrm{O}_{4}[\mathrm{M}+\mathrm{Na}]^{+} 267.1315$, found 267.1314.

tert-Butyl 4-oxo-5-oxa-2,3-diazabicyclo[4.2.0]oct-7-ene-2-carboxylate 16<smiles>CC(C)(C)OC(=O)N1NC(=O)O[C@H]2C=C[C@@H]21</smiles>

A solution of bicyclic 1,2-diazetidine $\mathbf{2 d}(512 \mathrm{mg}, 1.81 \mathrm{mmol})$ in 1,4-dioxane $(3.5 \mathrm{~mL})$ was heated at reflux for 24 hours. The reaction mixture was cooled to room temperature, then a $1 \mathrm{M}$ aqueous solution of $\mathrm{HCl}(1 \mathrm{~mL})$ was added and the mixture stirred for at room temperature for 1 hour. The reaction mixture was quenched with a saturated aqueous solution of $\mathrm{NaHCO}_{3}(1 \mathrm{~mL})$, the organic layer was separated and the aqueous layer was extracted with $\mathrm{CH}_{2} \mathrm{Cl}_{2}(5 \times 5 \mathrm{~mL})$. The combined organic layers were dried $\left(\mathrm{MgSO}_{4}\right)$ and the solvent was evaporated under reduced pressure to give the crude product. Purification by flash column chromatography on silica gel (eluent: hexane-EtOAc, 2:1) gave bicycle 16 (366 mg, $1.62 \mathrm{mmol}, 89 \%)$ as a white solid.

$R_{f}($ Hexane-EtOAc, 2:1) $=0.07$

$\mathrm{mp}=134-136{ }^{\circ} \mathrm{C}$

${ }^{1} \mathrm{H} \mathrm{NMR}\left(400 \mathrm{MHz}, \mathrm{CDCl}_{3}\right) ; \delta 7.21$ (br s, 1H, NH), 6.42-6.41 (br m, 1H, H1 or H2), 6.34-6.33 (br m, 1H, H1 or H2), 5.44-5.42 (br m, 2H, H3 and H4), 1.50 (s, 9H, H8).

${ }^{13} \mathrm{C}$ NMR (100 MHz, CDCl 3 ); $\delta 153.6$ (C5 or C6), 151.9 (C5 or C6), 141.5 (C1 or C2), 140.2 (C1 or C2), 83.6 (C7), 81.5 (C3), 60.2 (C4), 28.3 (C8).

FTIR (ATR) v (cm-1): $3293(\mathrm{NH}), 2982,2933,1728(\mathrm{C}=\mathrm{O}), 1683(\mathrm{C}=\mathrm{O})$.

HRMS (ESI): $\mathrm{m} / \mathrm{z}$ calculated for: $\mathrm{C}_{10} \mathrm{H}_{14} \mathrm{~N}_{2} \mathrm{O}_{4}[\mathrm{M}+\mathrm{Na}]^{+} 249.0846$, found 249.0839 .

rac-Di-tert-butyl (1S,6R)-4-oxo-5-oxa-2,3-diazabicyclo[4.2.0]octane-2,3-dicarboxylate 17

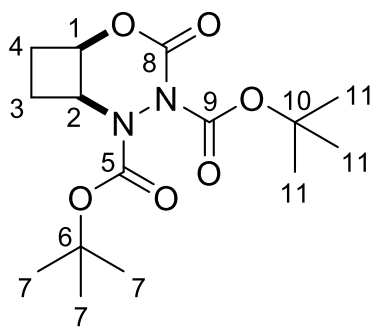

A solution of acetic acid $(2.5 \mathrm{~mL}, 44.2 \mathrm{mmol}, 100 \mathrm{eq})$ in $\mathrm{CH}_{2} \mathrm{Cl}_{2}(5 \mathrm{~mL})$ was added dropwise over 1 hour at $0{ }^{\circ} \mathrm{C}$ to a stirred suspension of dipotassium azodicarboxylate $(4.29 \mathrm{~g}, 22.1 \mathrm{mmol}, 50 \mathrm{eq})$ and bicyclic 1,2-diazetidine $\mathbf{2 g}$ (100 mg, $0.44 \mathrm{mmol}, 1.0 \mathrm{eq})$ in $\mathrm{CH}_{2} \mathrm{Cl}_{2}(10 \mathrm{~mL})$. The resulting suspension was stirred and allowed to warm gradually to room temperature over 12 hours, then filtered and washed with $\mathrm{CH}_{2} \mathrm{Cl}_{2}$ 
$(5 \times 5 \mathrm{~mL})$. The filtrate was evaporated under reduced pressure to give the crude product, which consisted of starting material and product $(\sim 1: 1)$. The residue was re-subjected twice to the reduction conditions, leading to complete conversion to the cyclobutane, then dissolved in THF $(5 \mathrm{~mL})$. Triethylamine (123 $\mu \mathrm{L}, 0.88 \mathrm{mmol}, 2.0 \mathrm{eq})$ and DMAP ( $5 \mathrm{mg}, 0.04 \mathrm{mmol}, 0.1 \mathrm{eq})$ were added, and the resulting solution was stirred at room temperature for $12 \mathrm{~h}$, then evaporated under reduced pressure. Purification by flash column chromatography on silica gel (eluent: hexane-EtOAc, 4:1) gave bicycle 17 (118 $\mathrm{mg}, 0.36 \mathrm{mmol}, 81 \%$ ) as a white solid.

$R_{f}($ Hexane-EtOAc, 4:1) $=0.20$

$\mathrm{mp}=126-128^{\circ} \mathrm{C}$

${ }^{1} \mathrm{H}$ NMR (400 MHz, CDCl 3 ); $\delta$ 5.18-5.08 (br m, 1H, H4), 5.03 (dt, J = 8.0 Hz, 7.6 Hz, 1H, H1), 2.56-2.41 (m, 2H, H2 and/or H3), 2.32-2.13 (m, 2H, H2 and/or H3), 1.58 (s, 9H, H7 or H11), 1.49 (s, 9H, H7 or H11).

${ }^{13} \mathrm{C}$ NMR (100 MHz, CDCl 3 ); $\delta$ 153.5, 149.7 and 148.4 (C5, C8 and C9 ), 84.7 and 83.6 (C1, C6 and C10), 55.0 (C4), 28.7 (C2 or C3), 28.11 and 28.09 (C7 and C11), 21.8 (C2 or C3).

FTIR (ATR) v $\left(\mathrm{cm}^{-1}\right):$ 2980, $1804(\mathrm{C}=\mathrm{O}), 1776(\mathrm{C}=\mathrm{O}), 1722(\mathrm{C}=\mathrm{O})$.

HRMS (ESI): $m / z$ calculated for: $\mathrm{C}_{15} \mathrm{H}_{24} \mathrm{~N}_{2} \mathrm{O}_{6}[\mathrm{M}+\mathrm{Na}]^{+} 351.1527$, found 351.1524.

rac-Di-tert-butyl 1-[(1S,2R)-2-hydroxycyclobutyl]hydrazine-1,2-dicarboxylate 18

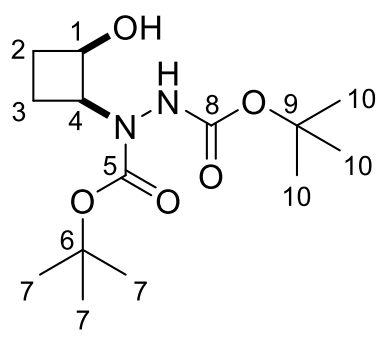

$\mathrm{LiOH} \cdot \mathrm{H}_{2} \mathrm{O}$ (76 mg, $\left.1.81 \mathrm{mmol}, 10 \mathrm{eq}\right)$ was added in one portion to a stirred solution of bicycle 17 (60 $\mathrm{mg}, 0.18 \mathrm{mmol}, 1 \mathrm{eq})$ in THF $(3 \mathrm{~mL})$ and water $(1.5 \mathrm{~mL})$. The resulting mixture was stirred for $14 \mathrm{~h}$ at room temperature, then evaporated under reduced pressure. The residue was dissolved in $\mathrm{CH}_{2} \mathrm{Cl}_{2}(5$ $\mathrm{mL}$ ), filtered, washed with further $\mathrm{CH}_{2} \mathrm{Cl}_{2}(4 \times 5 \mathrm{~mL})$ and the solvent was removed under reduced pressure to give the crude product. Purification by flash column chromatography on silica gel (eluent: hexane-EtOAc, 2:1) gave cyclobutanol $18(41 \mathrm{mg}, 0.14 \mathrm{mmol}, 74 \%)$ as a white solid.

$R_{f}($ Hexane-EtOAc, 2:1) $=0.29$

$\mathrm{mp}=111-113^{\circ} \mathrm{C}$

The ${ }^{1} \mathrm{H}$ and ${ }^{13} \mathrm{C}$ NMR spectra of cyclobutanol 18 are very complex, due to the presence of a number of rotamers. Attempts to simplify the spectra by running samples in different solvents and by using variable-temperature NMR (up to $348 \mathrm{~K}$ ) were unsuccessful, and no improvement was observed.

${ }^{1} \mathrm{H} \mathrm{NMR}$ (400 MHz, d6-DMSO; complex mixture of rotamers): $\delta$ 9.39, 9.26, 8.40 and 8.02 (br s, $1 \mathrm{H}, \mathrm{OH}$ ), 5.33-3.92 (br m, 3H, H1, H4 and NH), 2.16-1.81 (br m, 3H, H2 and H3), 1.73-0.71 (br m, 21H, H2 or H3 plus $\mathbf{H 7}$ and $\mathbf{H 1 0}$ ), 1.49 (br s, 18H, H5). 
${ }^{13} \mathrm{C}$ NMR (100 MHz, $d_{6}$-DMSO; complex mixture of rotamers); $\delta 157.8,155.3,154.7$ and 154.0 (C5 and C8), 80.9, 80.2, 80.0, 79.6, 79.3 and 79.2 (C1, C6 and C9), 69.7, 69.3, 67.9, 67.8, 58.3 (C4), 31.3, 30.8, 29.0, 28.7, 28.2 (C2 and C3), 28.0 and 27.9 (C7 and C10), 22.1, 20.2, 20.1, 17.9, 14.0 (C2 and C3). FTIR (ATR) v ( $\left.\mathrm{cm}^{-1}\right): 3384(\mathrm{OH}), 3299(\mathrm{NH}), 2976,2928,1701(\mathrm{C}=\mathrm{O})$.

HRMS (ESI): $m / z$ calculated for: $\mathrm{C}_{14} \mathrm{H}_{26} \mathrm{~N}_{2} \mathrm{O}_{5}[\mathrm{M}+\mathrm{Na}]^{+} 325.1734$, found 325.1719 .

\section{References}

(1) Dolomanov, O. V.; Bourhis, L. J.; Gildea, R. J.; Howard, J. A. K.; Puschmann, H. OLEX2: A Complete Structure Solution, Refinement and Analysis Program. J. Appl. Crystallogr. 2009, 42, 339-341.

(2) Sheldrick, G. M. A Short History of SHELX. Acta Crystallogr. Sect. A Found. Crystallogr. 2008, $64,112-122$.

(3) Sheldrick, G. M. SHELXT - Integrated Space-Group and Crystal-Structure Determination. Acta Crystallogr. Sect. A Found. Crystallogr. 2015, 71, 3-8.

(4) Altman, L. J.; Semmelhack, M. F.; Hornby, R. B.; Vederas, J. C. Photochemical Isomerisation of Dimethyl 1, 2-Dihydropyridazine-1, 2-Dicarboxylate. Chem. Commun. 1968, 686-687.

(5) Warrener, R. N.; Nunn, E. E.; Paddon-Row, M. N. The Synthesis and Properties of Dimethyl 1,2-Diazetine-1,2dicarboxylate, a Potentially Aromatic Molecule. Aust. J. Chem. 1979, 32, 2659-2674.

(6) Stearns, R.; Ortiz de Montellano, P. R. Inactivation of Cytochrome P-450 by a Catalytically Generated Cyclobutadiene Species. J. Am. Chem. Soc. 1985, 107, 234-240.

(7) Szostak, M.; Spain, M.; Procter, D. J. Preparation of Samarium(II) lodide: Quantitative Evaluation of the Effect of Water, Oxygen, and Peroxide Content, Preparative Methods, and the Activation of Samarium Metal. J. Org. Chem. 2012, 77, 3049-3059. 


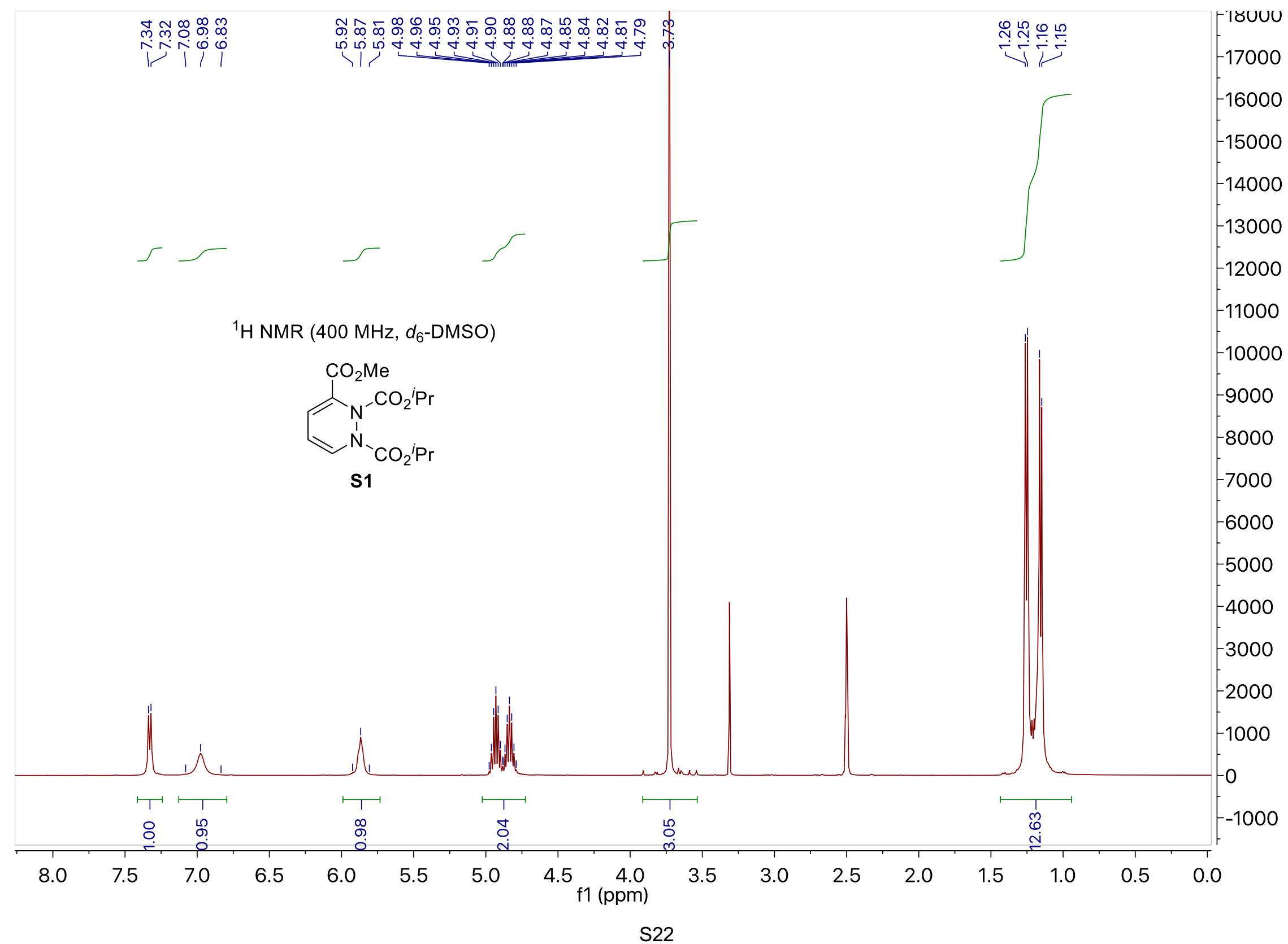




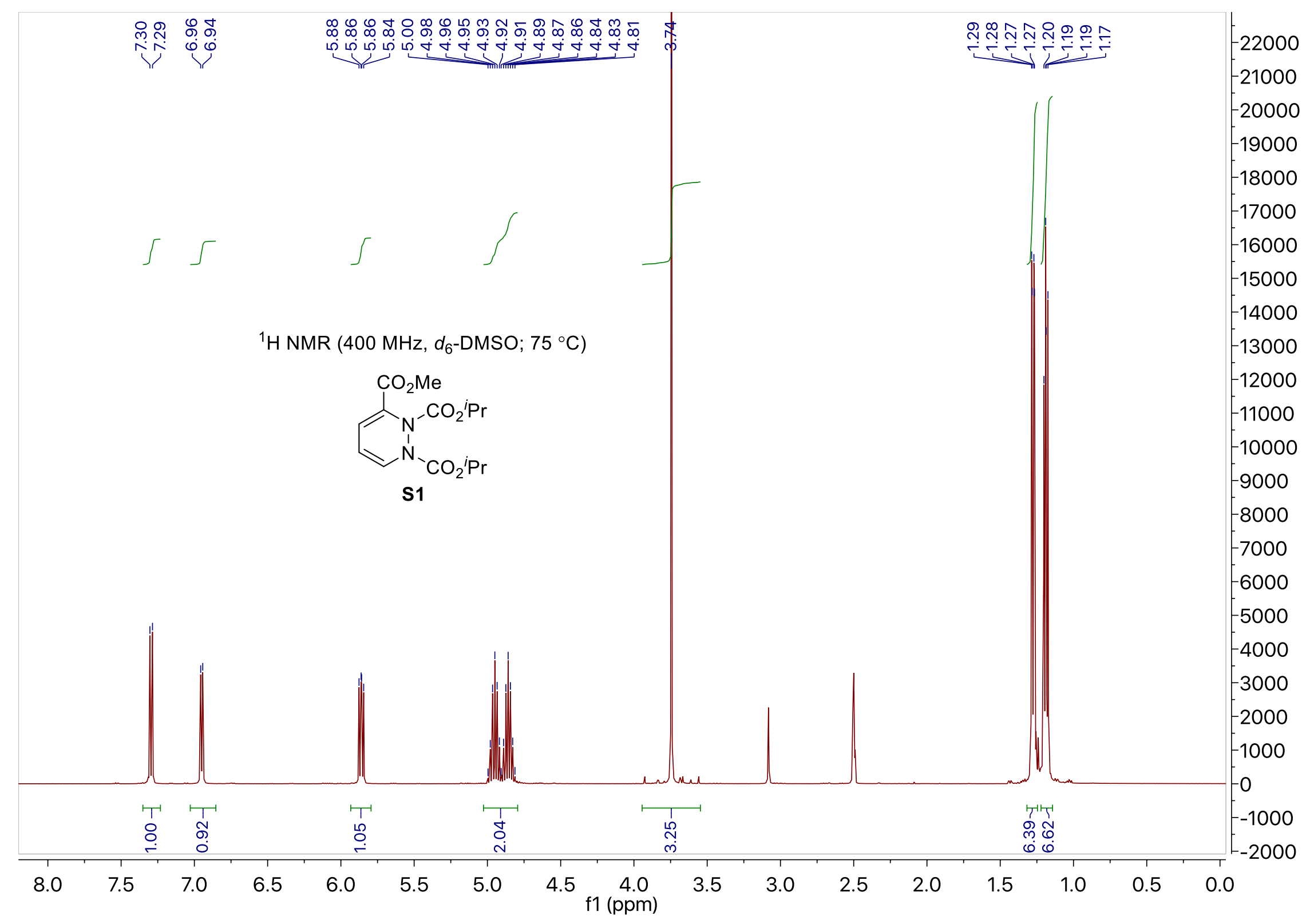




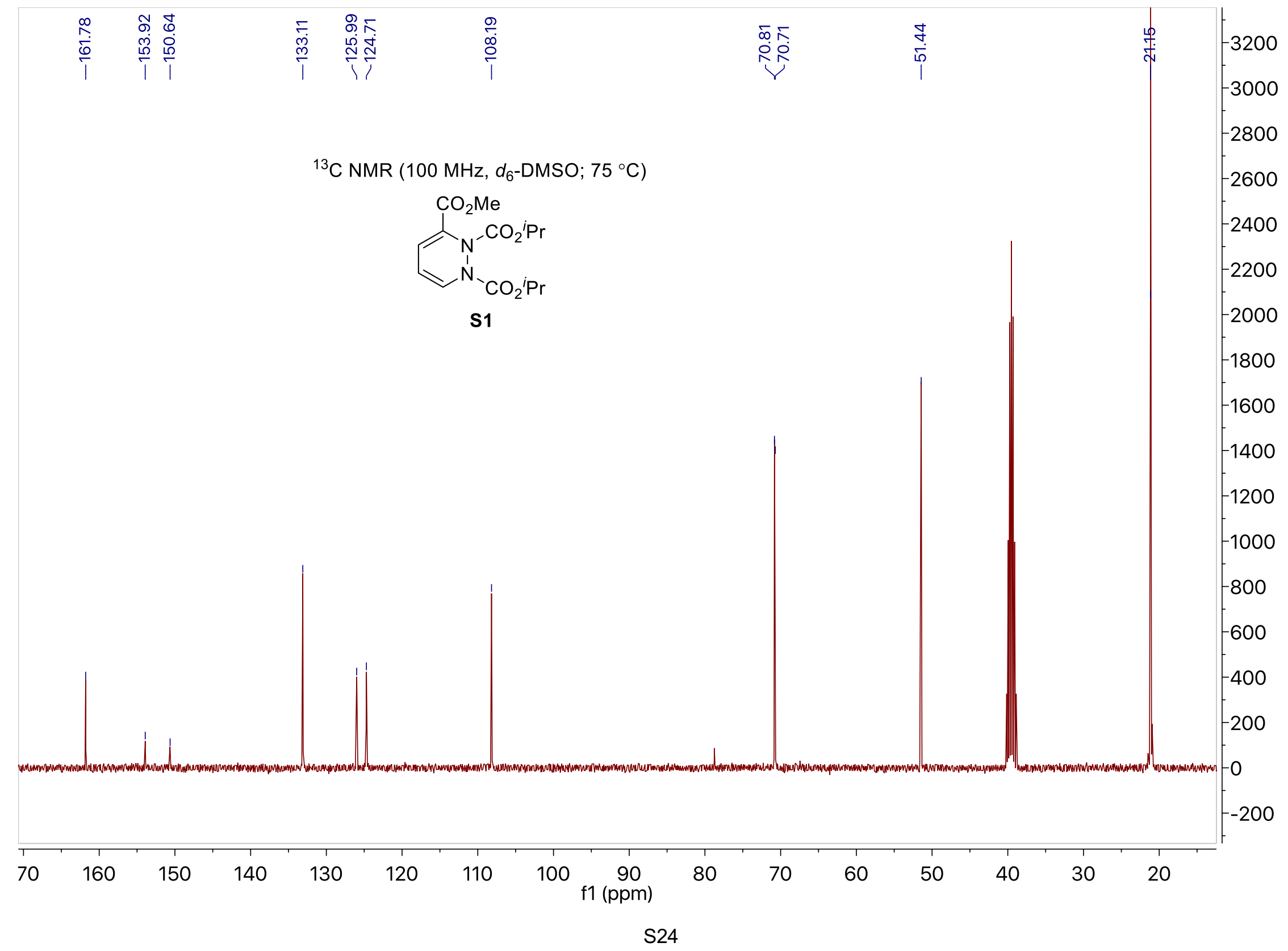




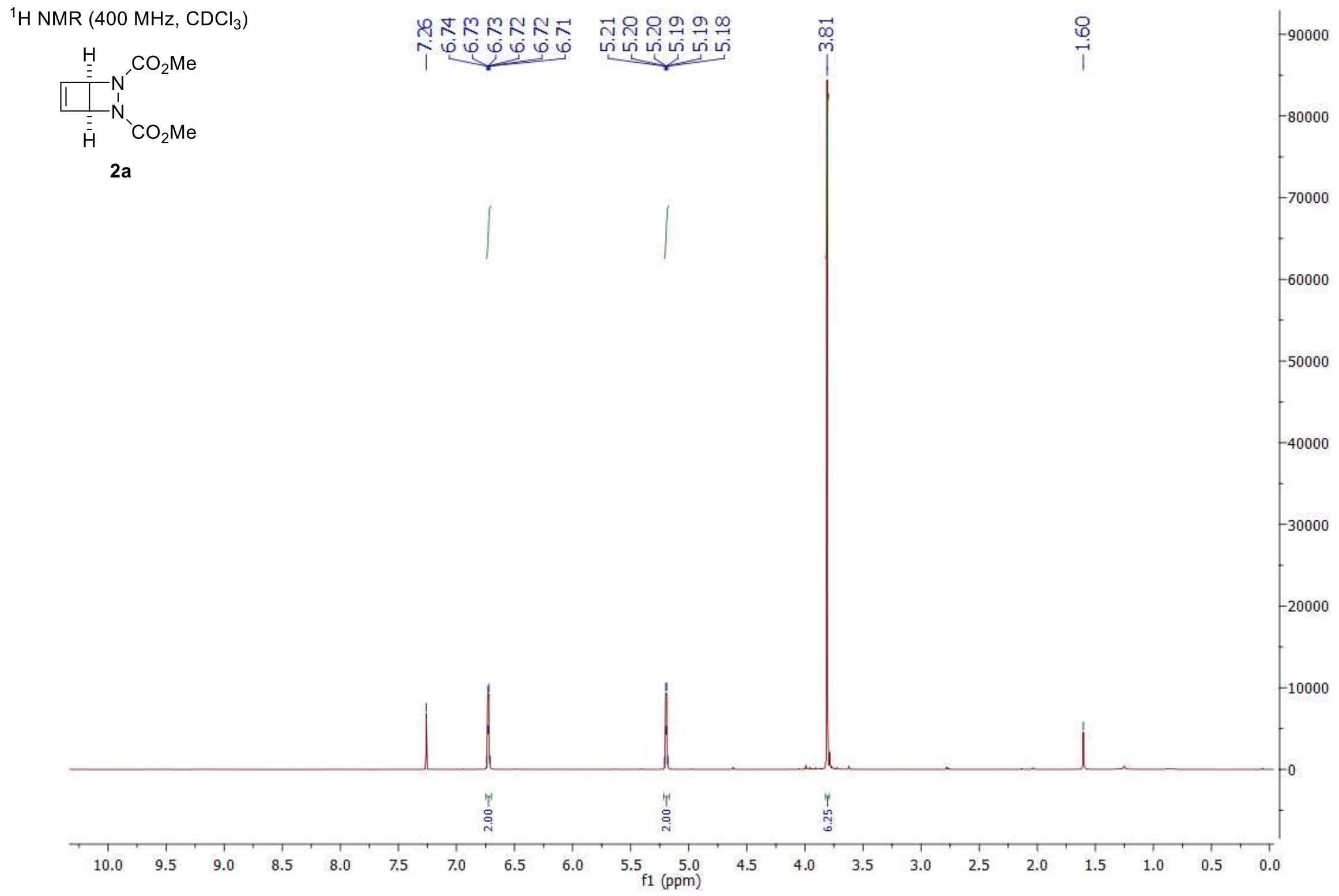




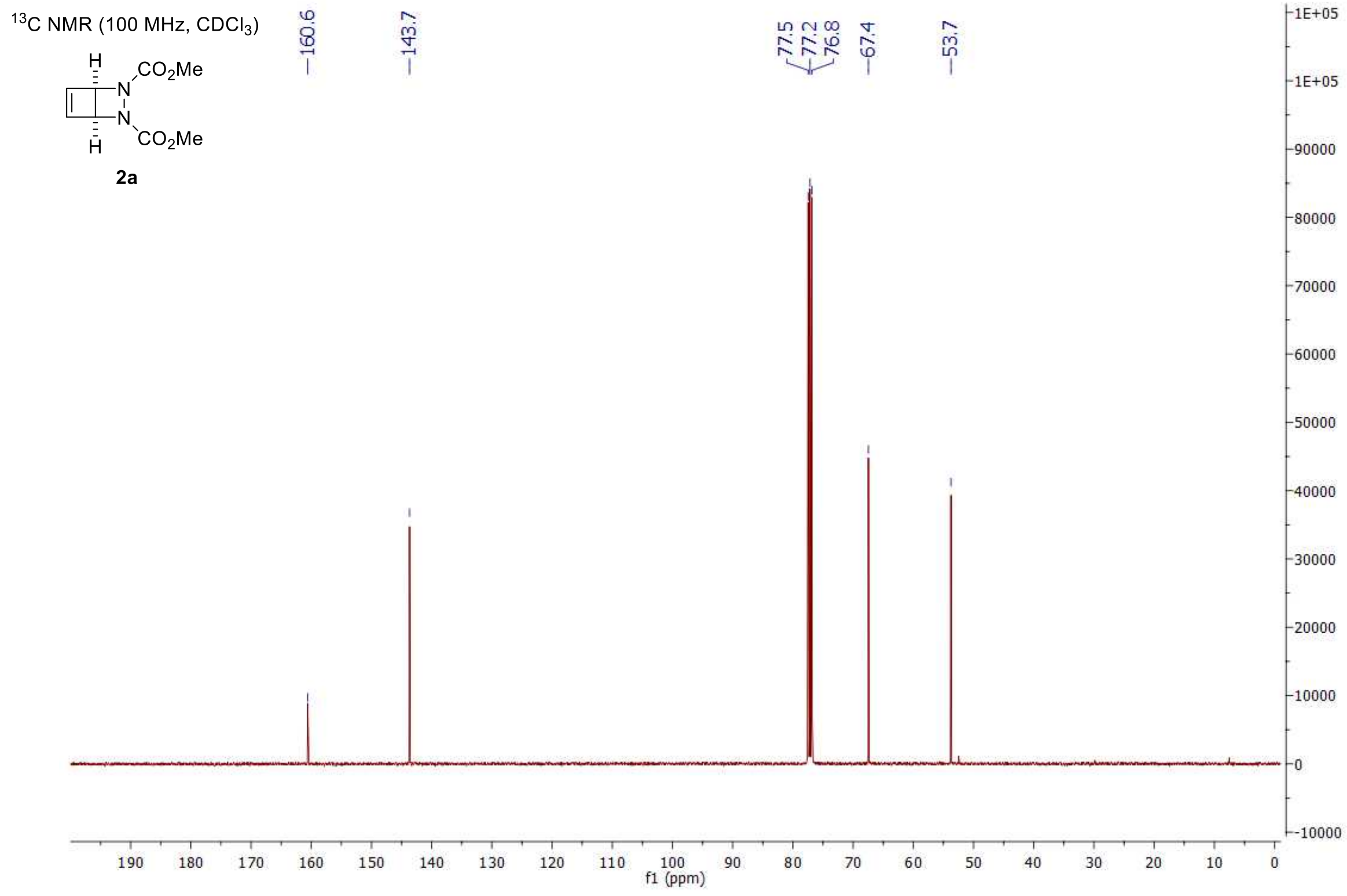




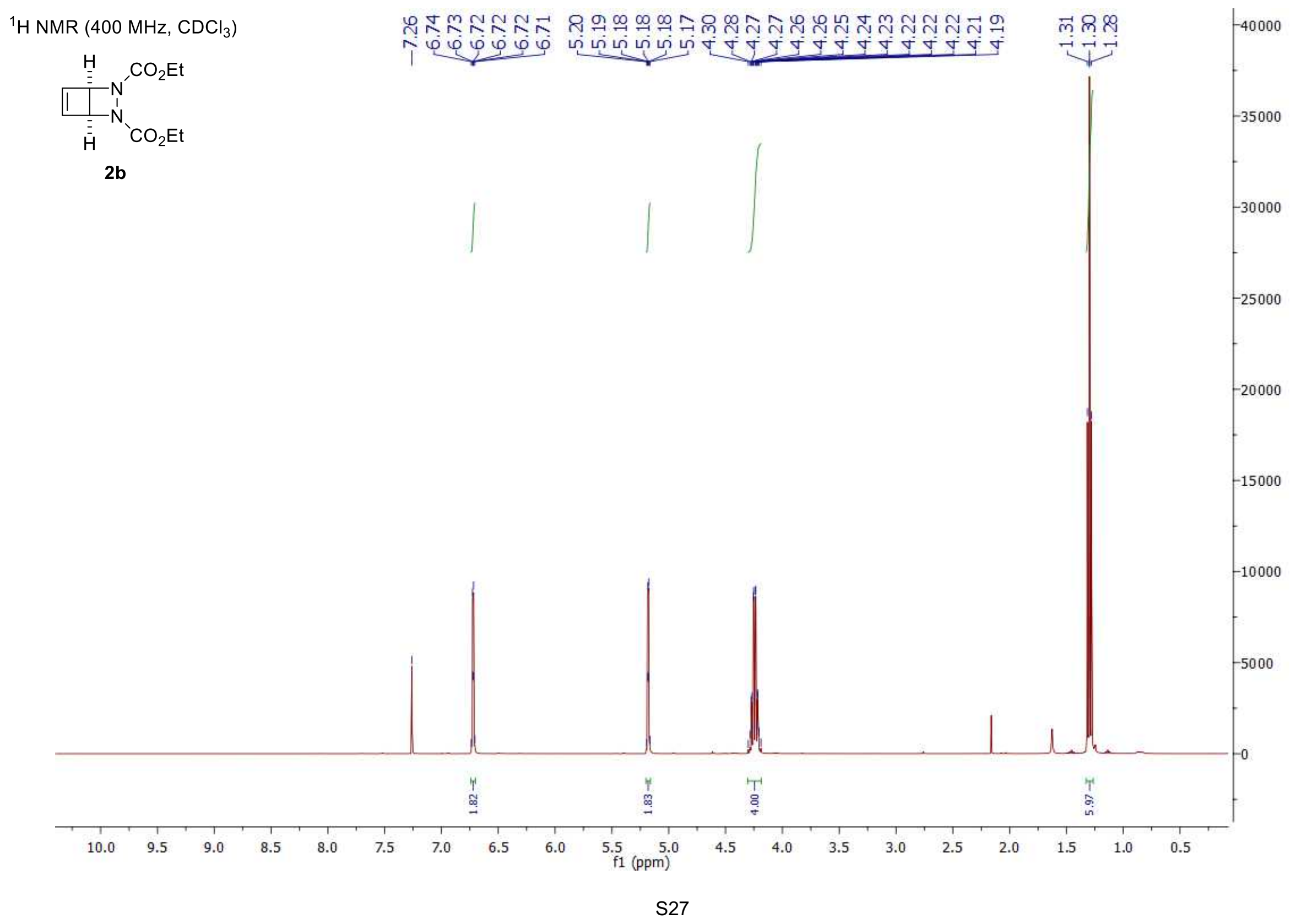




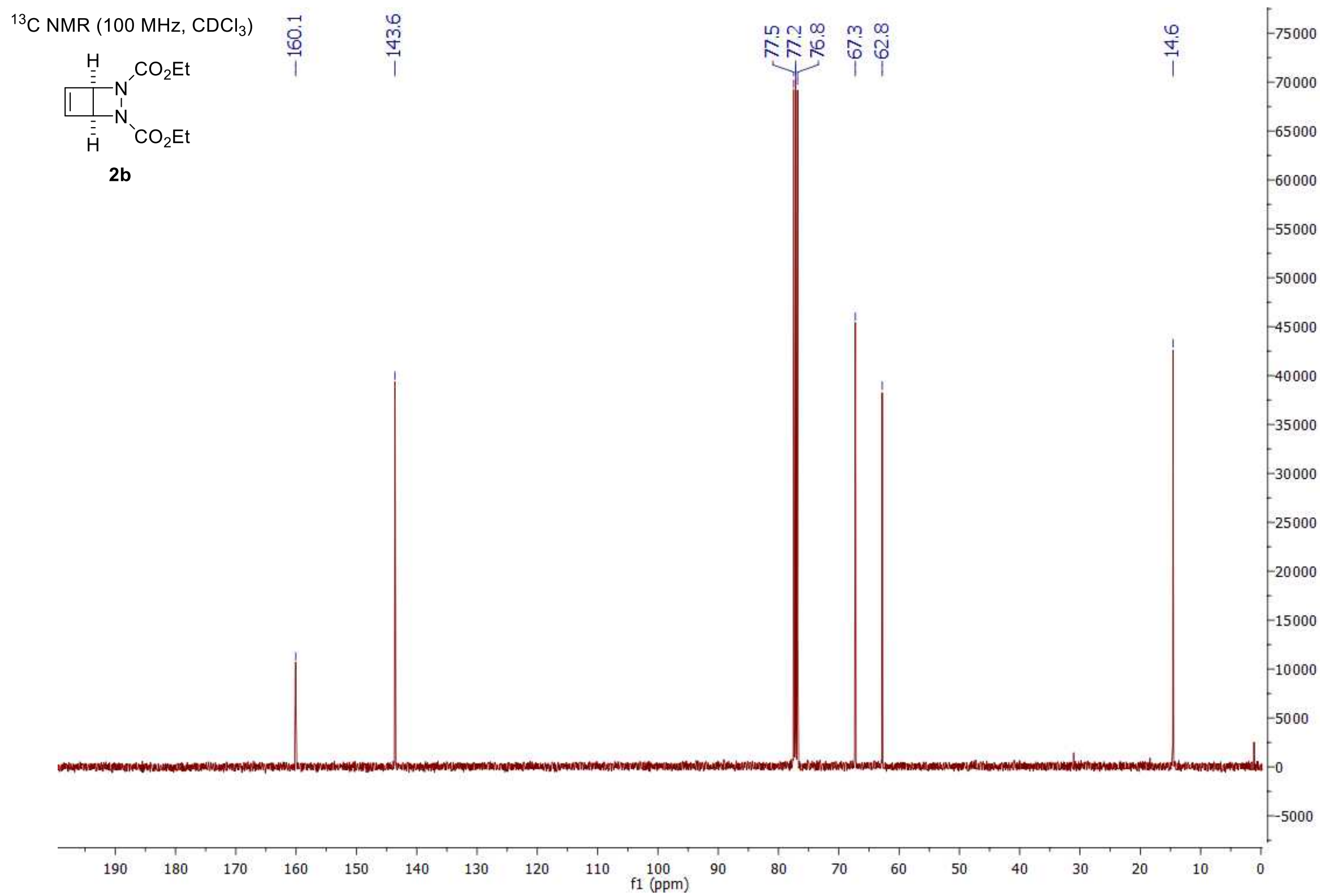




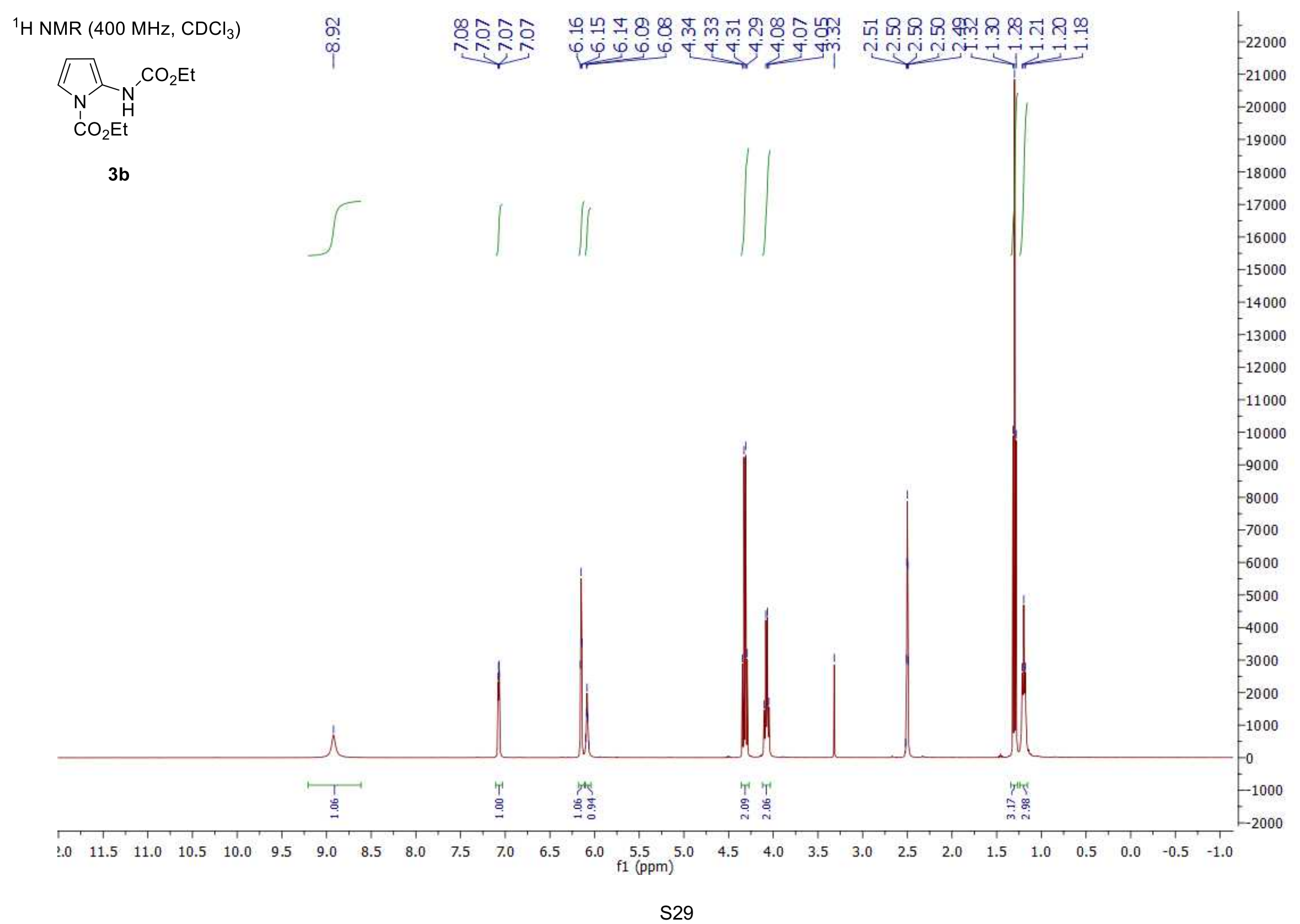




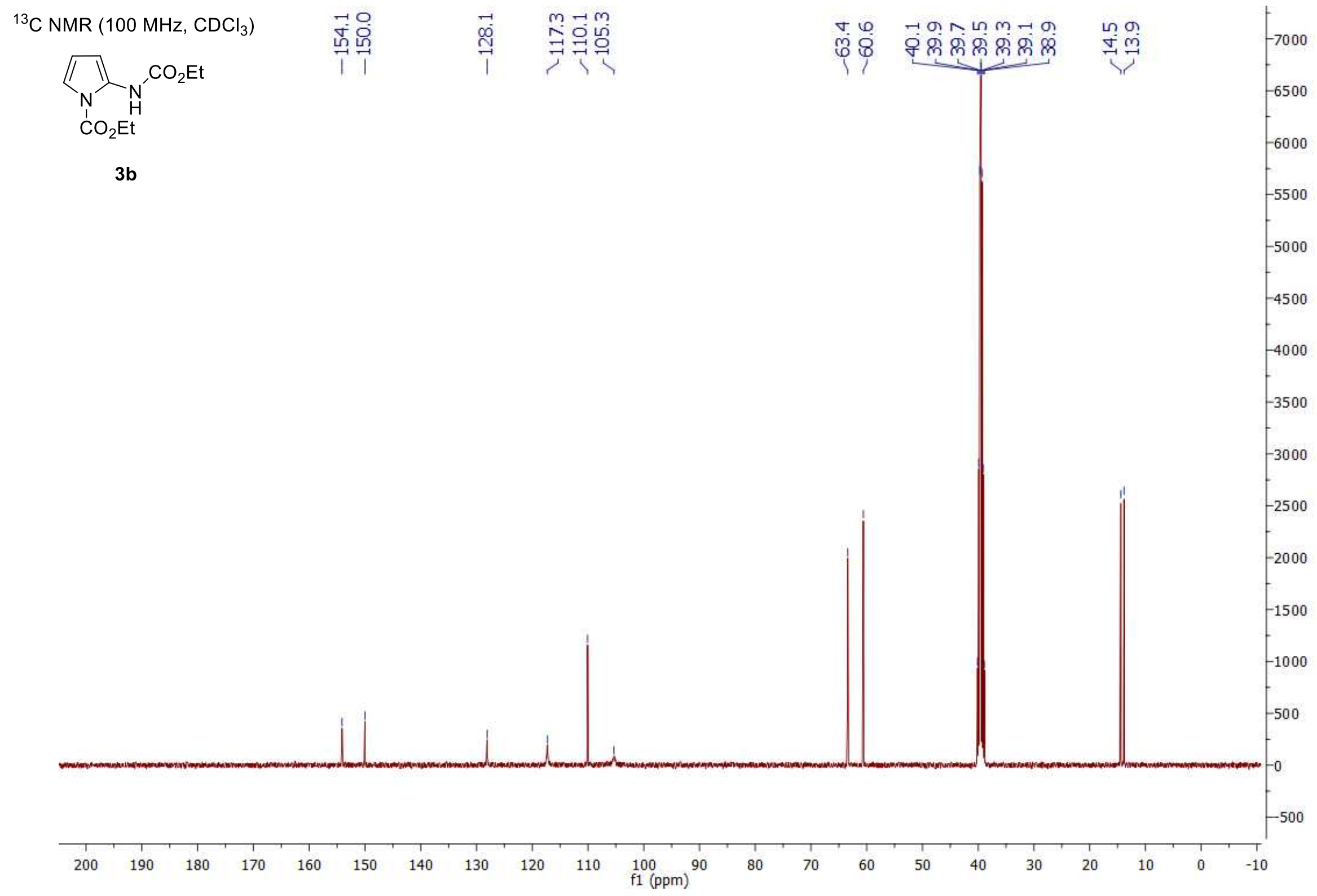




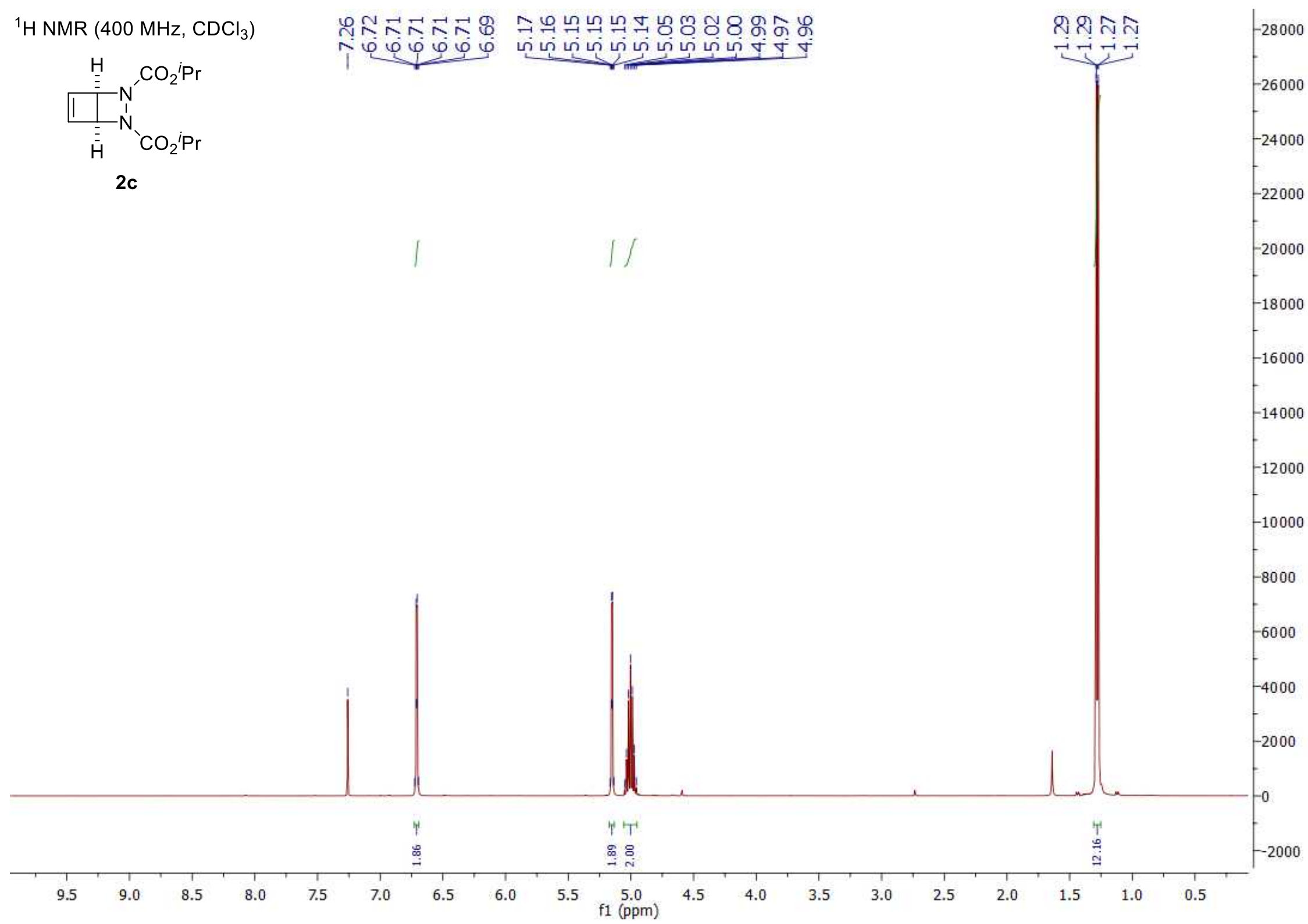




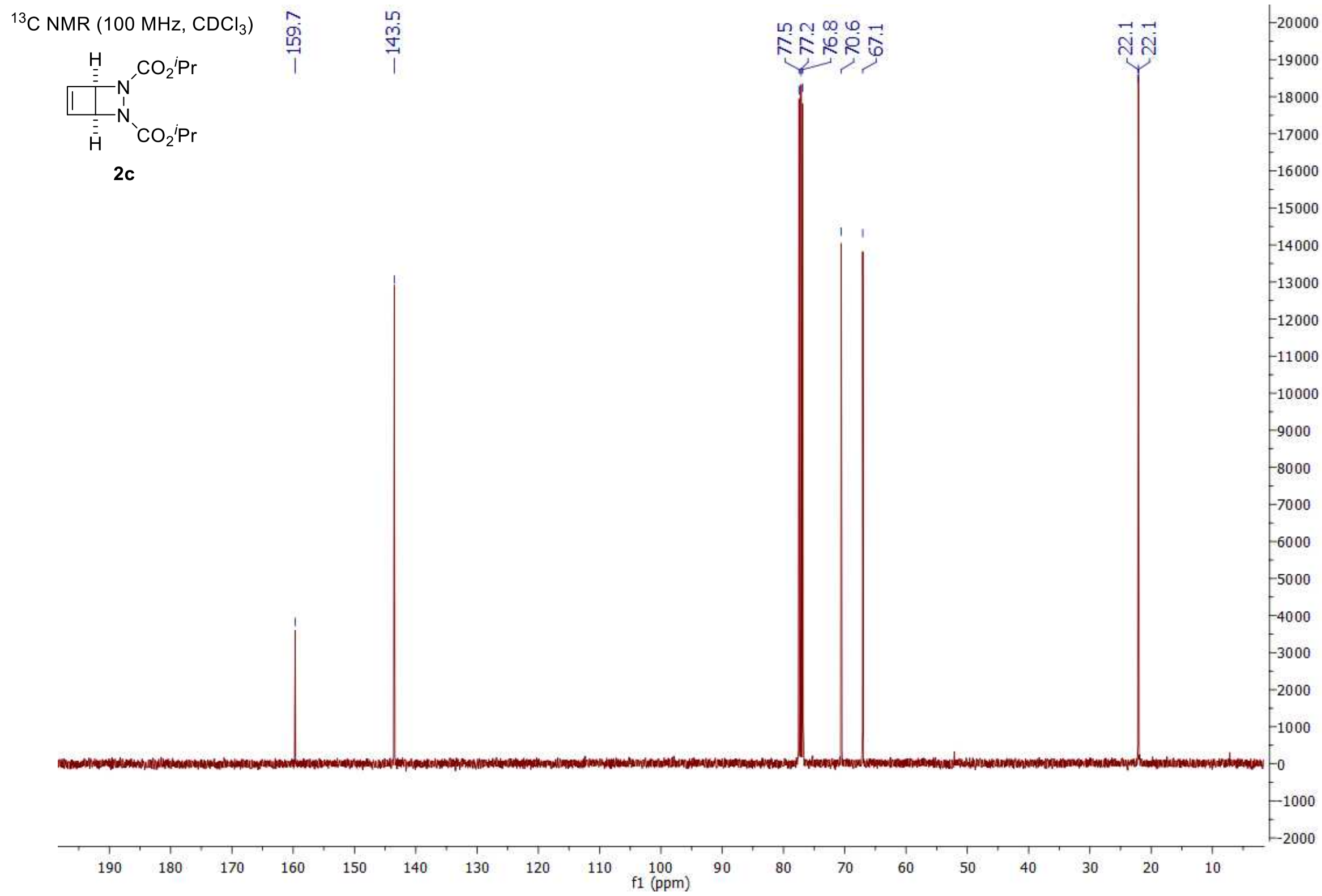




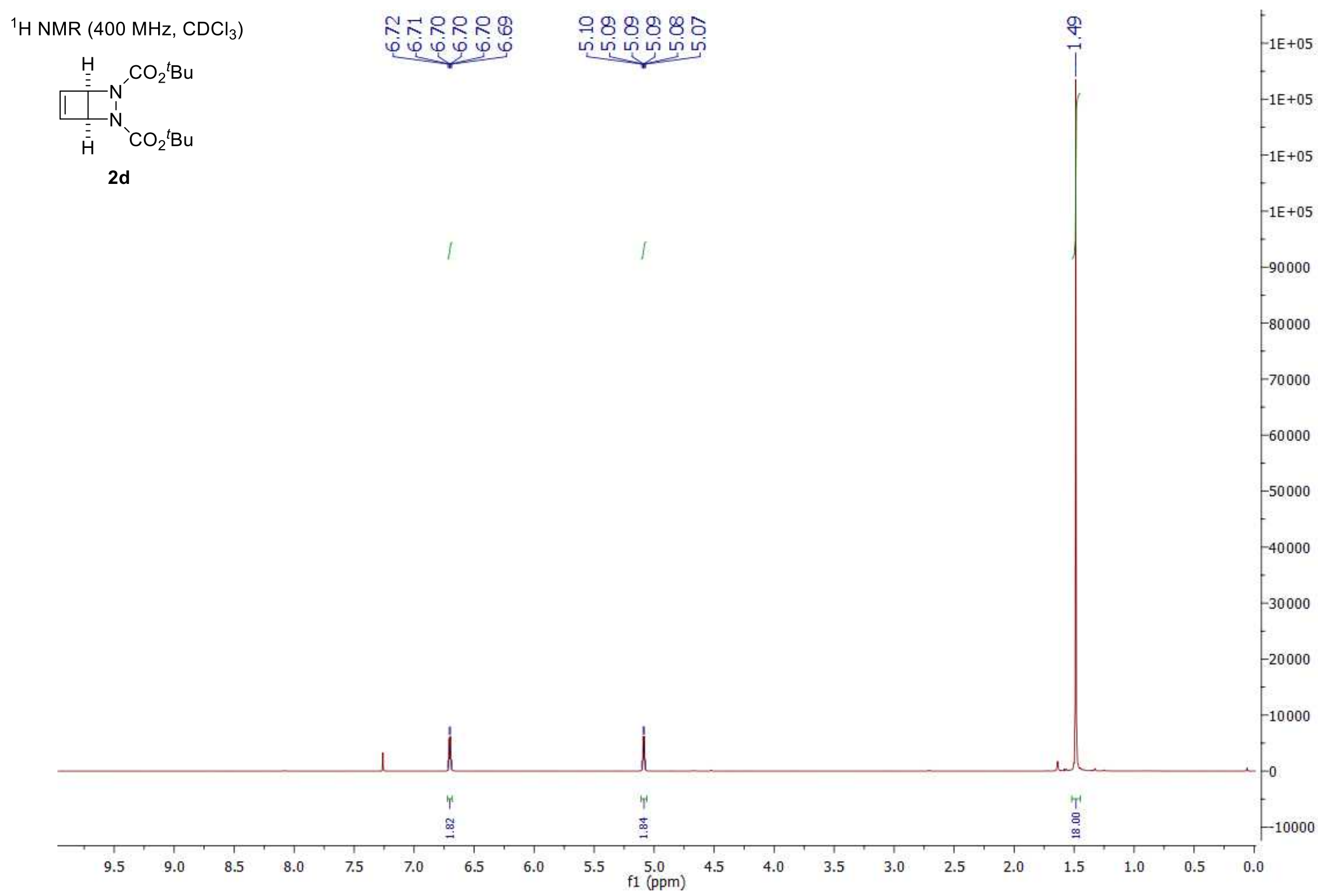




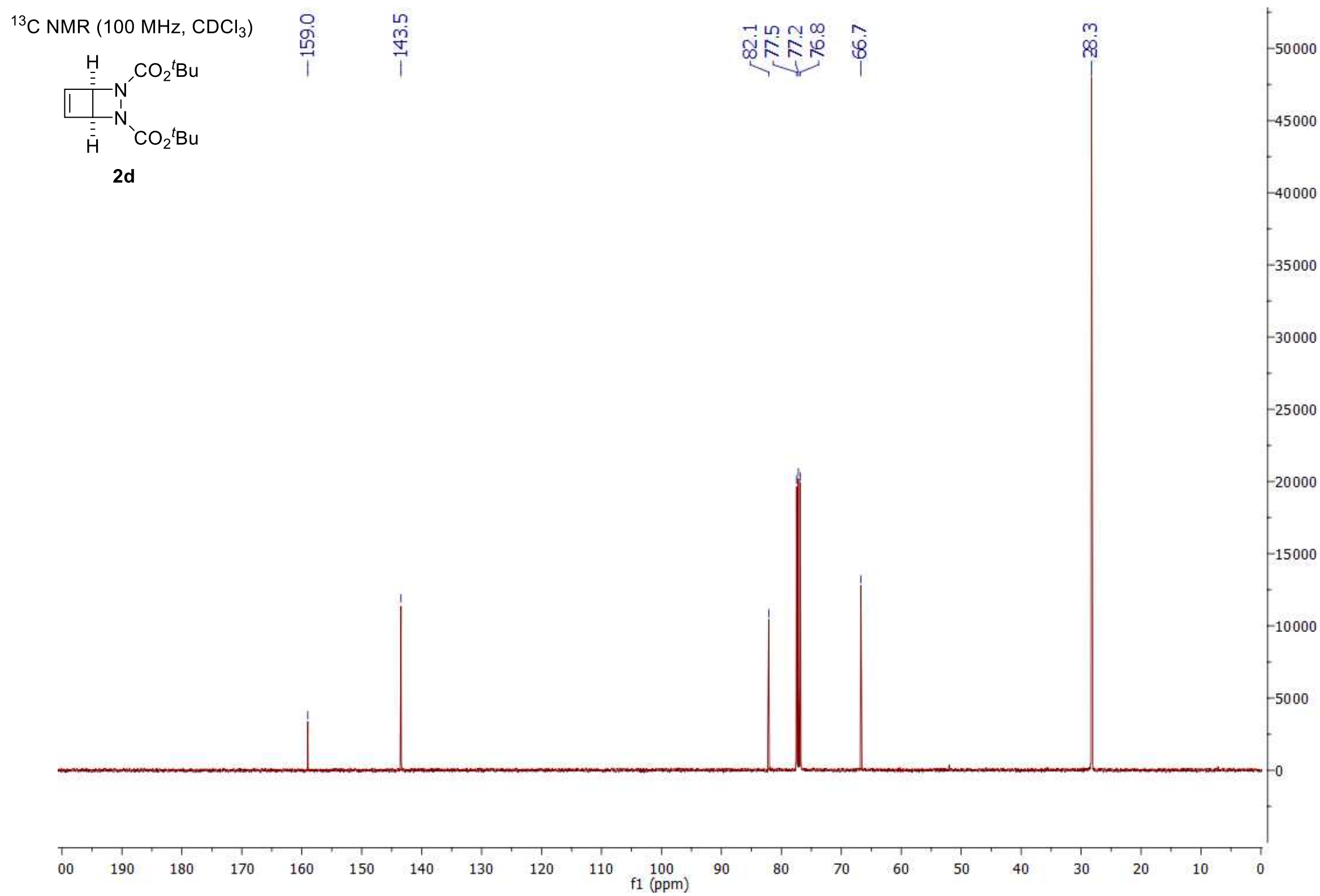




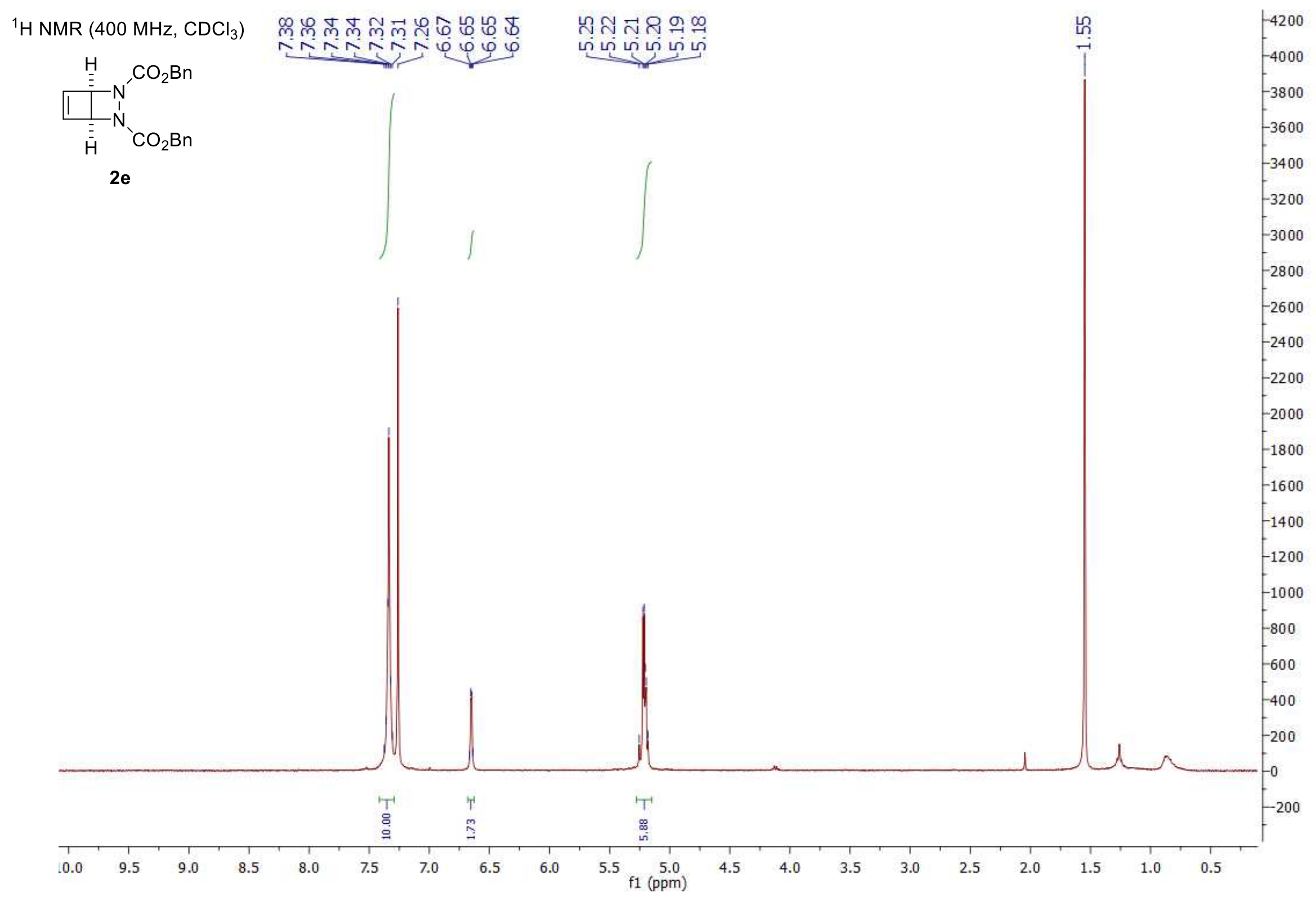




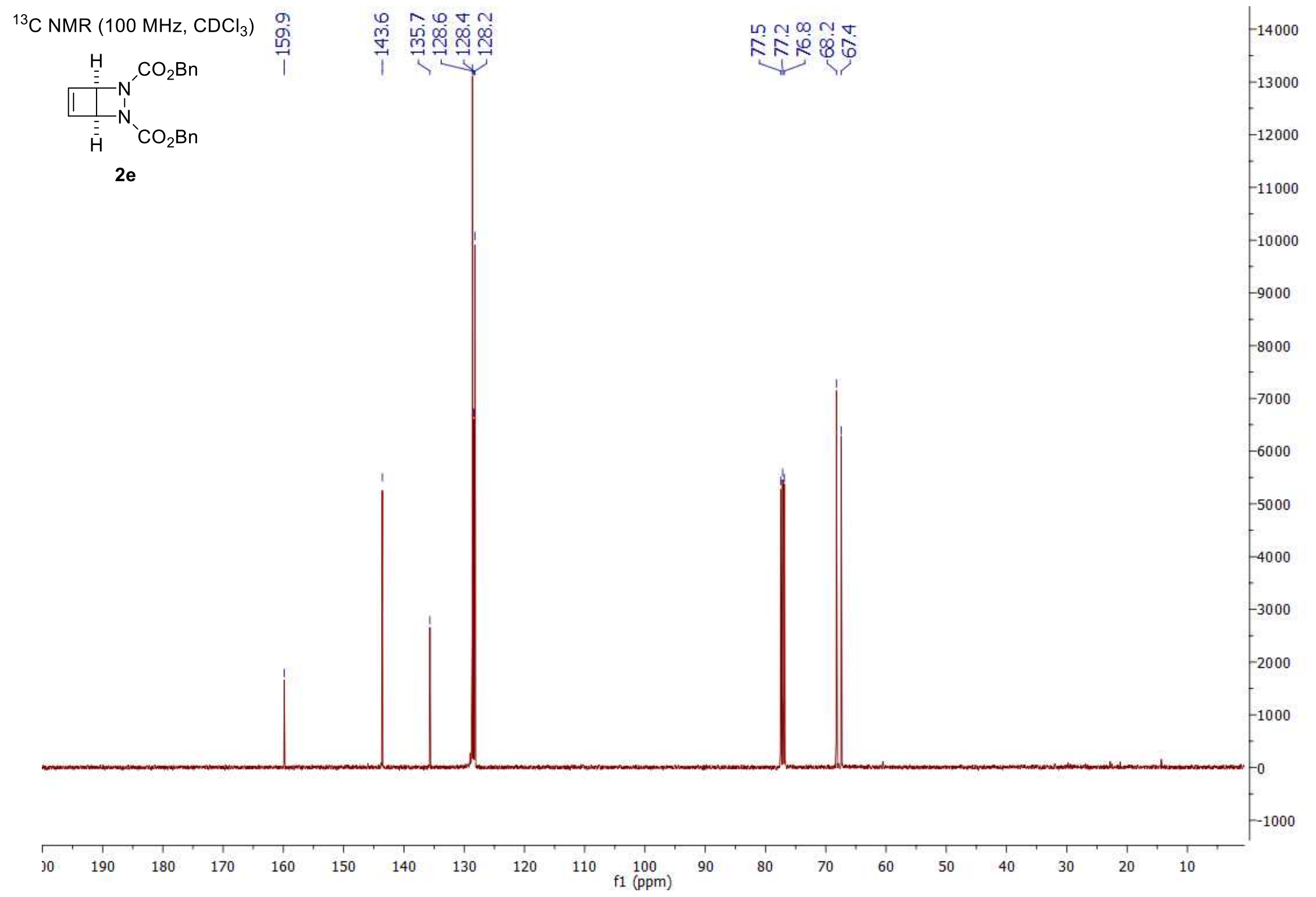




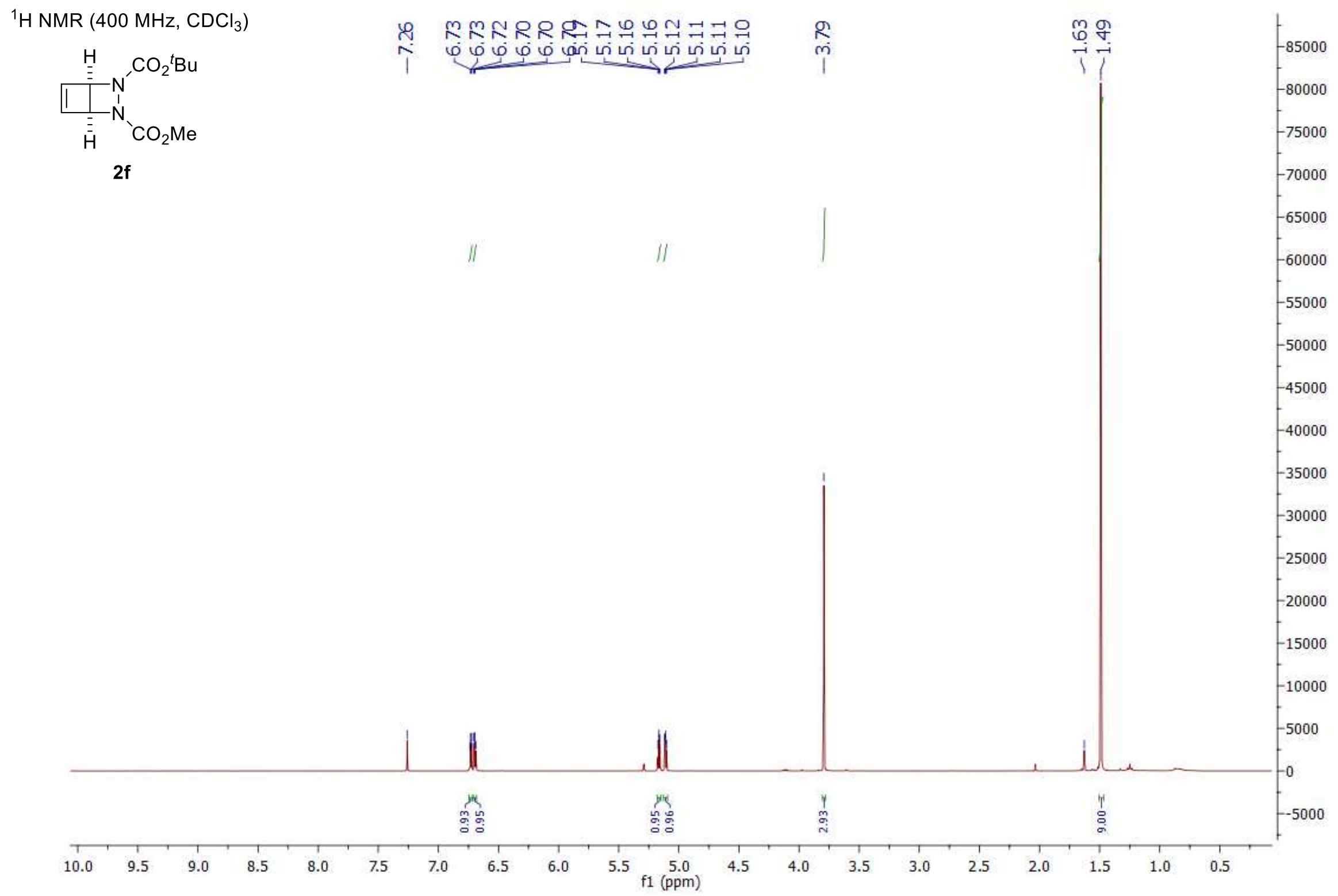




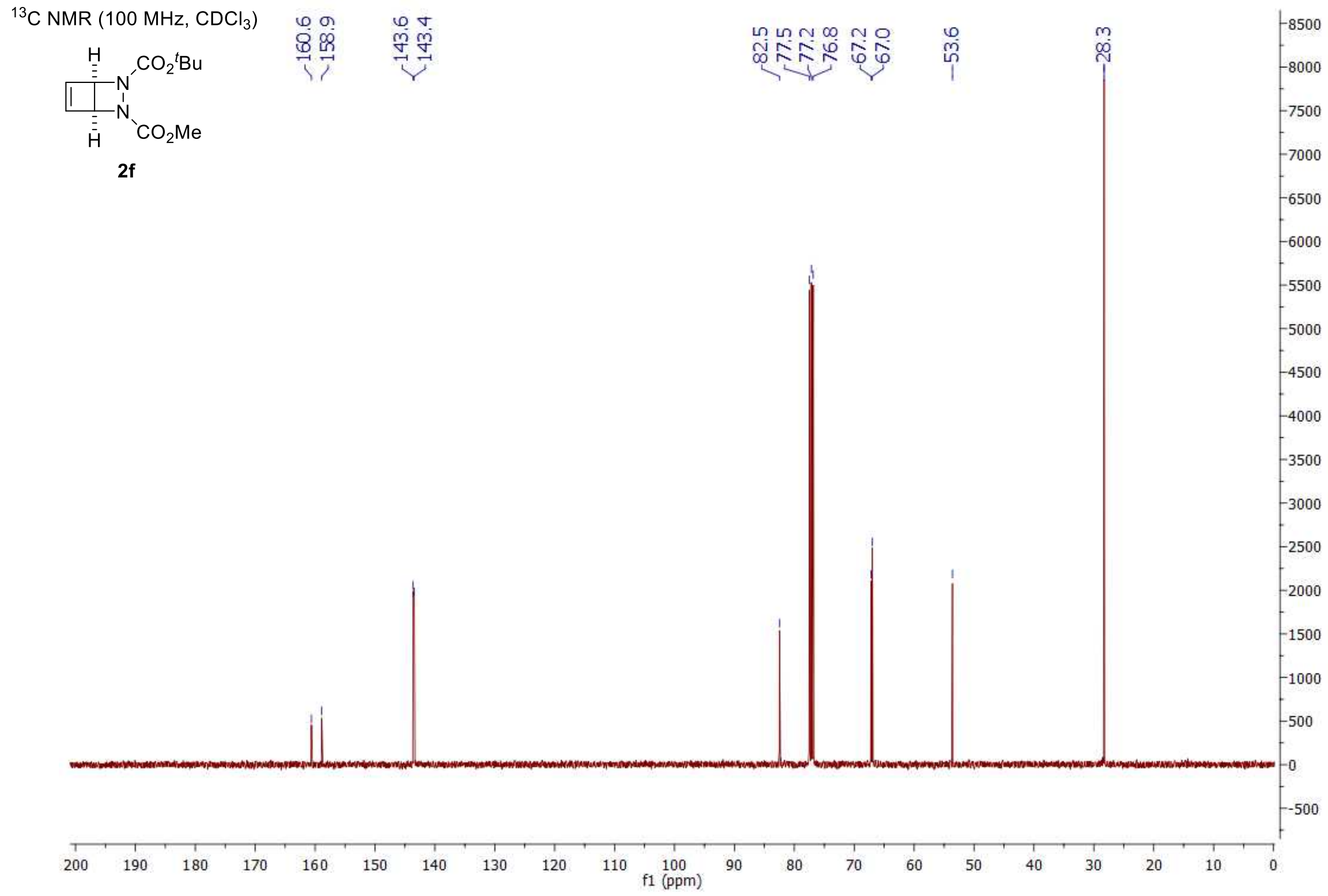




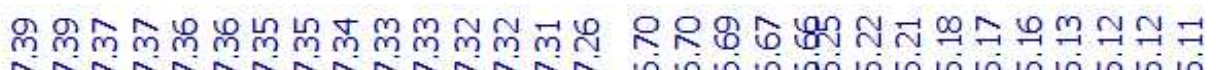

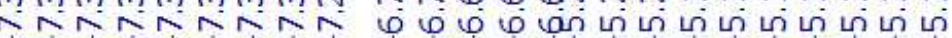

${ }^{1} \mathrm{H}$ NMR $\left(400 \mathrm{MHz}, \mathrm{CDCl}_{3}\right)$

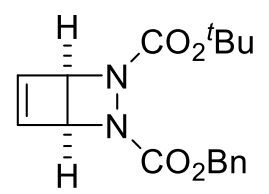

$2 g$
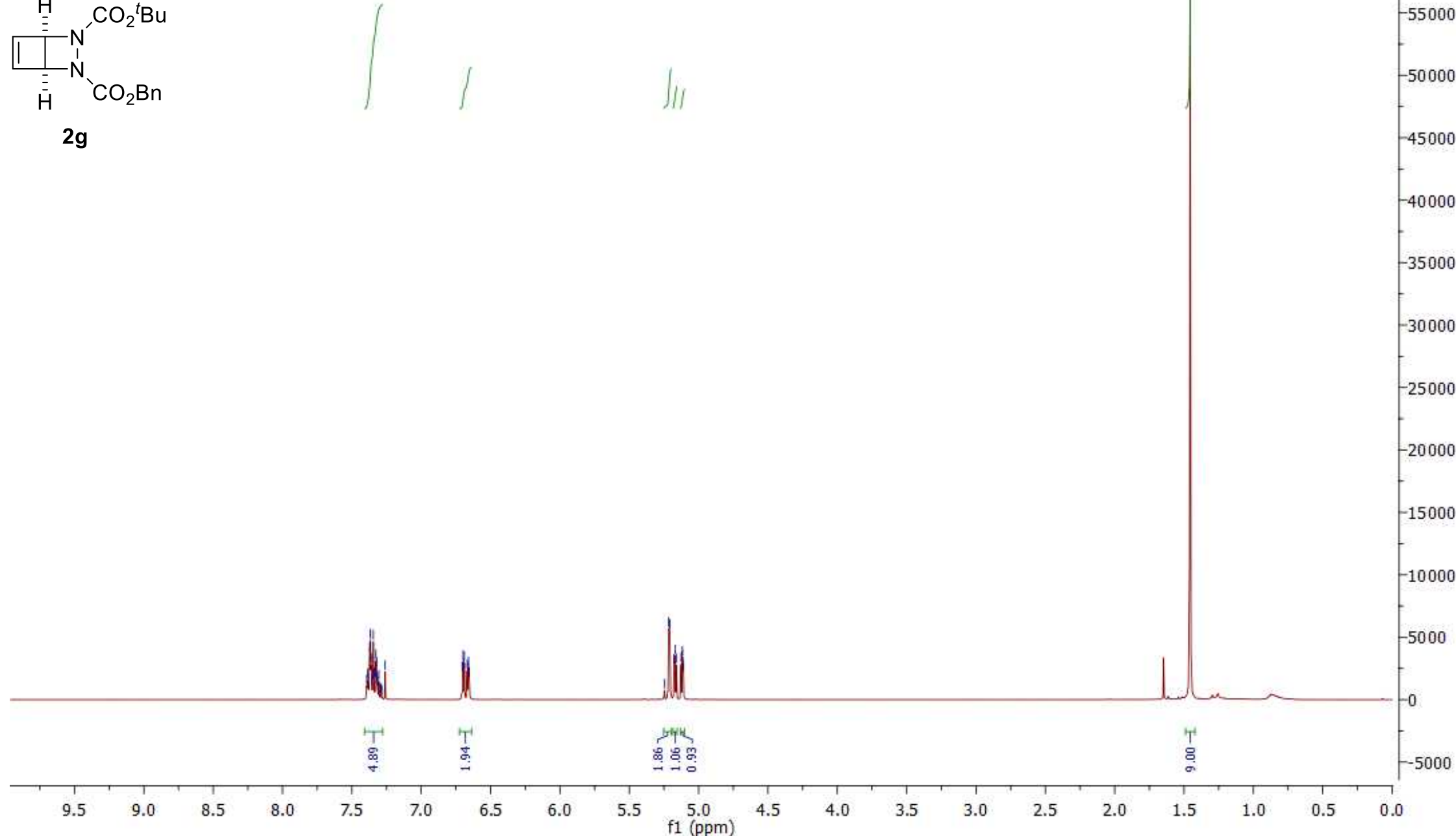


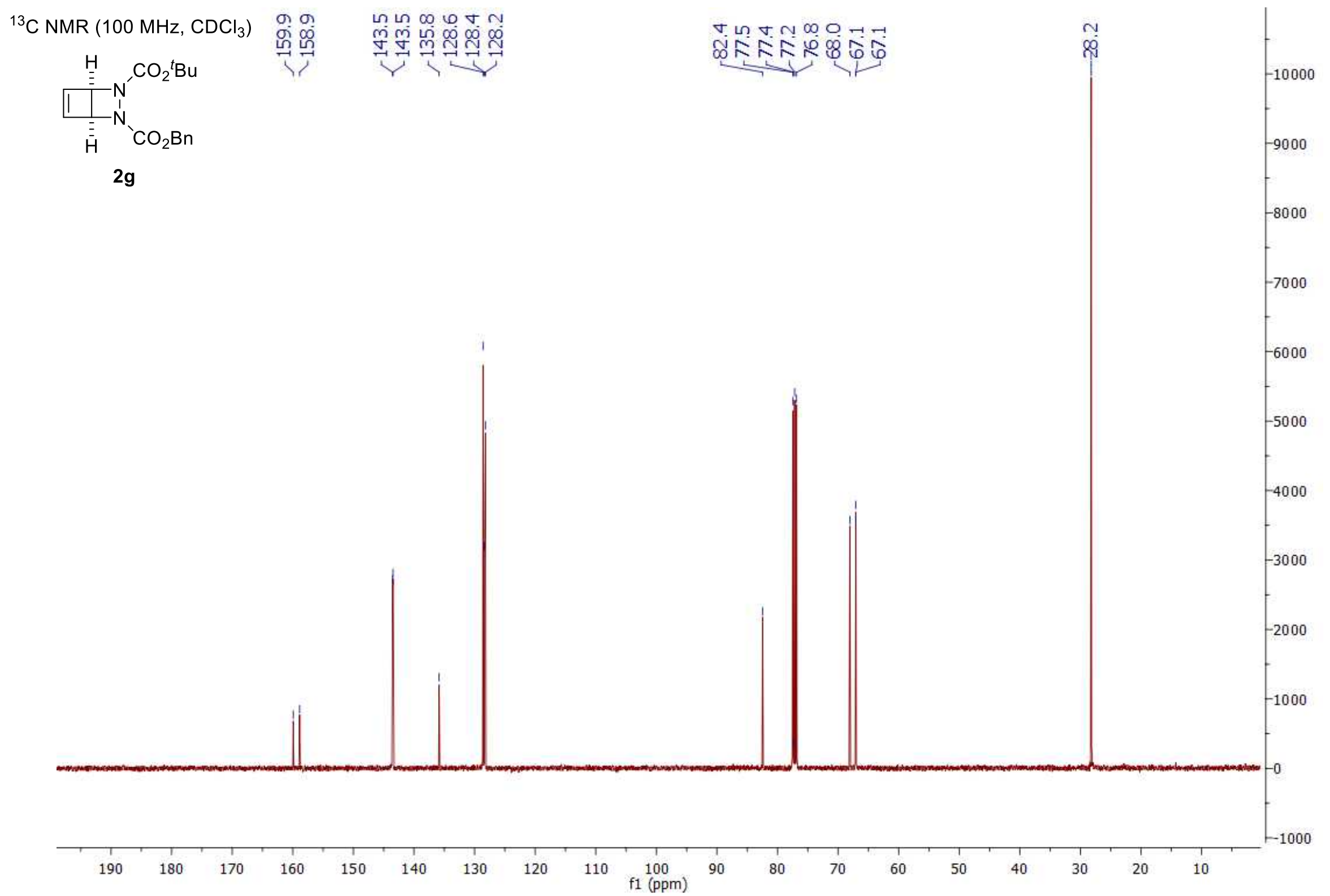




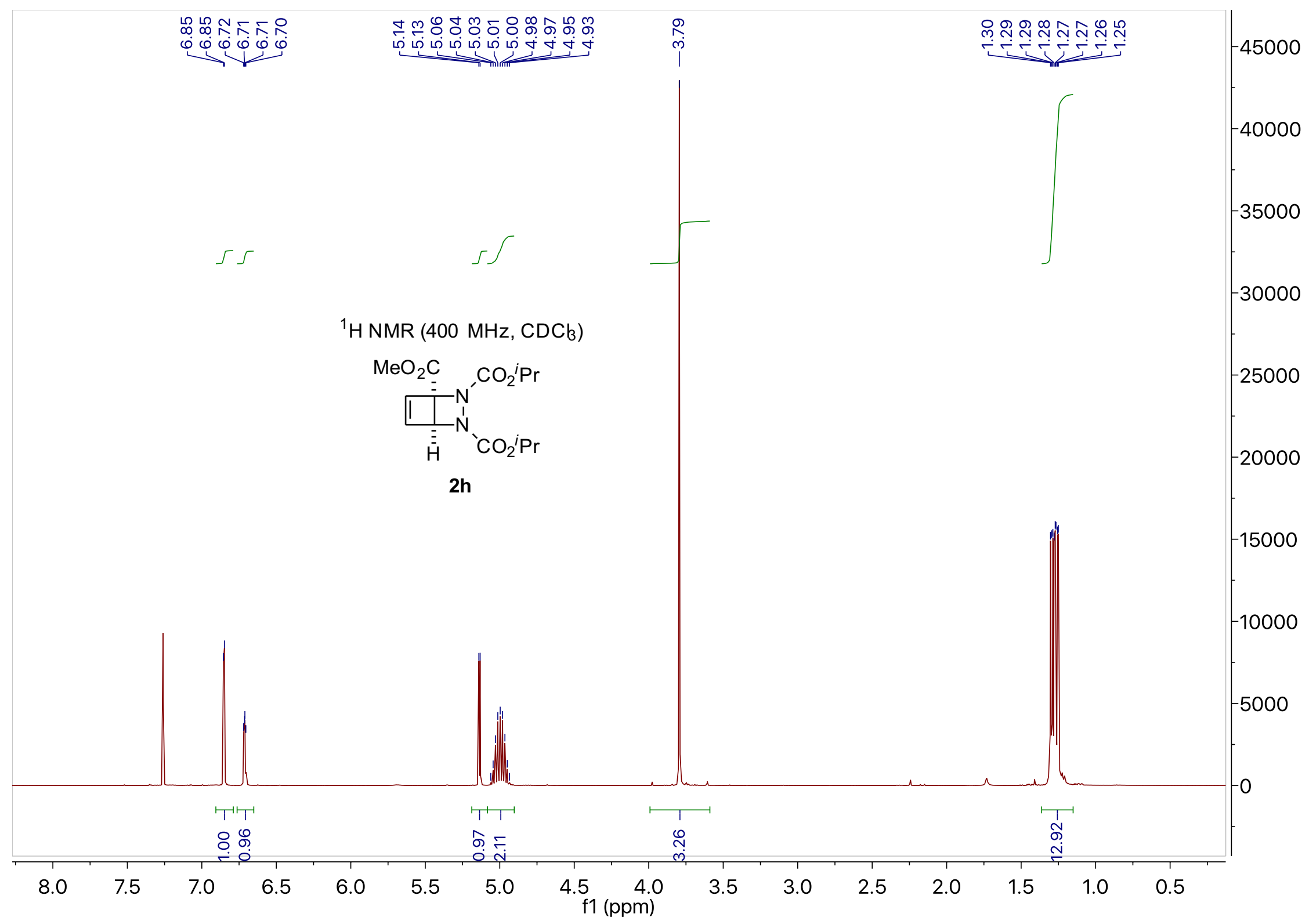




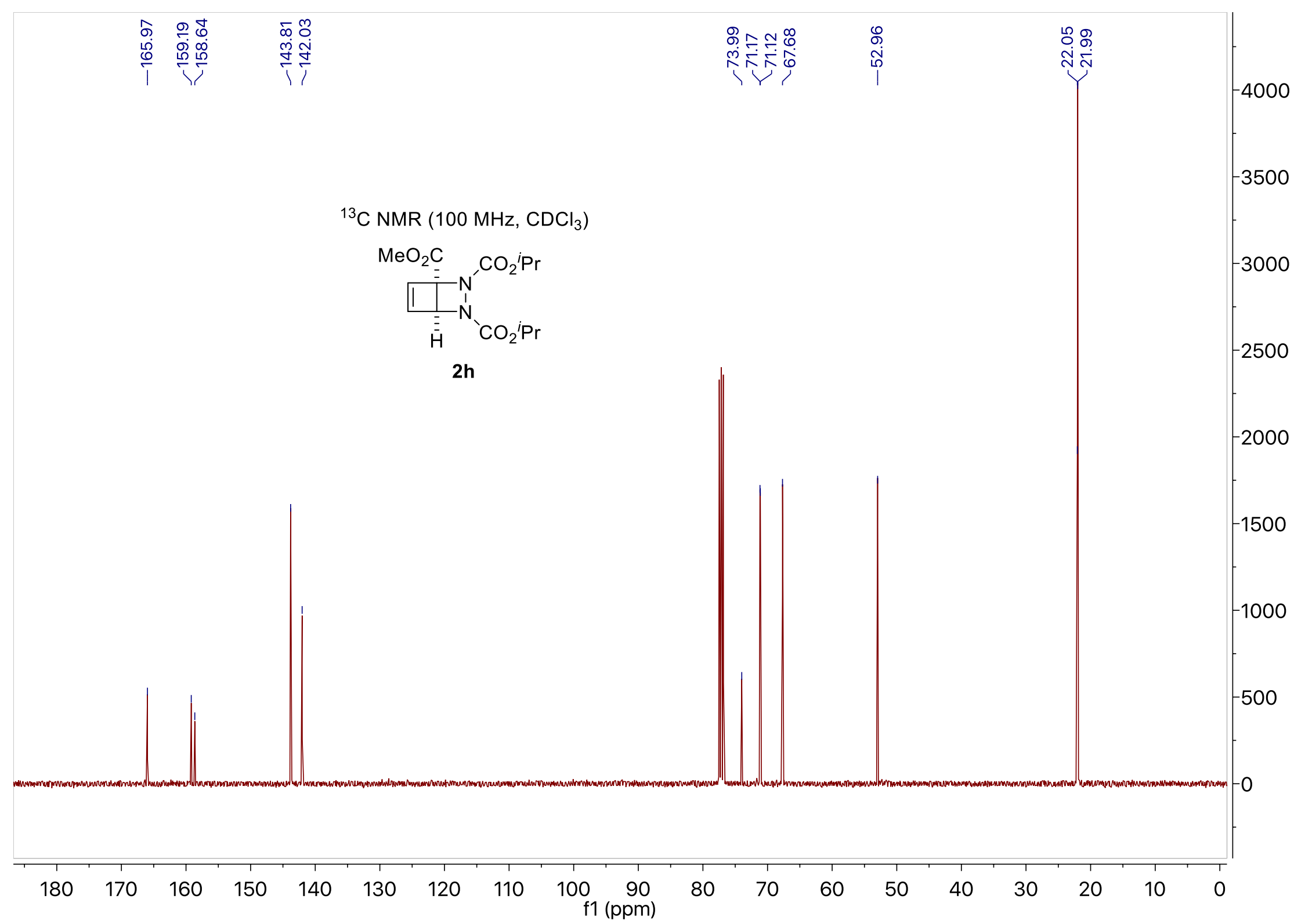




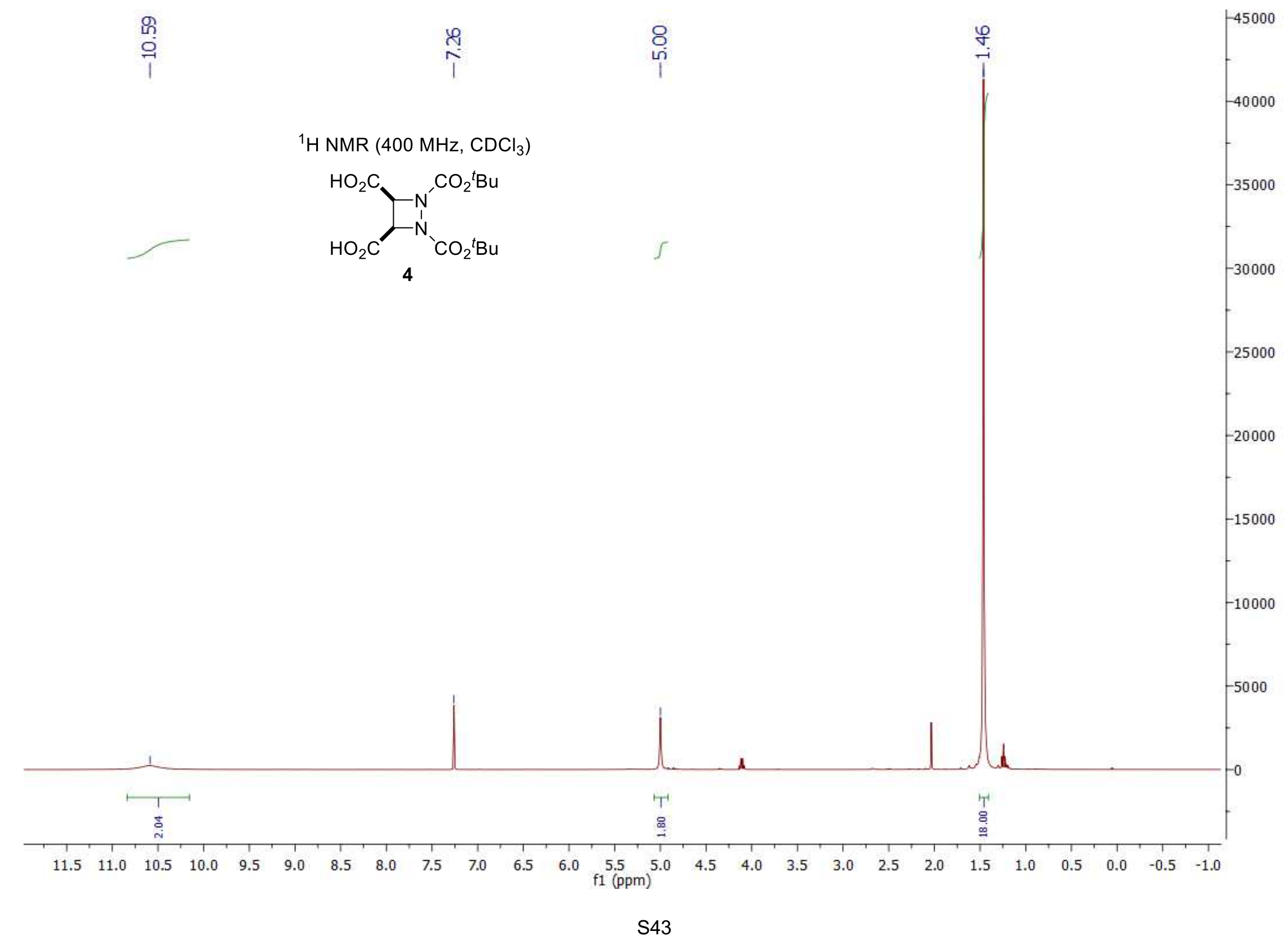


${ }^{13} \mathrm{C} \mathrm{NMR}\left(100 \mathrm{MHz}, \mathrm{CDCl}_{3}\right)$
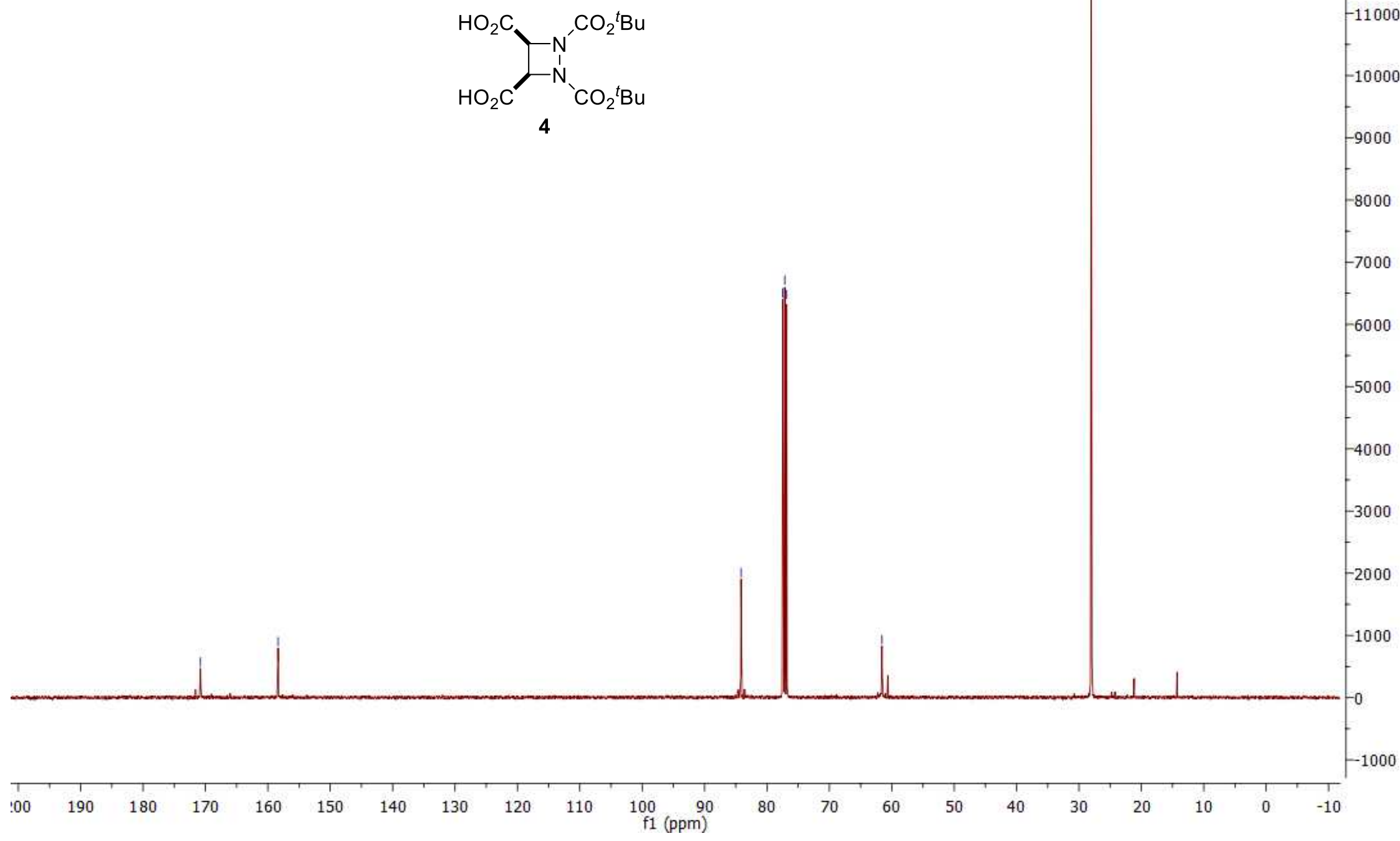

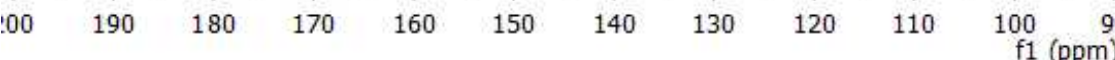




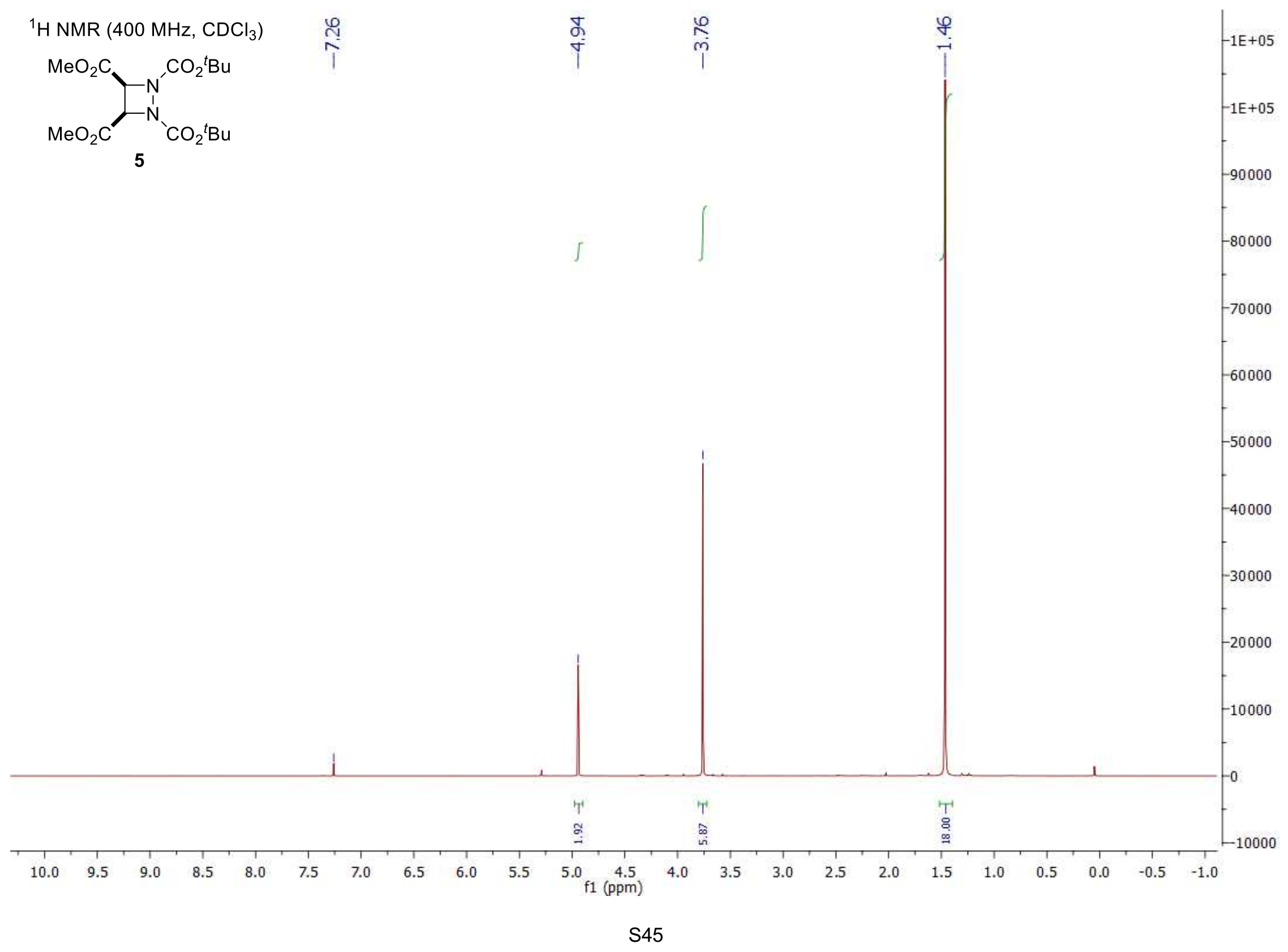




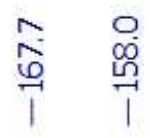

mNํํํำ

${ }^{13} \mathrm{C}$ NMR (100 MHz, $\mathrm{CDCl}_{3}$ )

$\mathrm{MeO}_{2} \mathrm{C}$

$\mathrm{MeO}_{2} \mathrm{C}^{1}{ }^{\mathrm{N}} \mathrm{CO}_{2}{ }^{t} \mathrm{Bu}$

5

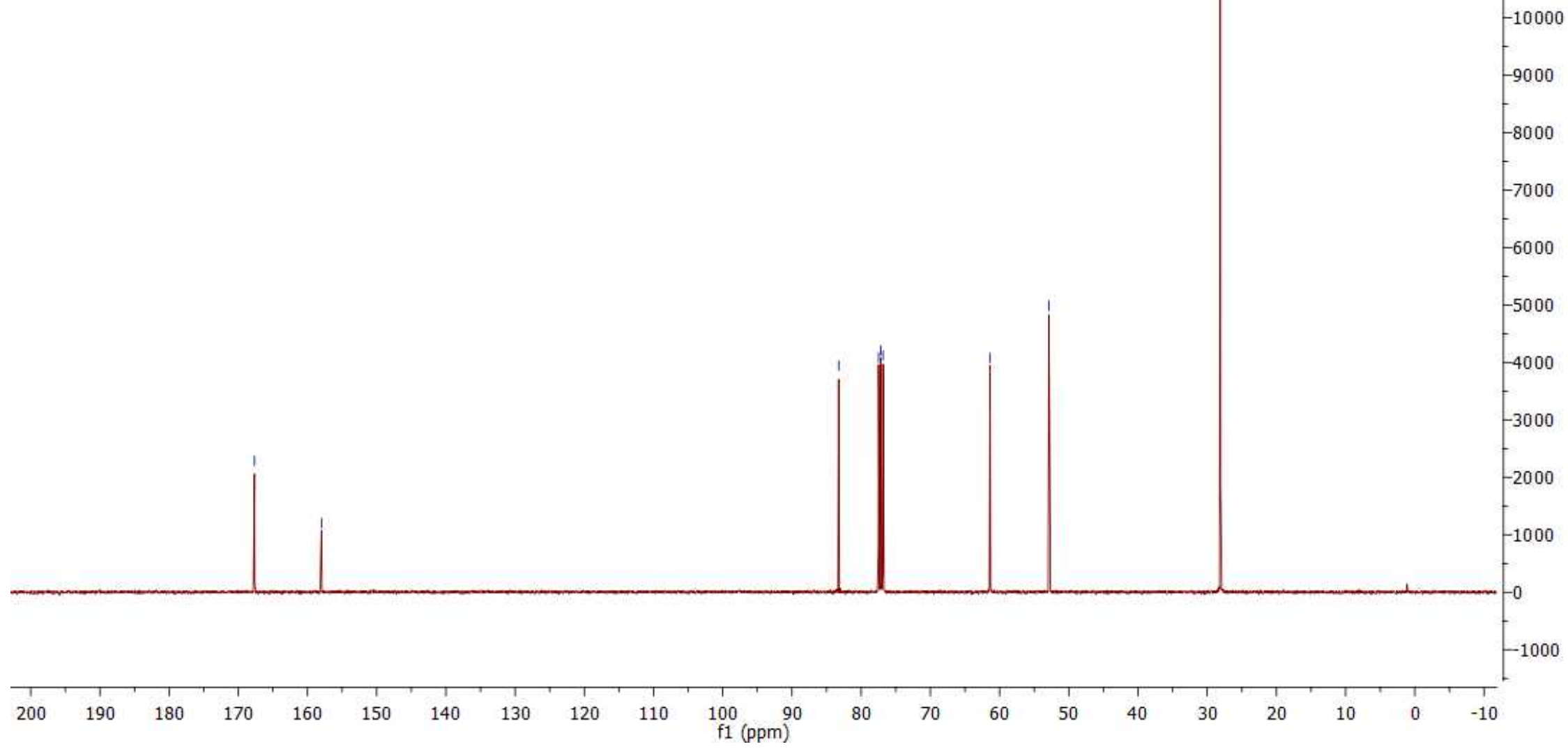




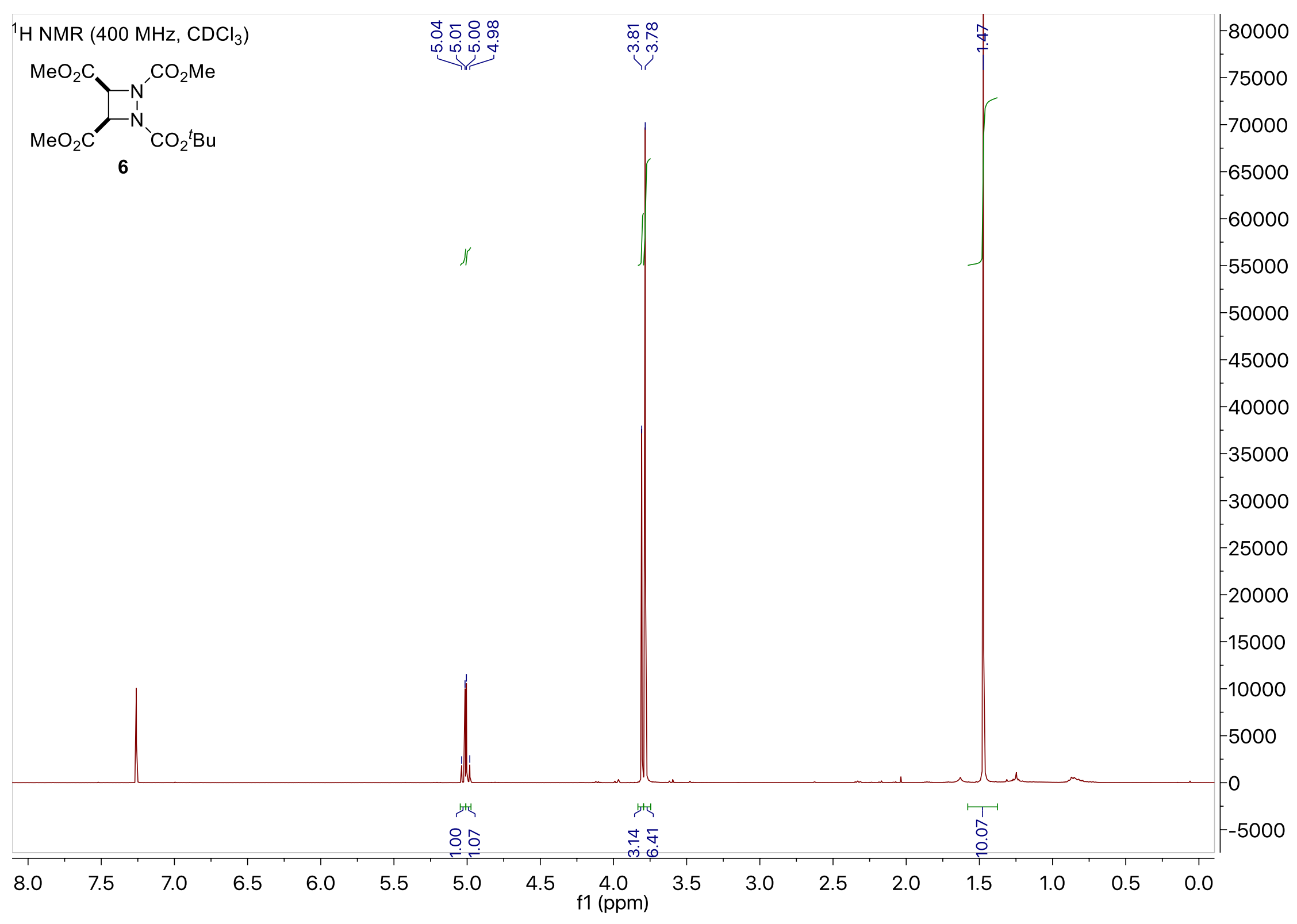




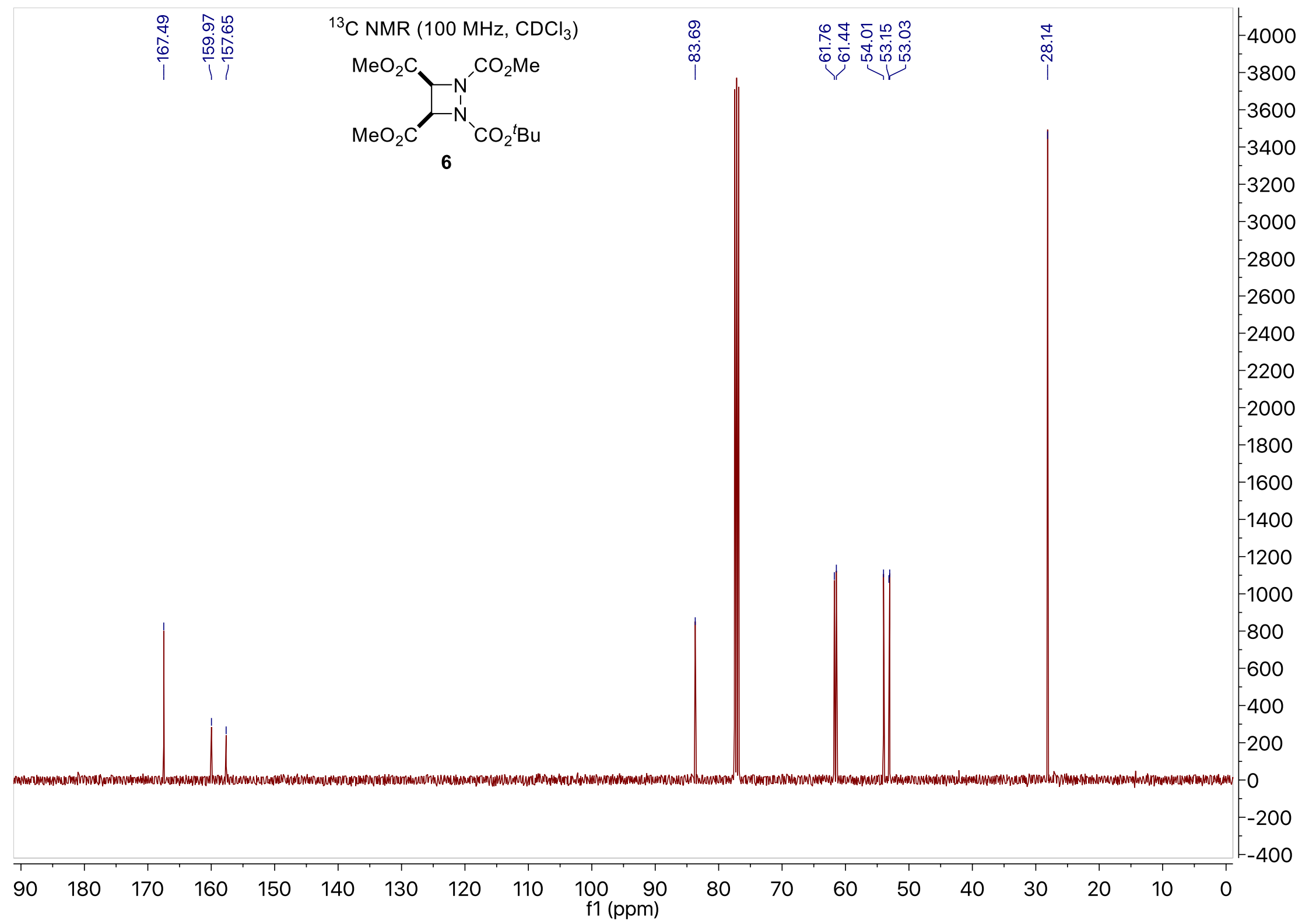




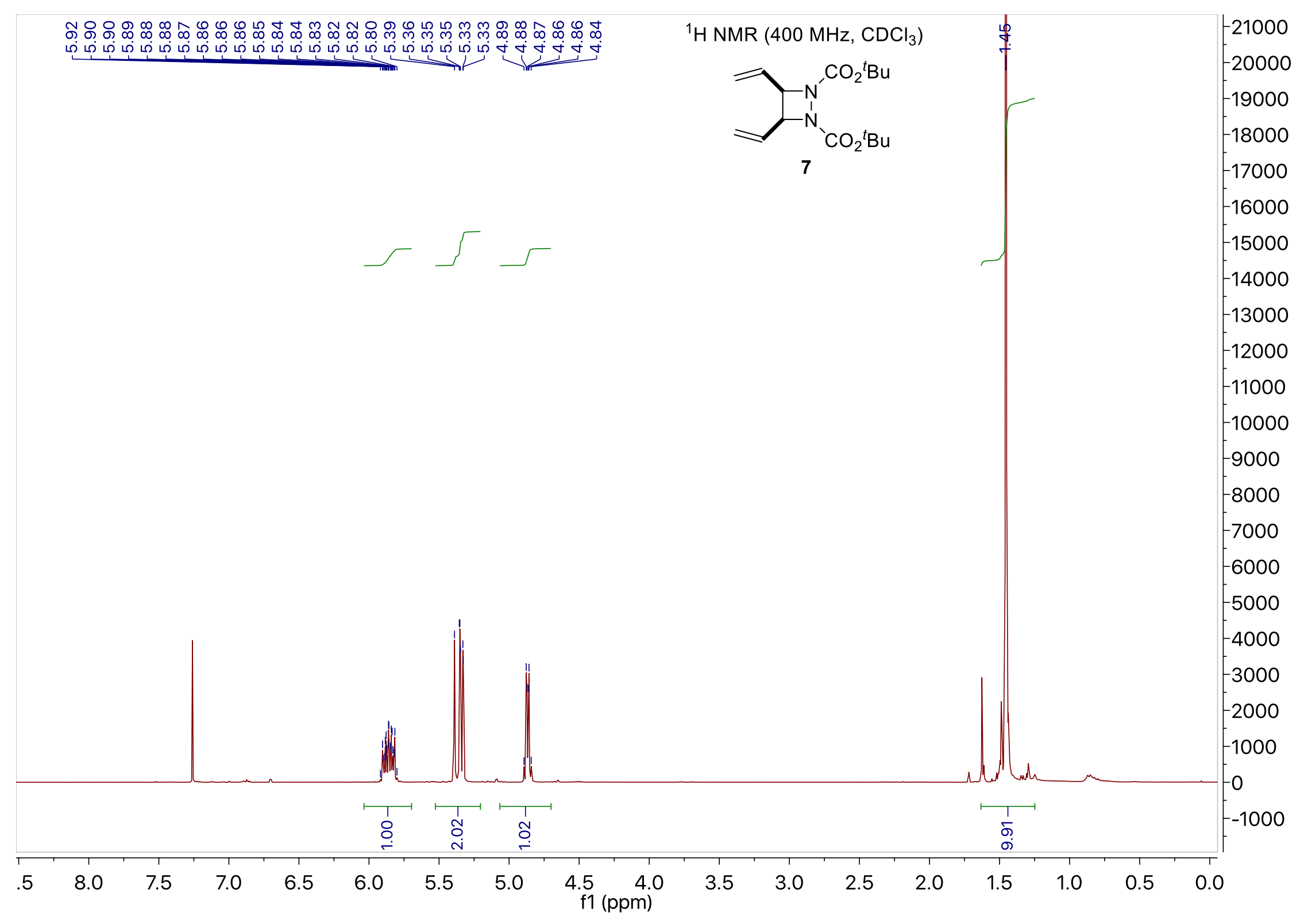




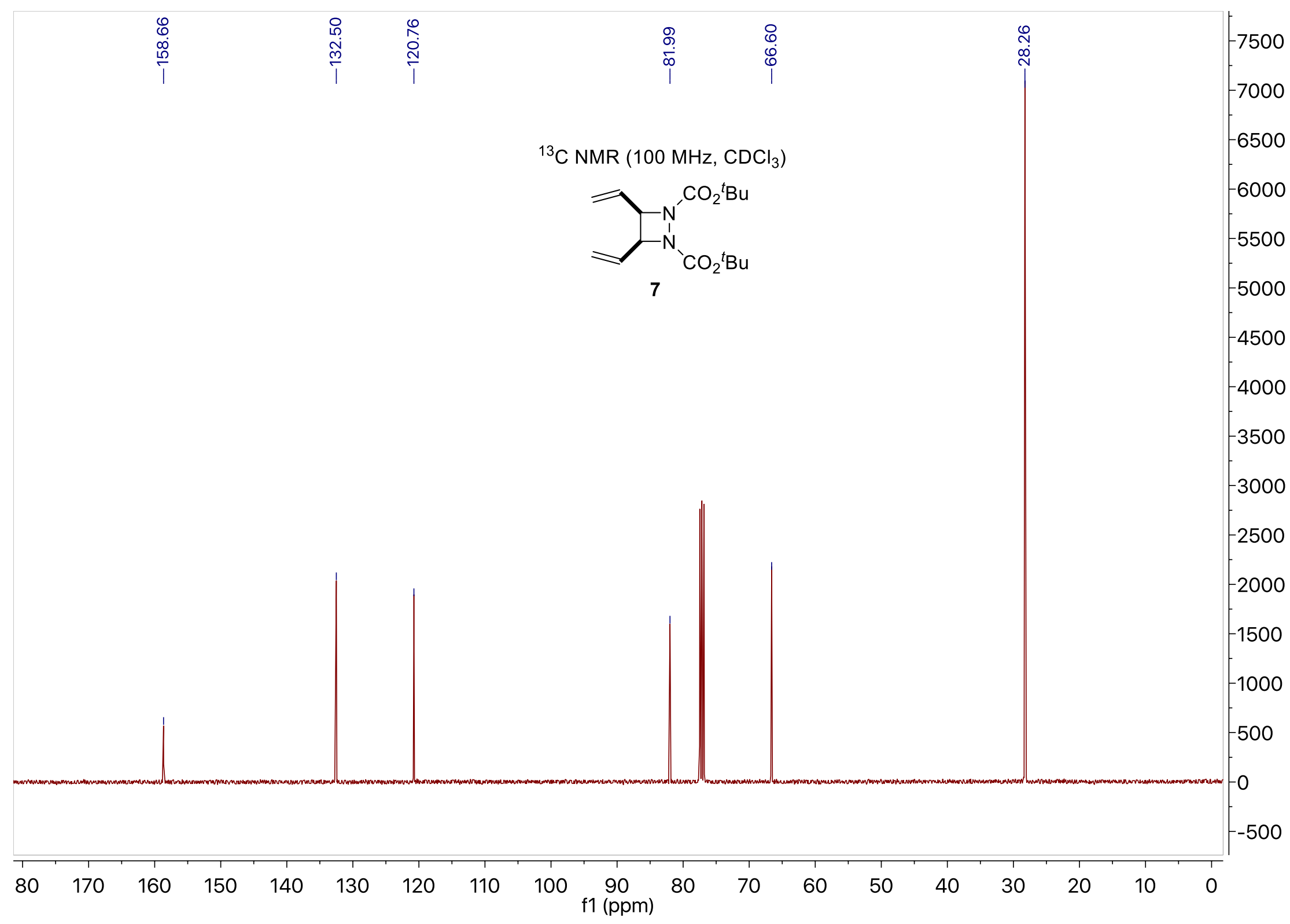




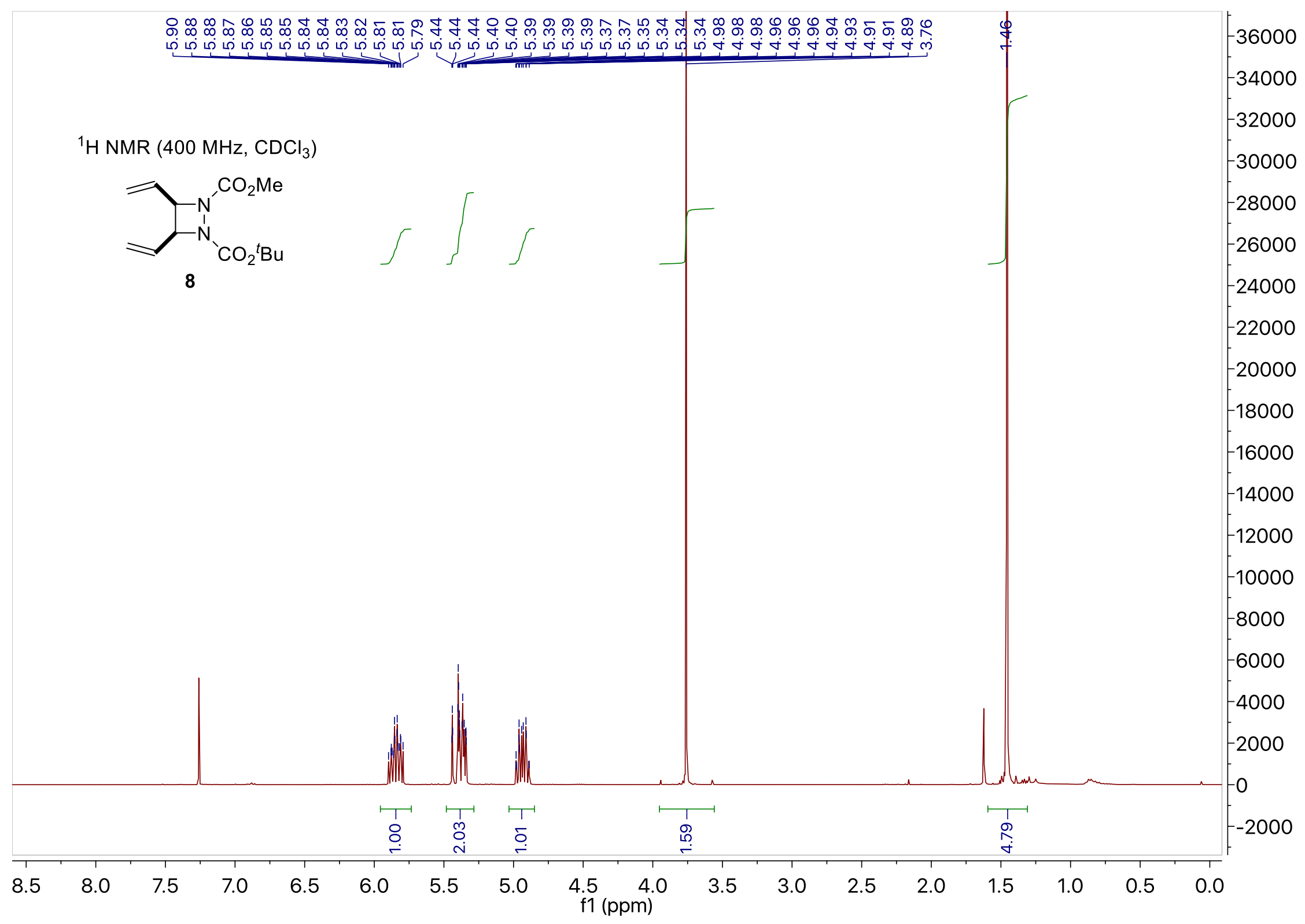




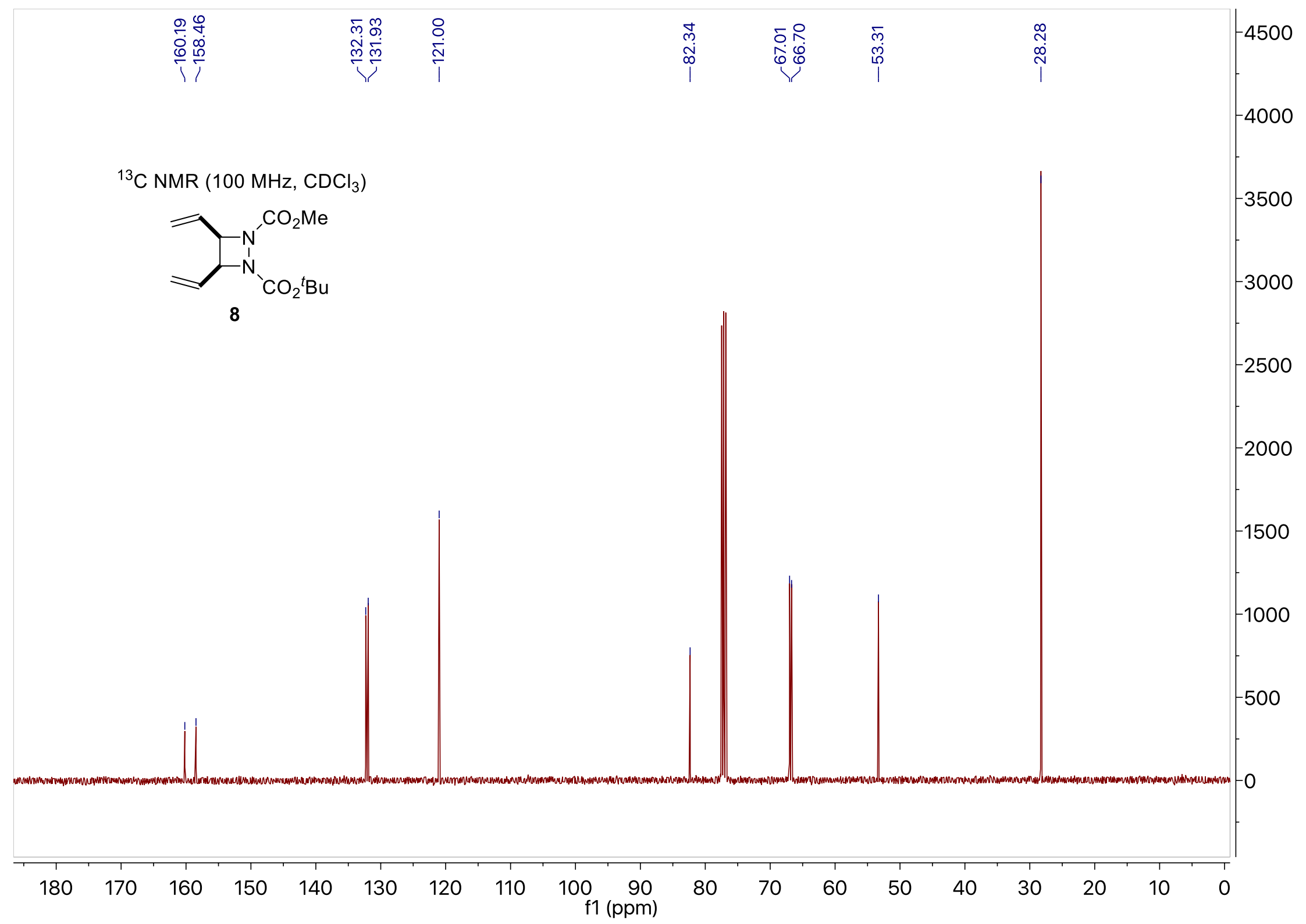


${ }^{1} \mathrm{H} \mathrm{NMR}\left(400 \mathrm{MHz}, \mathrm{CDCl}_{3}\right)$

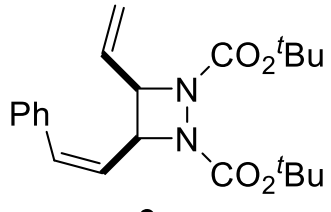

9a
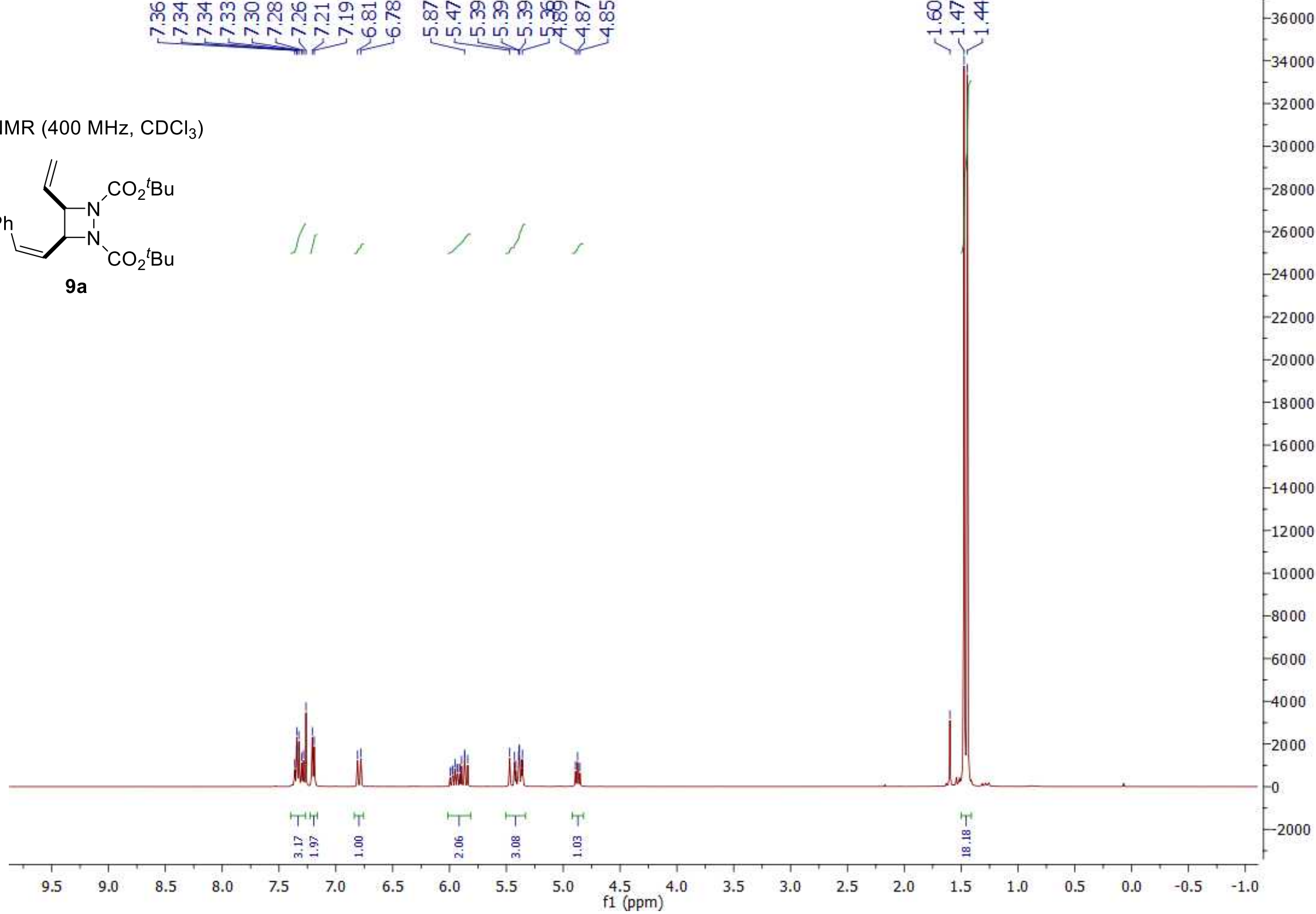


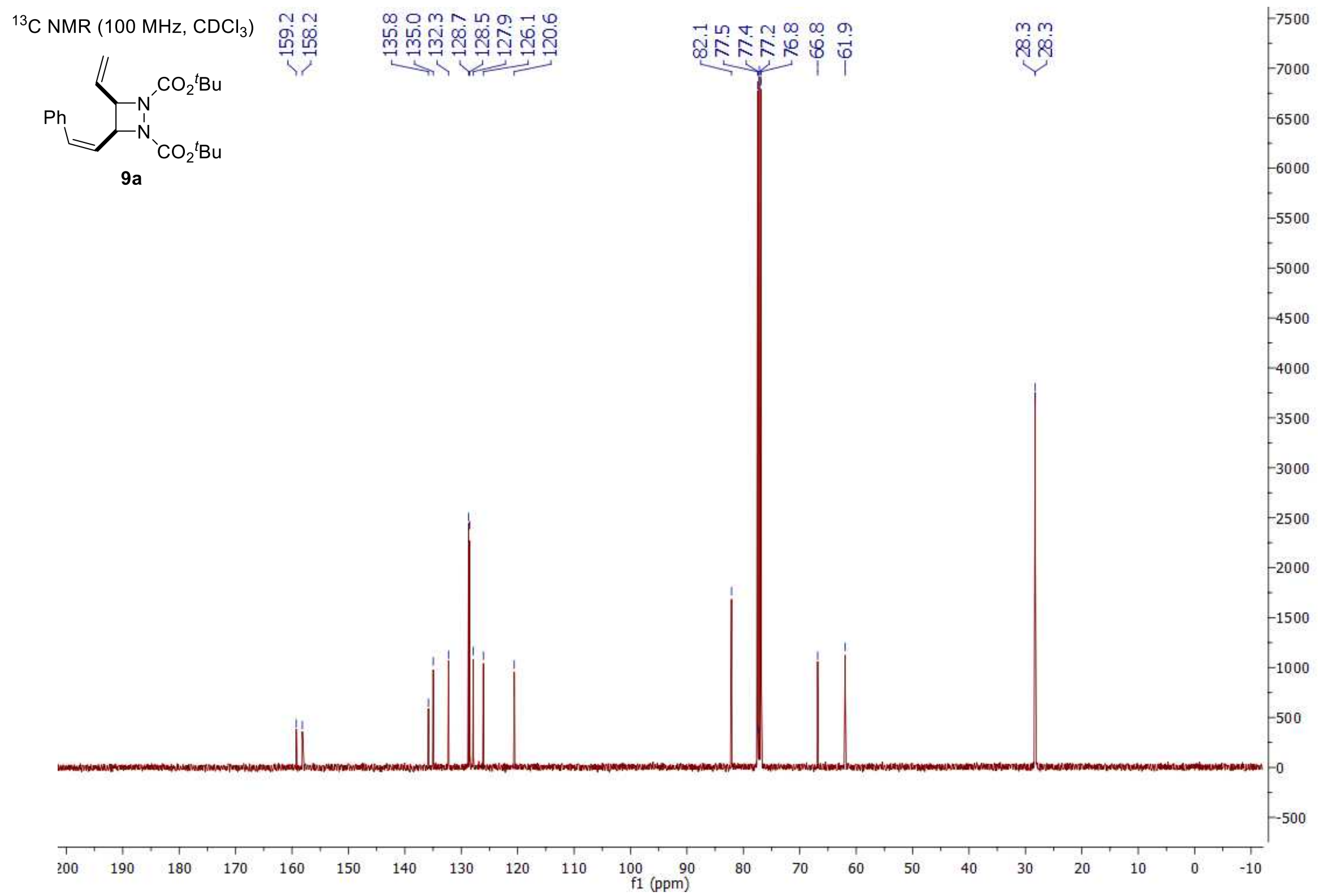




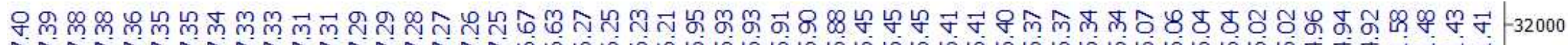

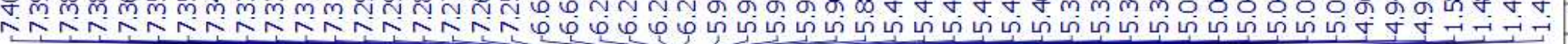

${ }^{1} \mathrm{H}$ NMR $\left(400 \mathrm{MHz}, \mathrm{CDCl}_{3}\right)$
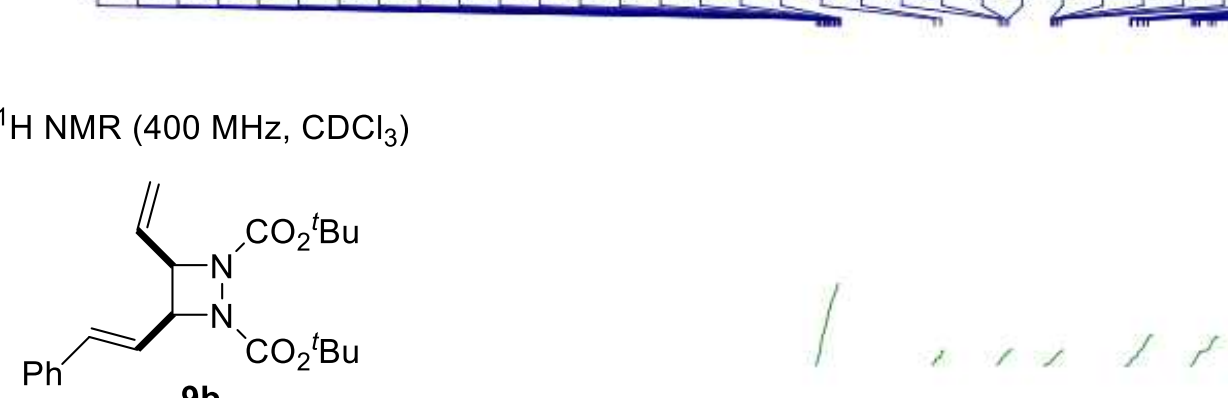

$9 b$

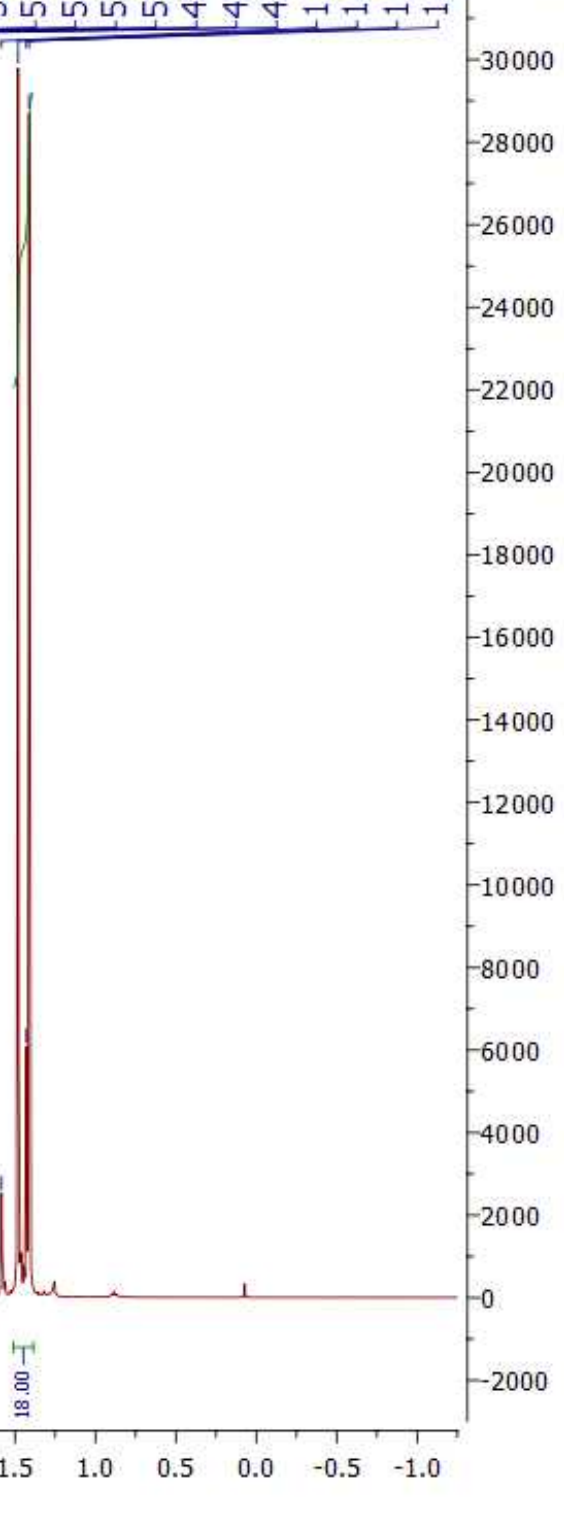




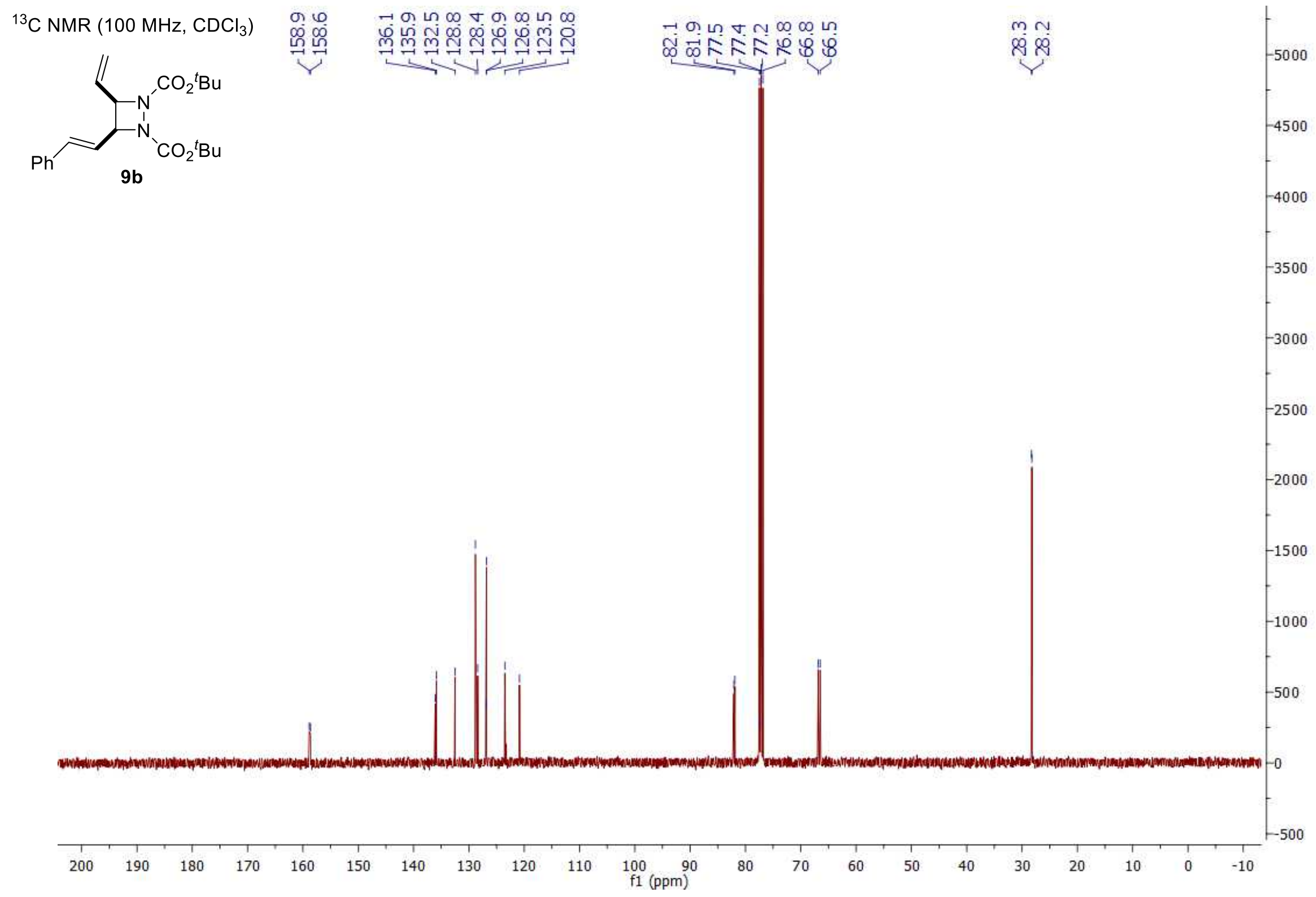




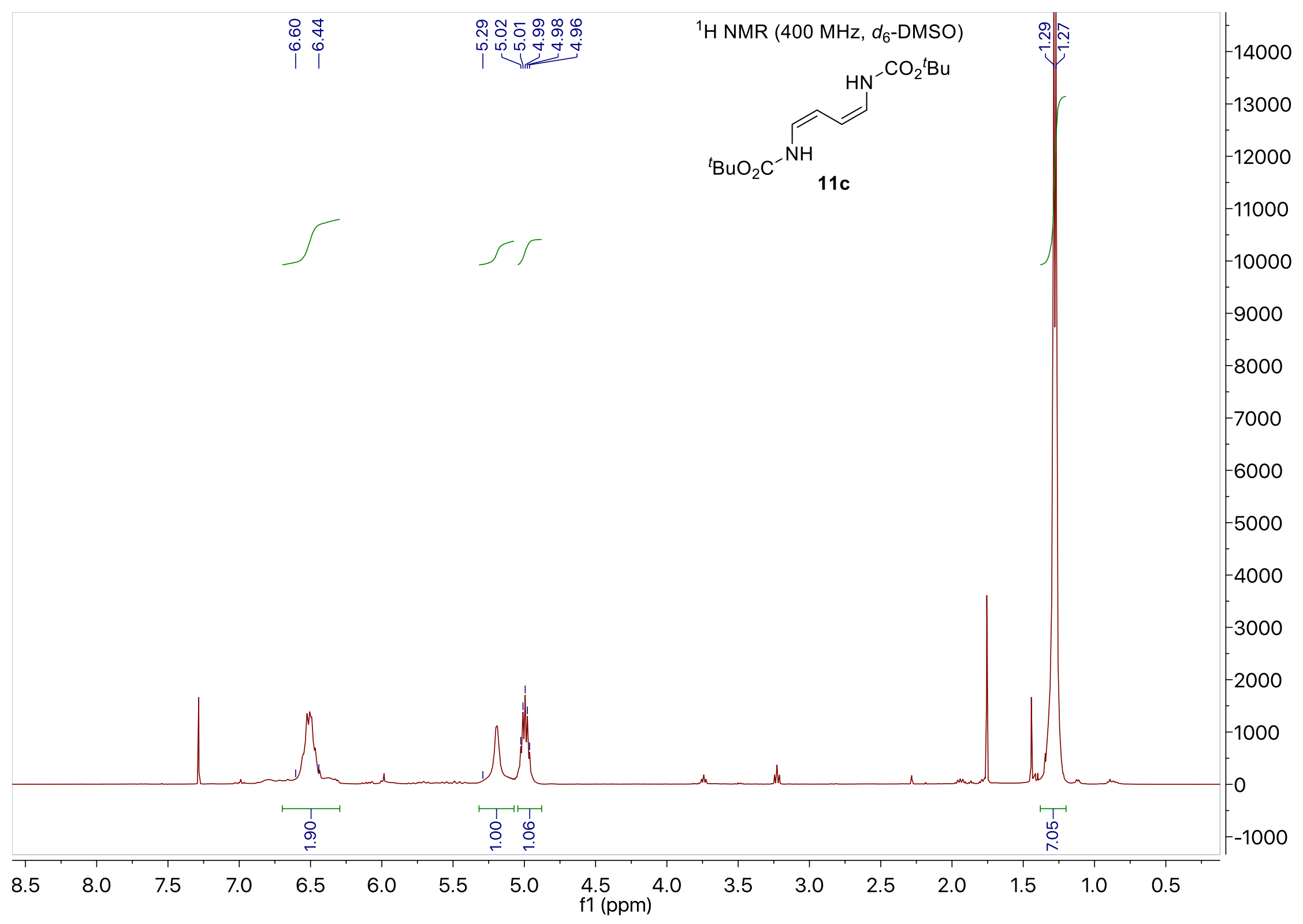




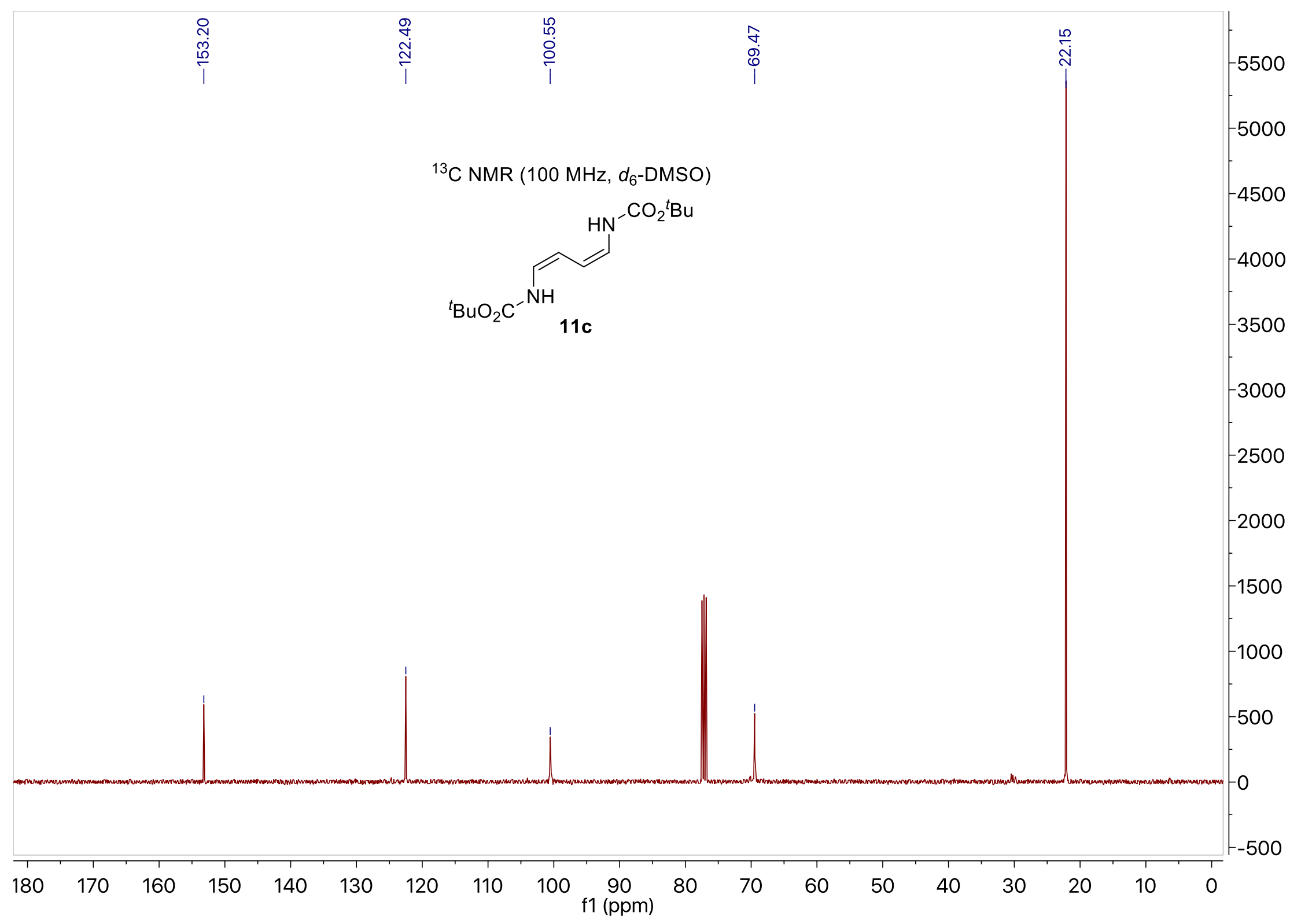




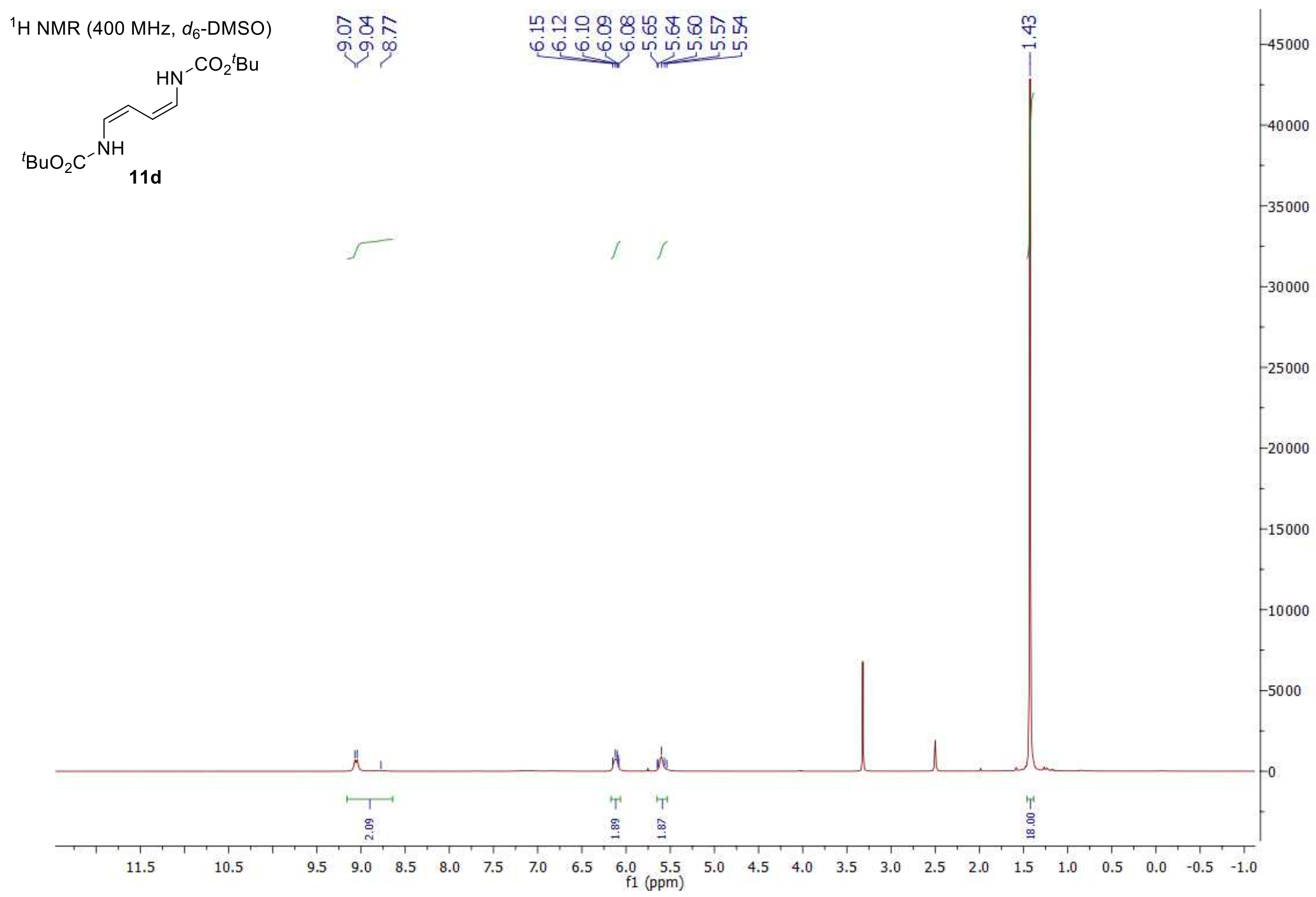




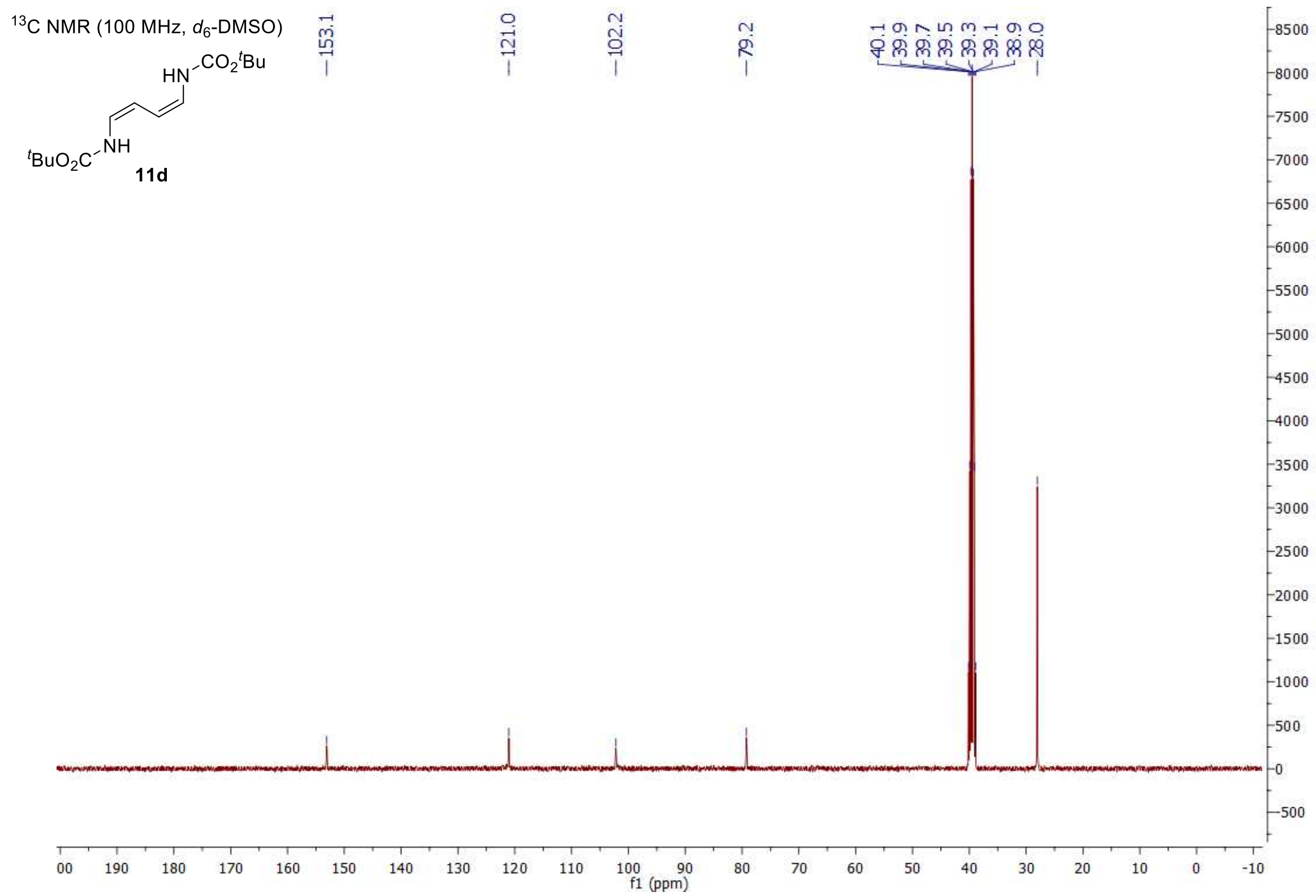




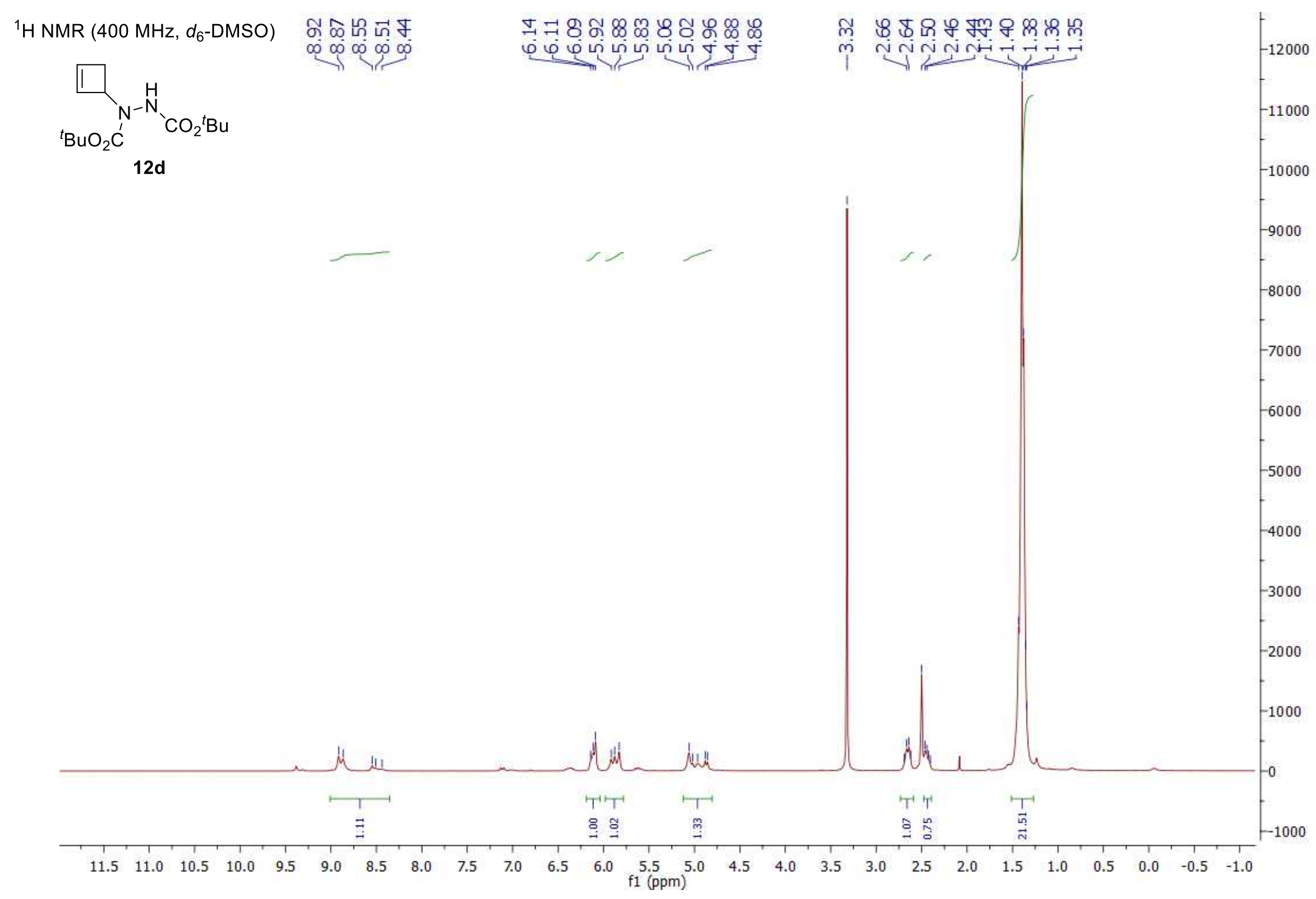




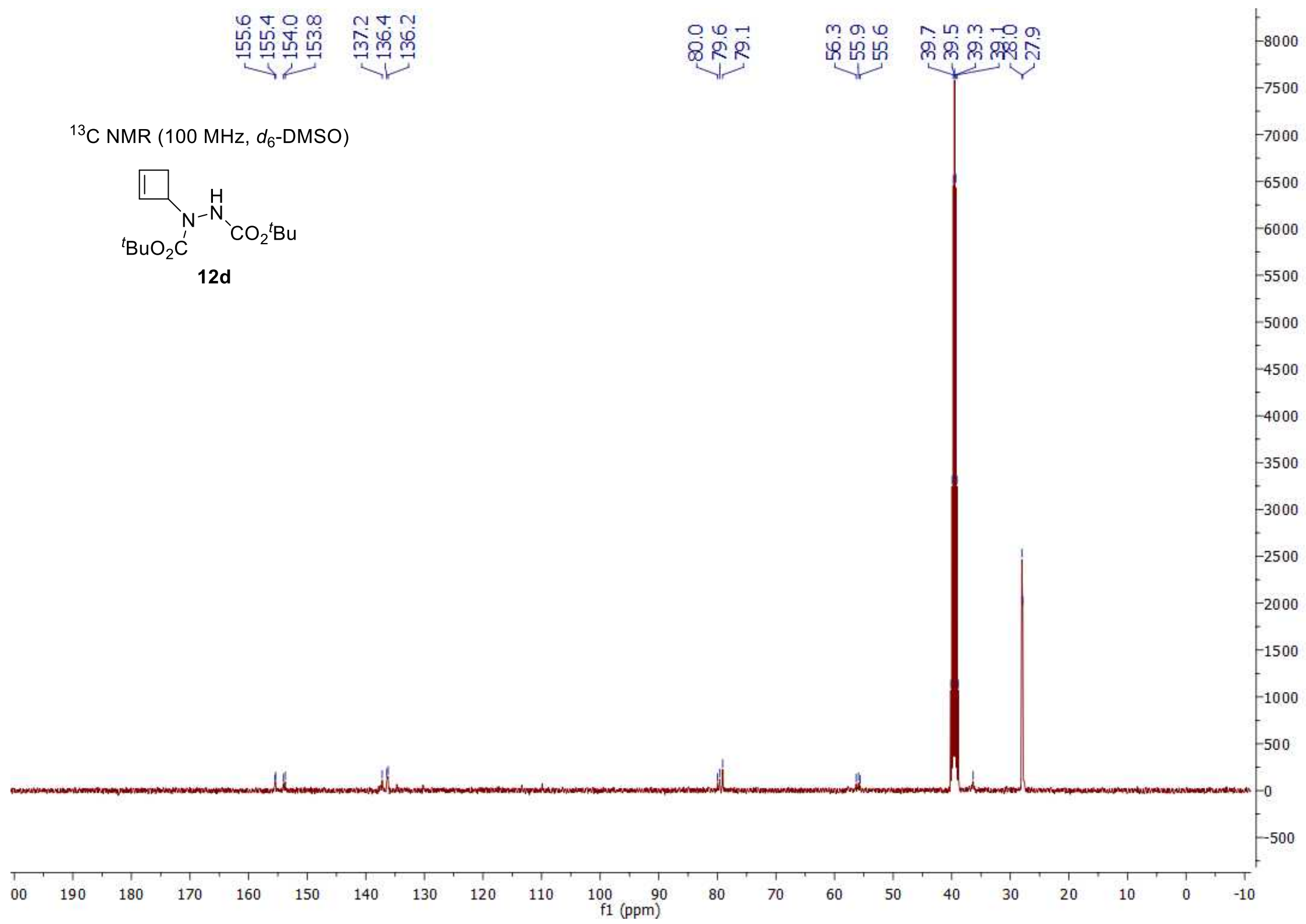


莳品

बं

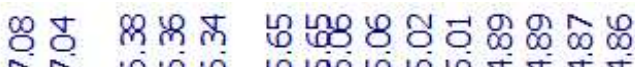

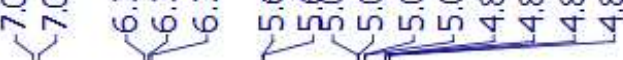

${ }^{1} \mathrm{H}$ NMR (400 MHz, $d_{6}$-DMSO)

$\leadsto \mathrm{N}_{\mathrm{CO}_{2}{ }^{t} \mathrm{Bu}}^{\mathrm{N}} \mathrm{CO}_{2}{ }^{t} \mathrm{Bu}$

$13 d$
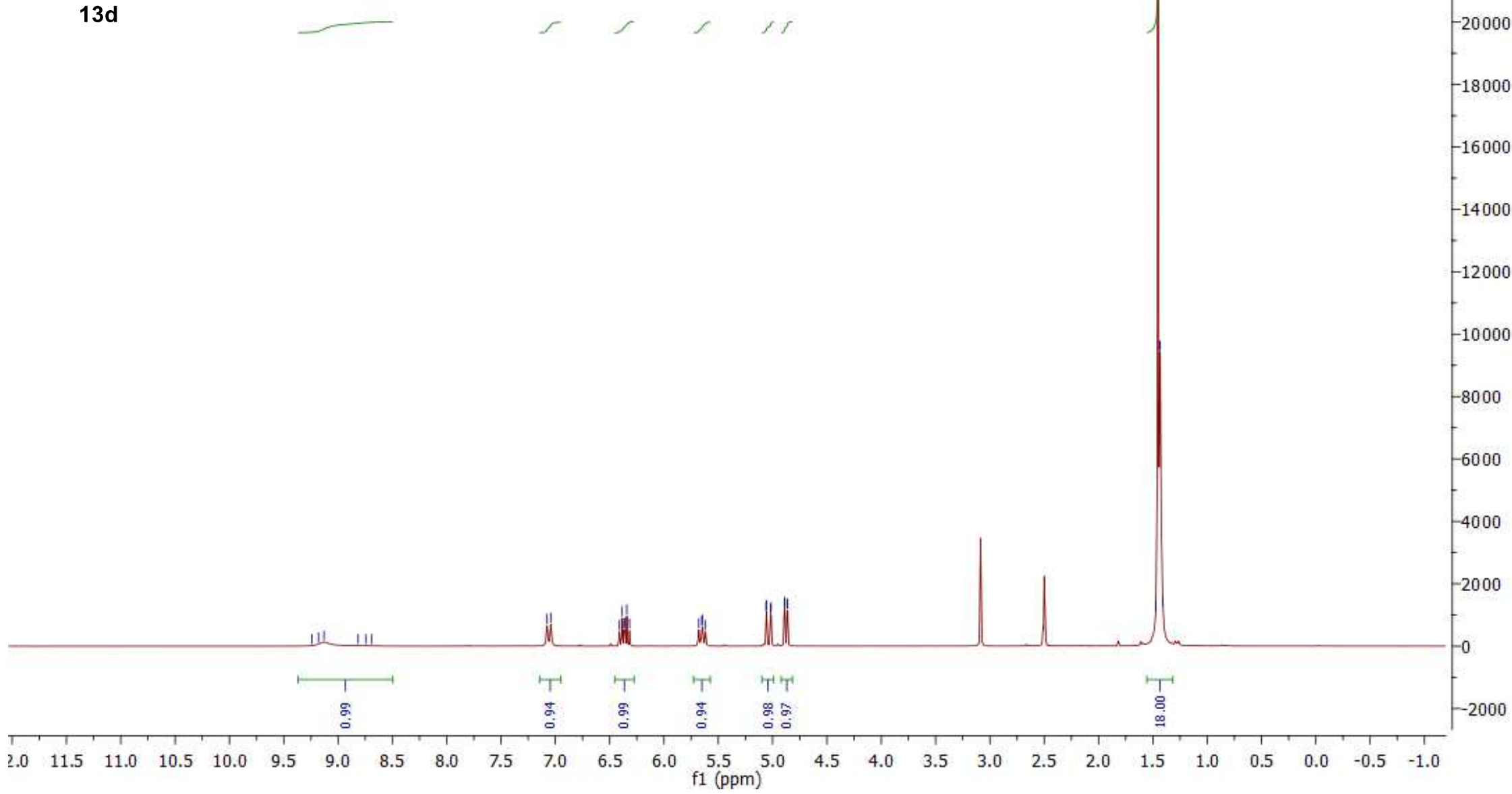


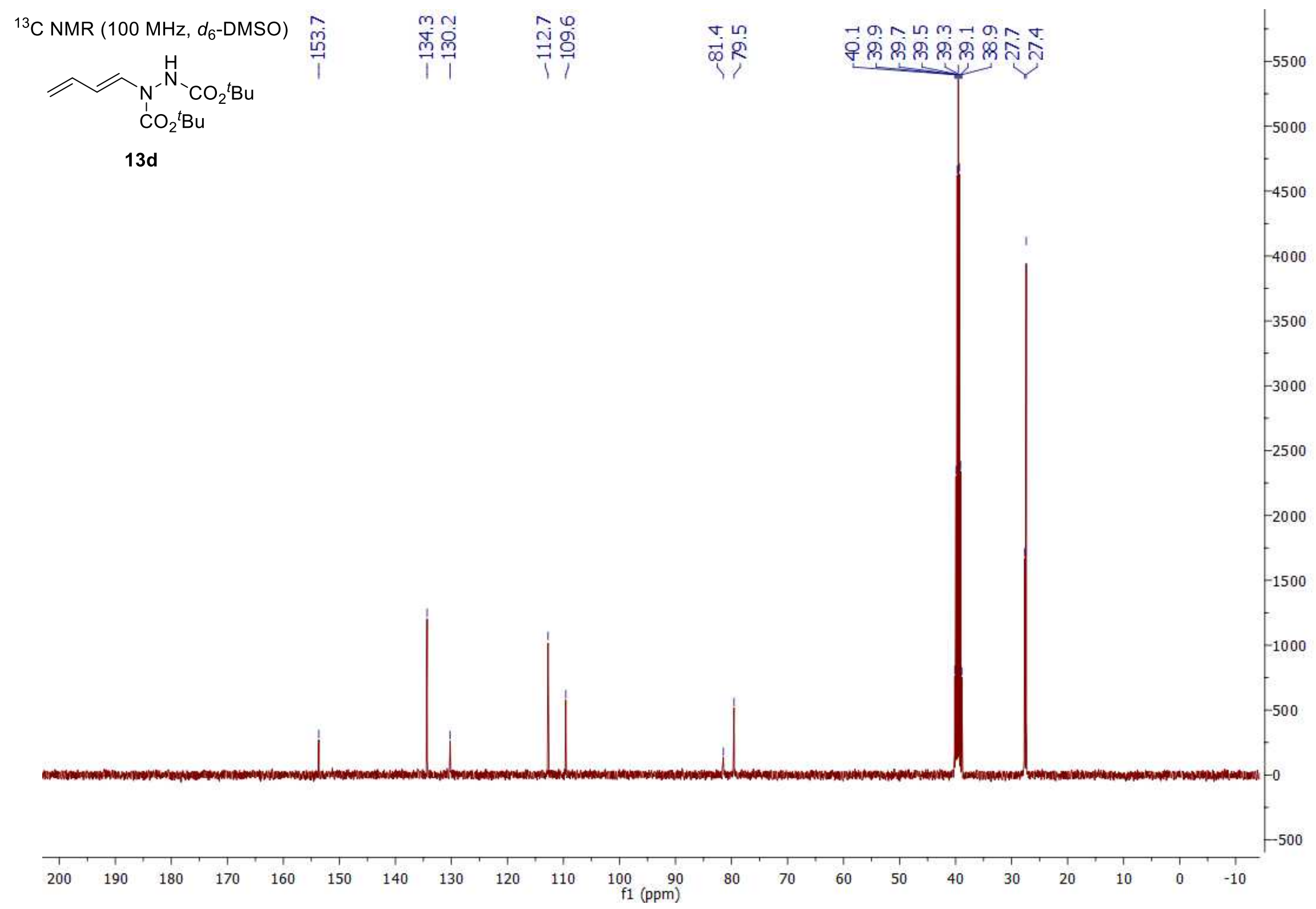




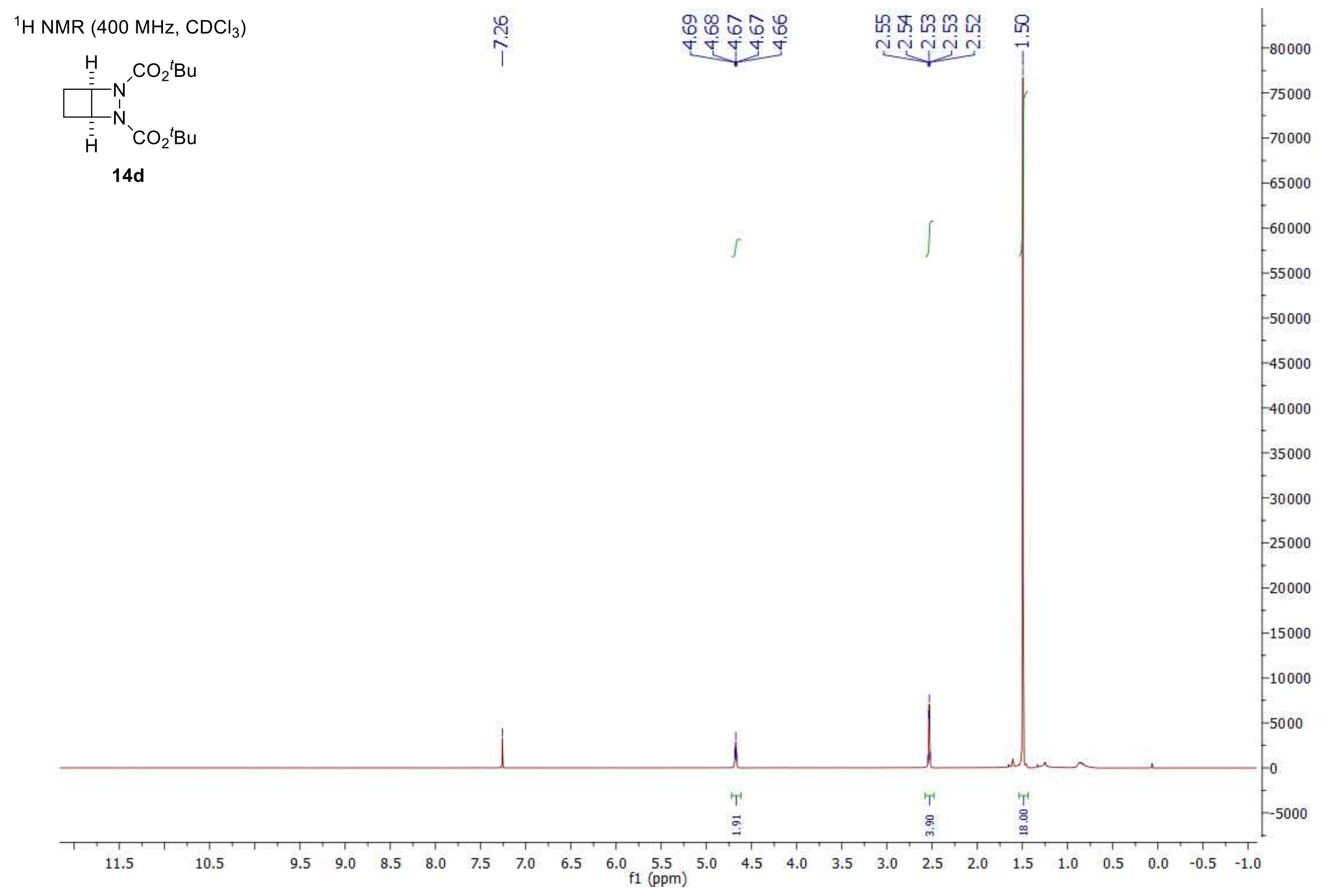




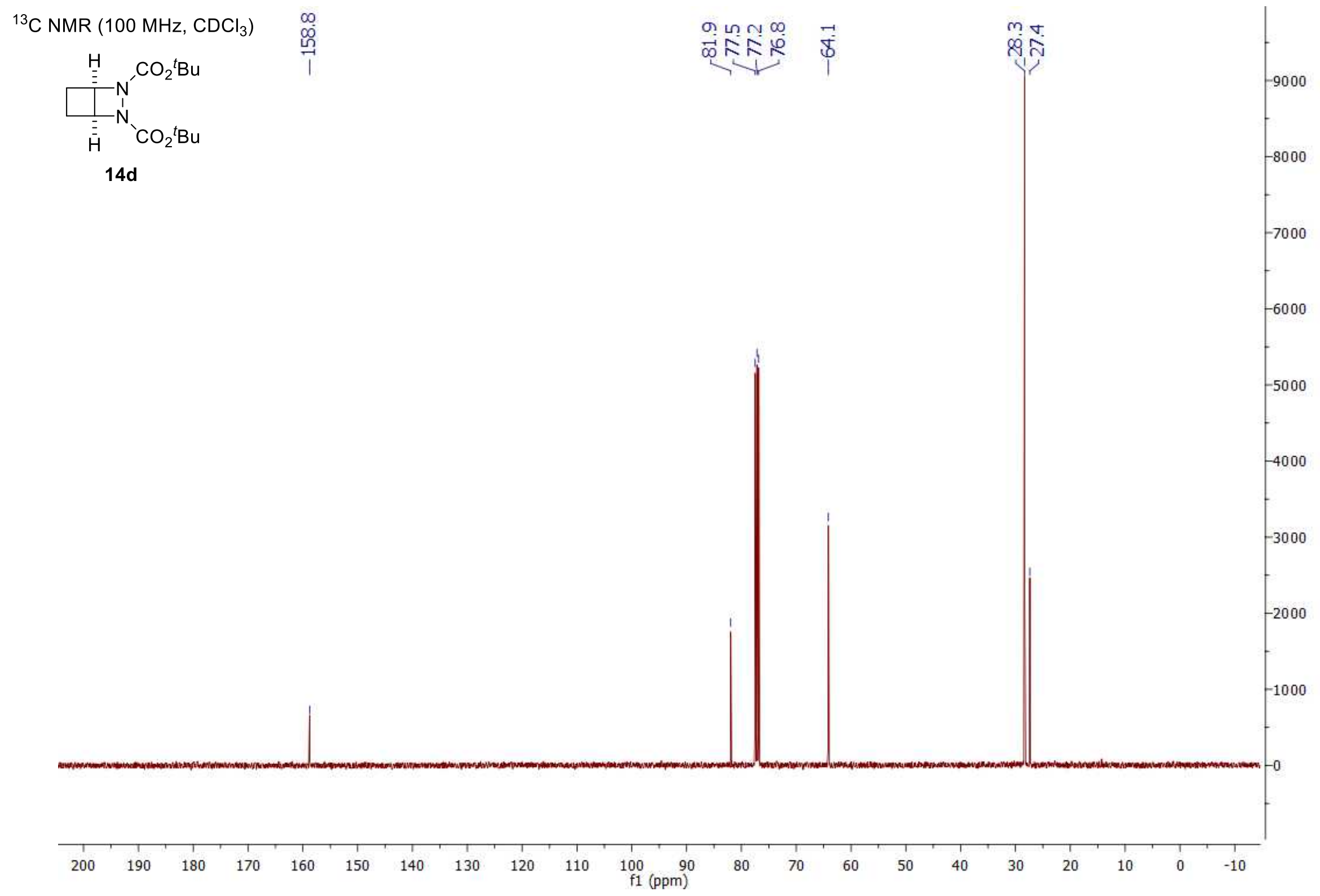




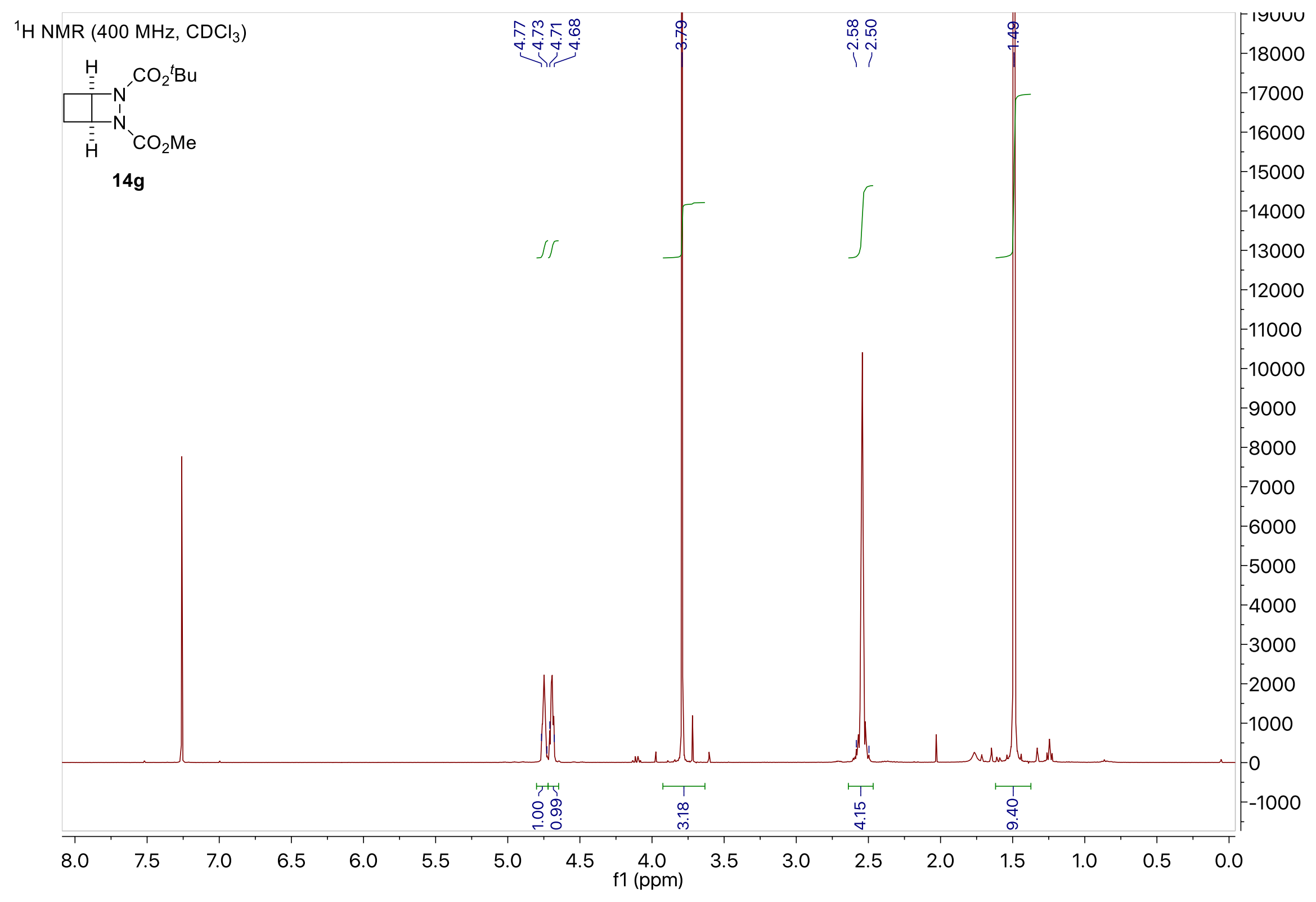




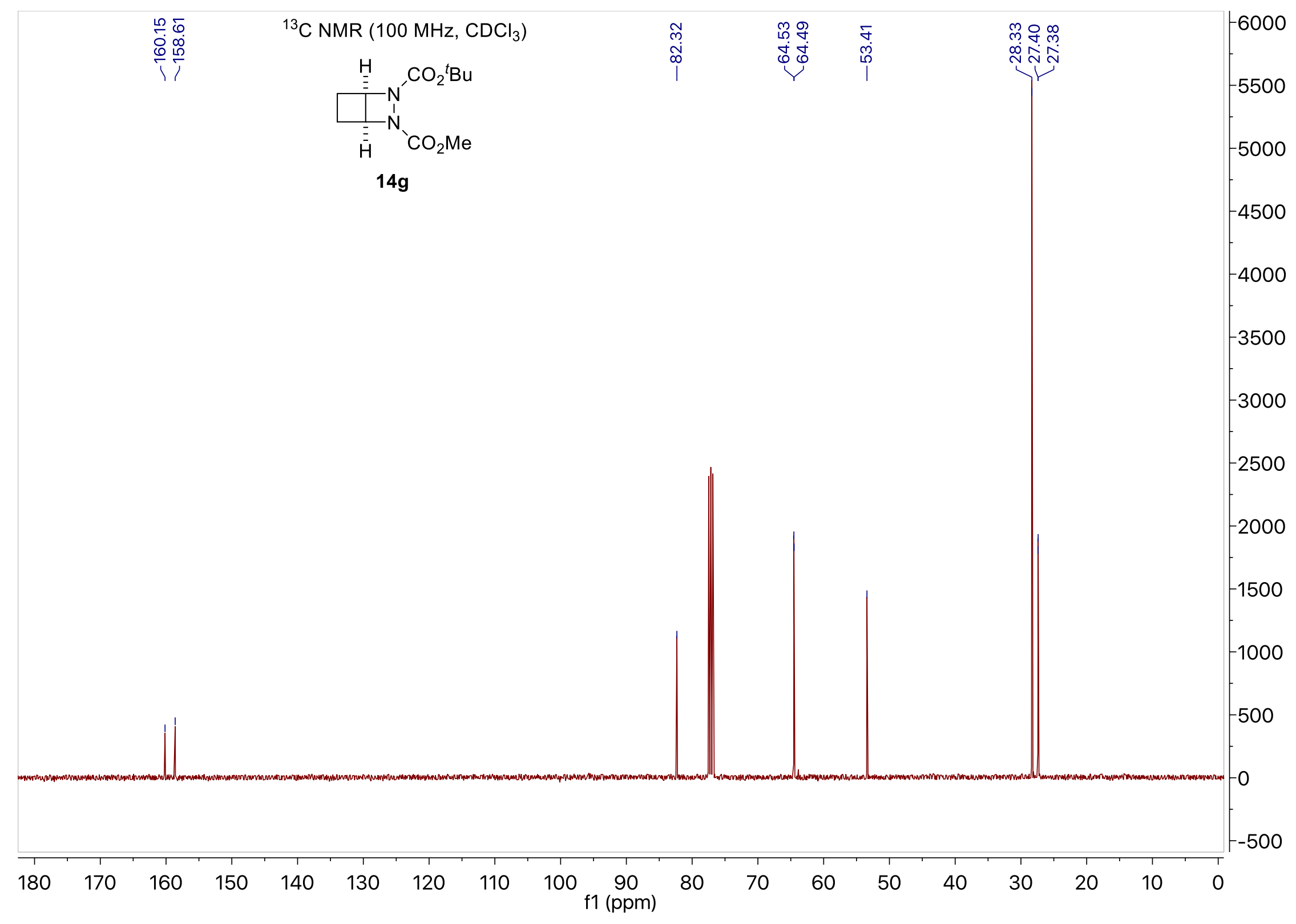

S68 


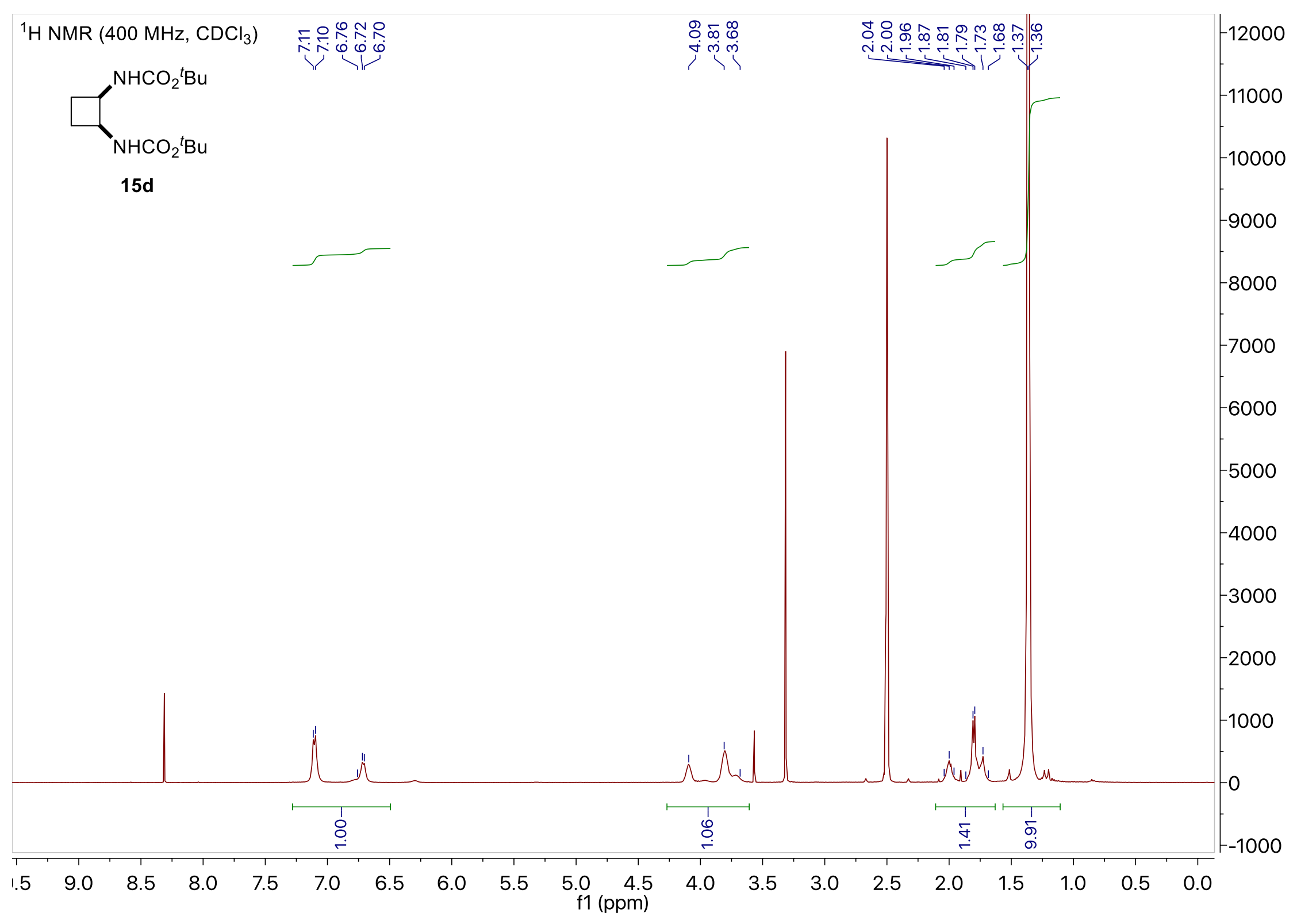




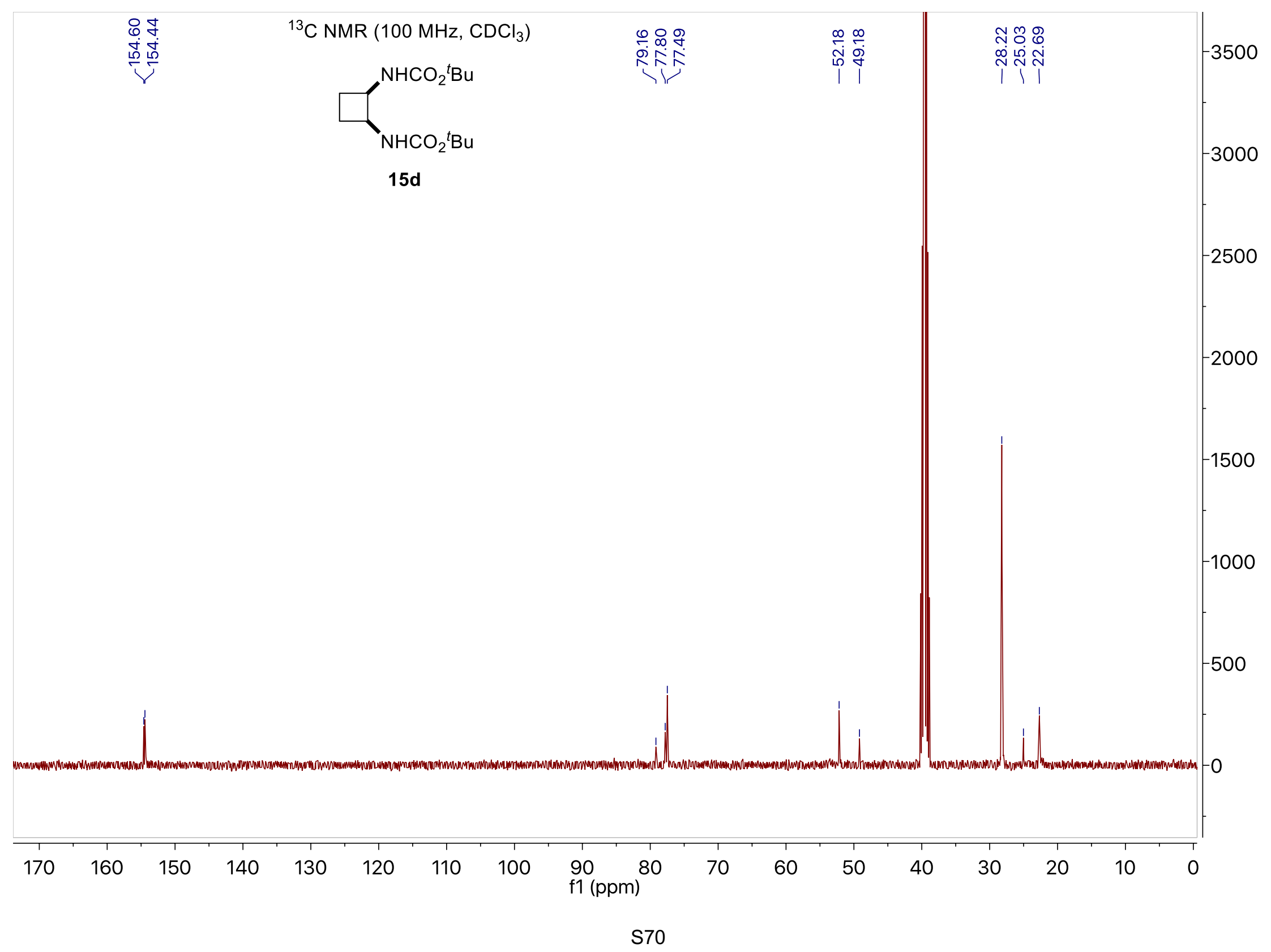




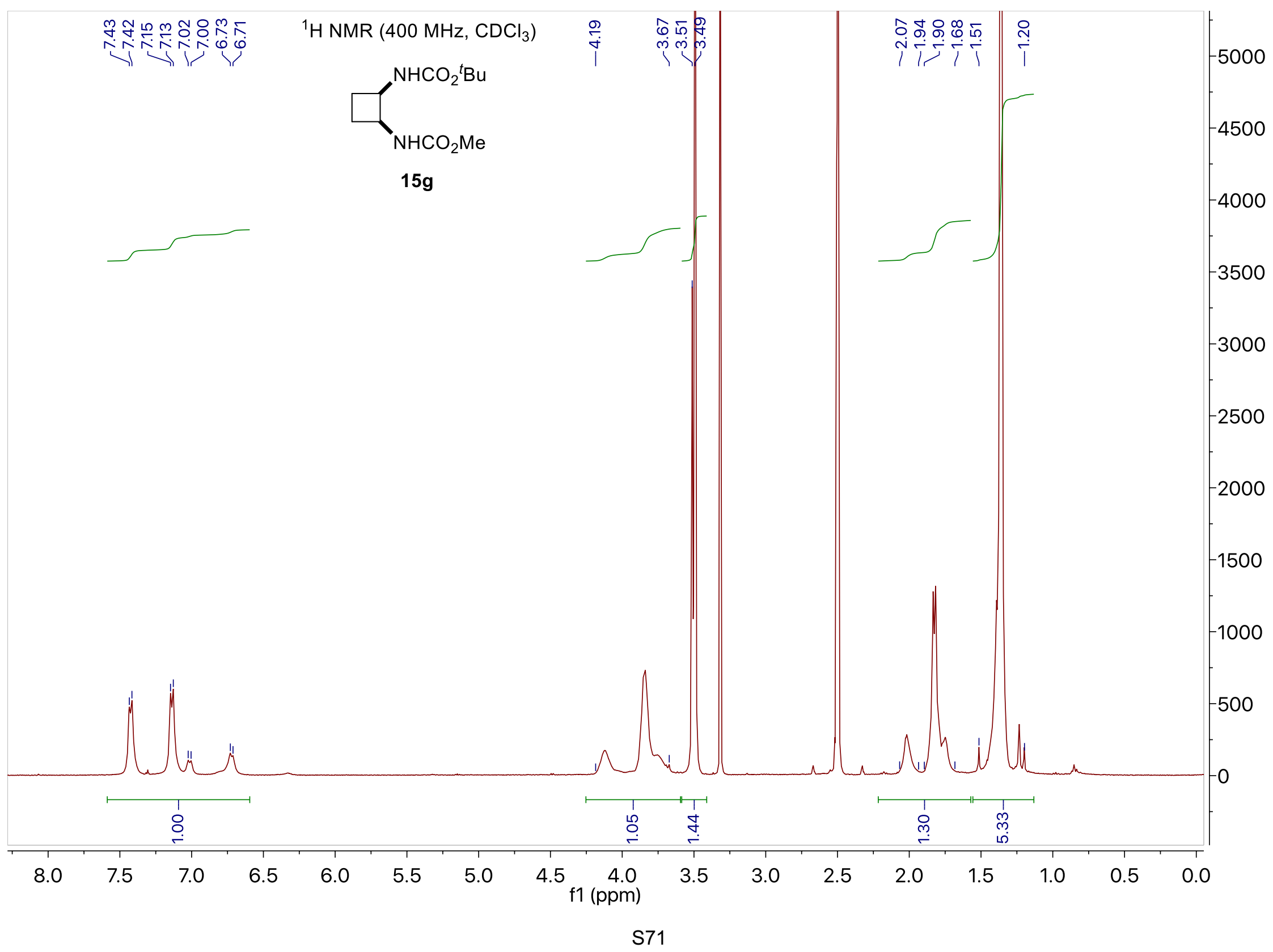




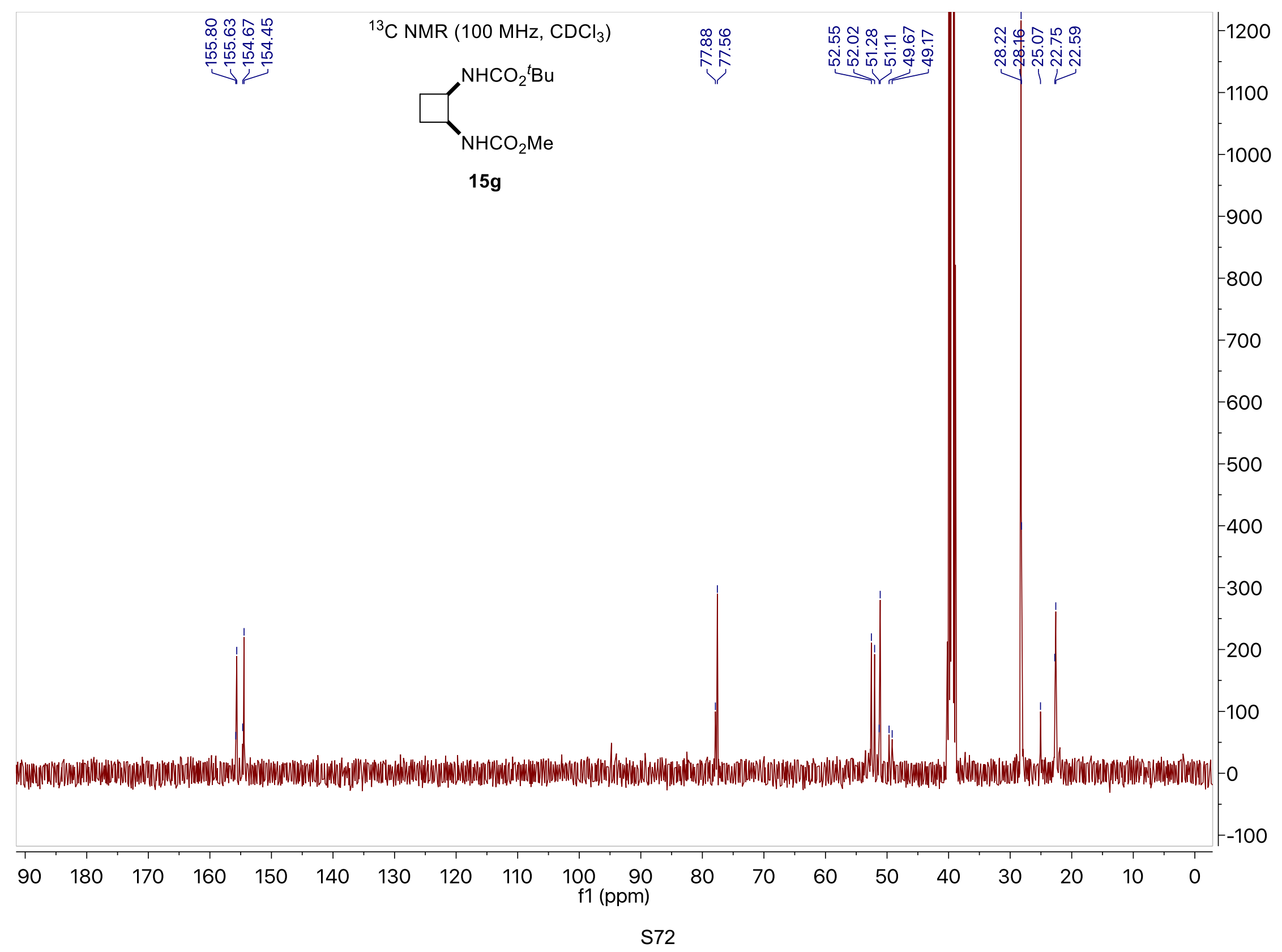




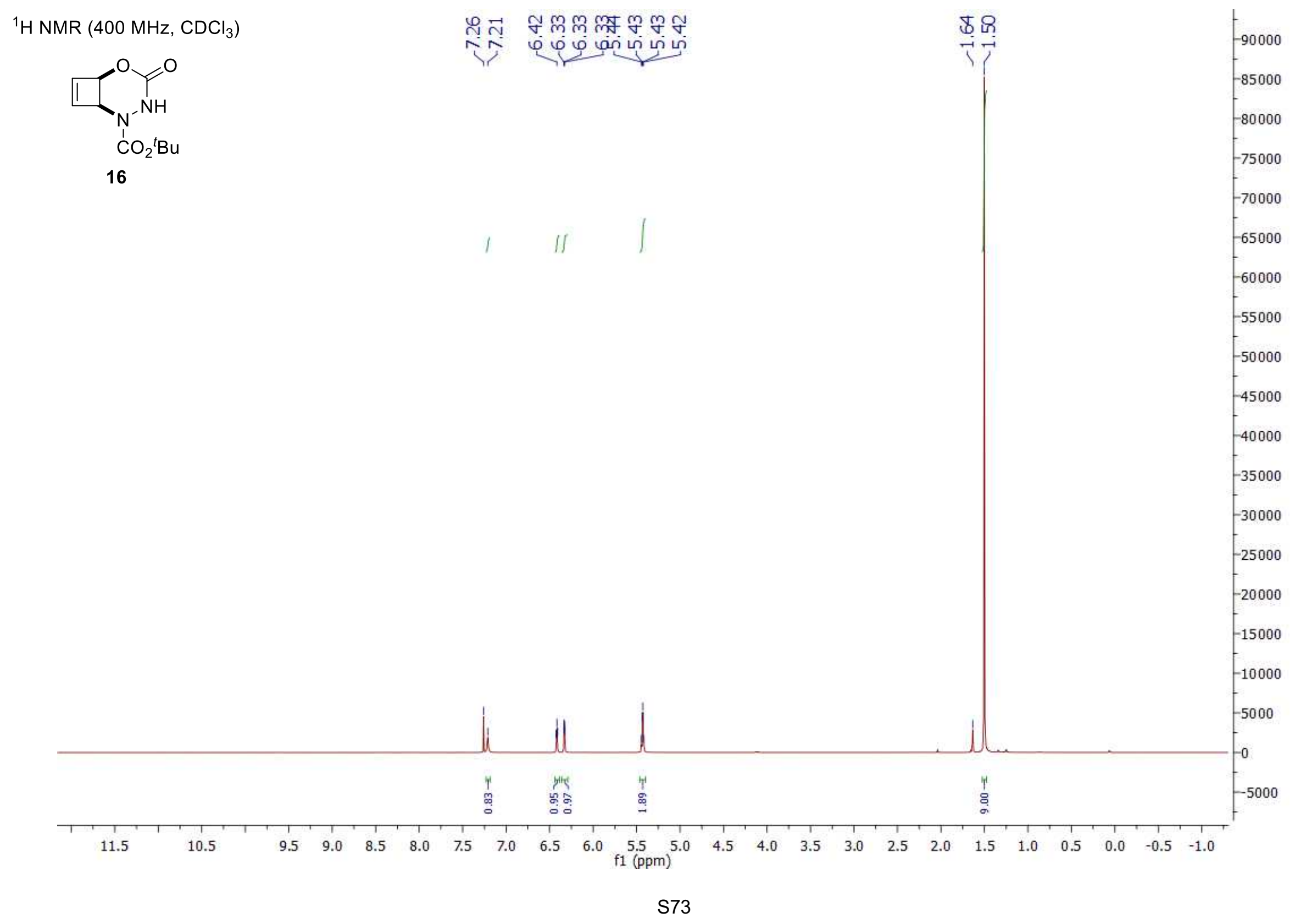




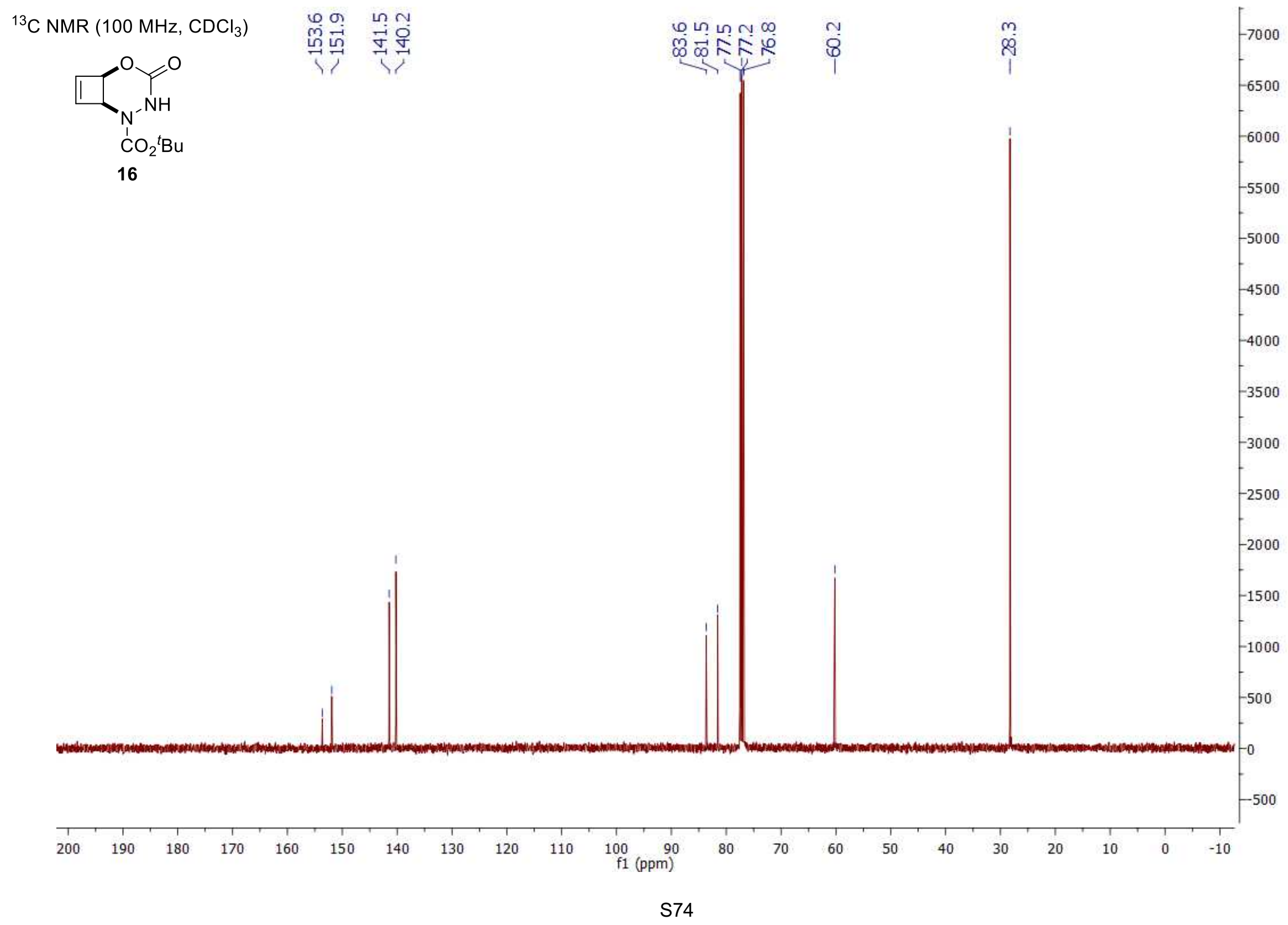




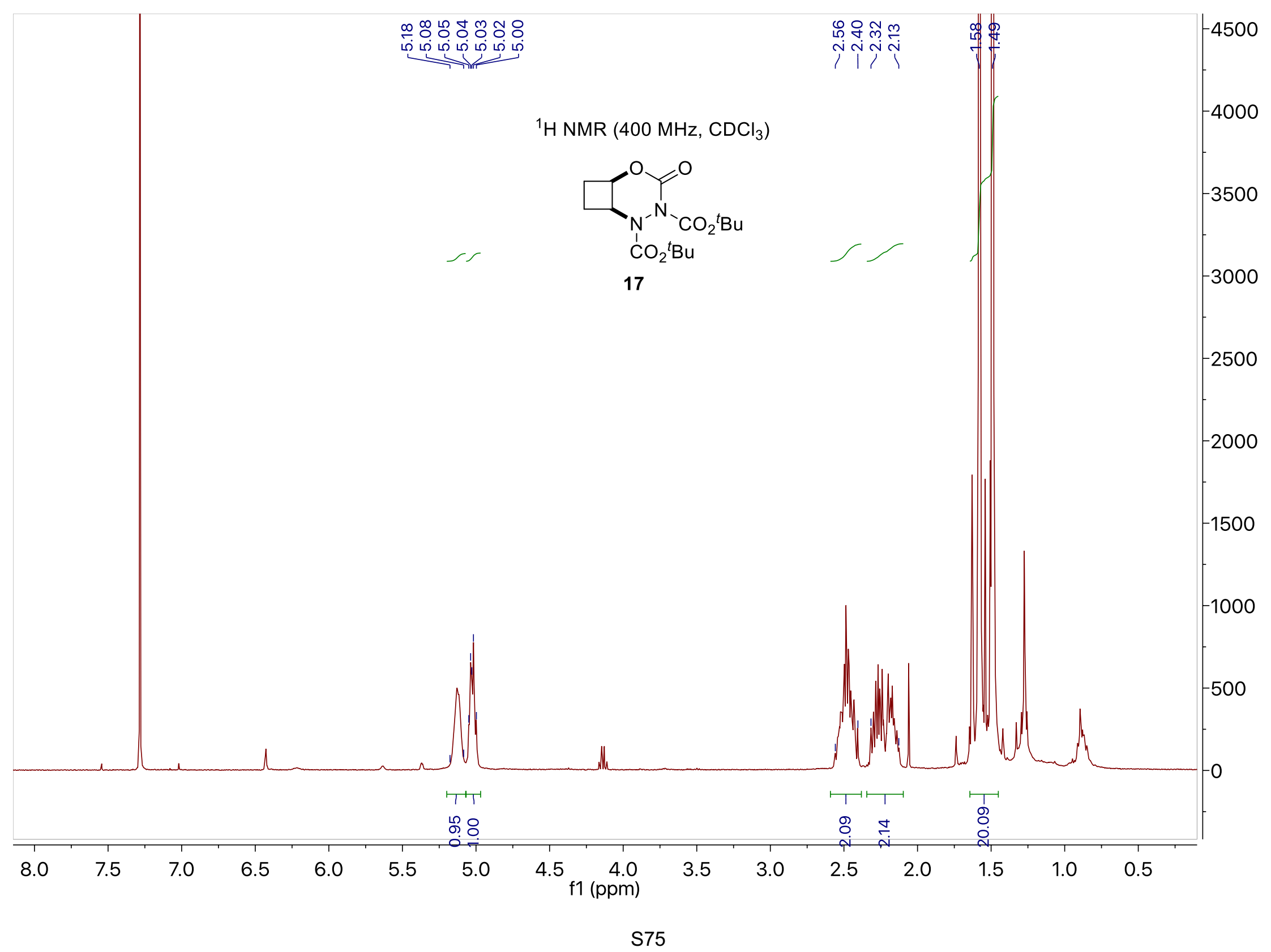




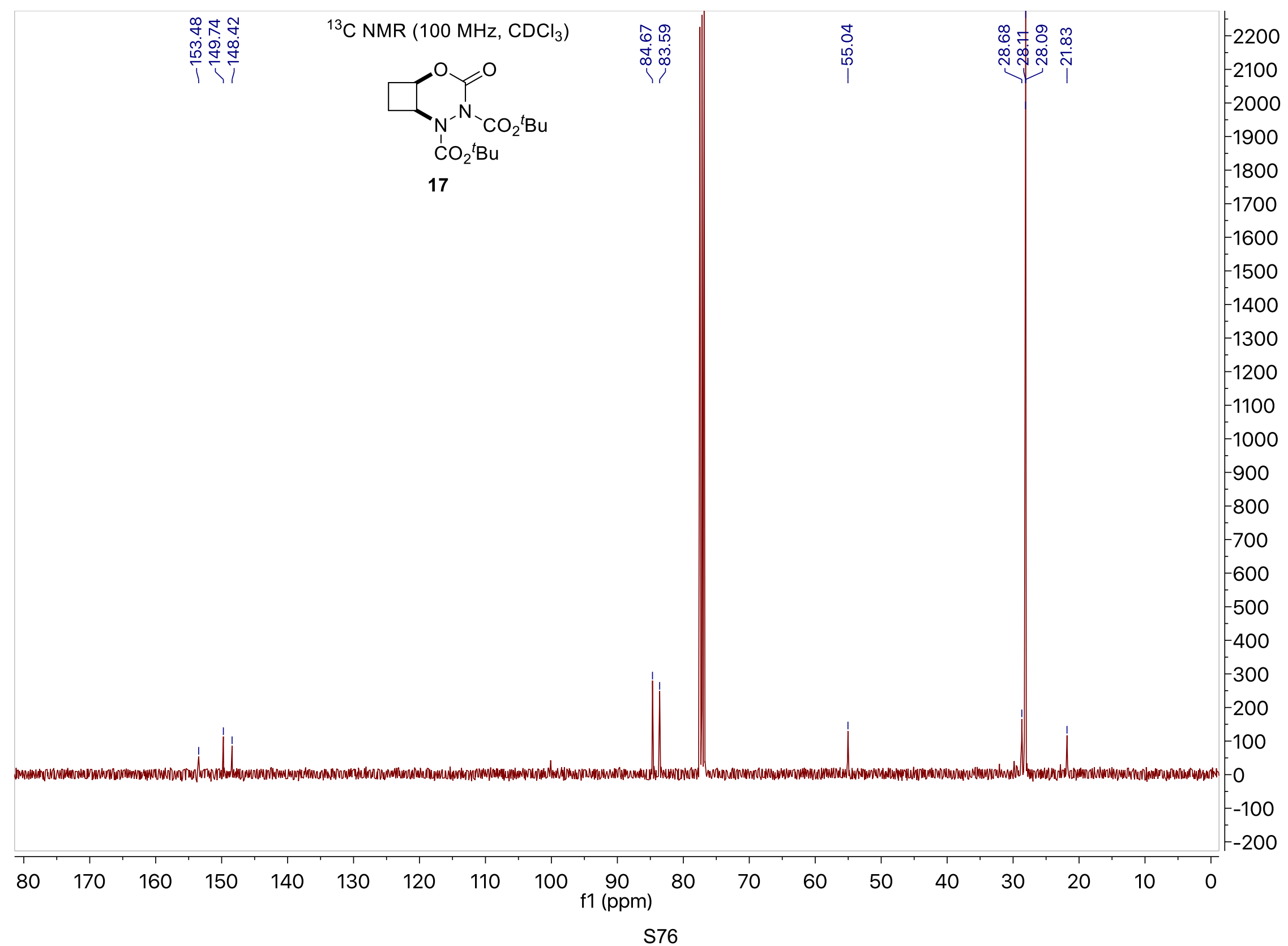




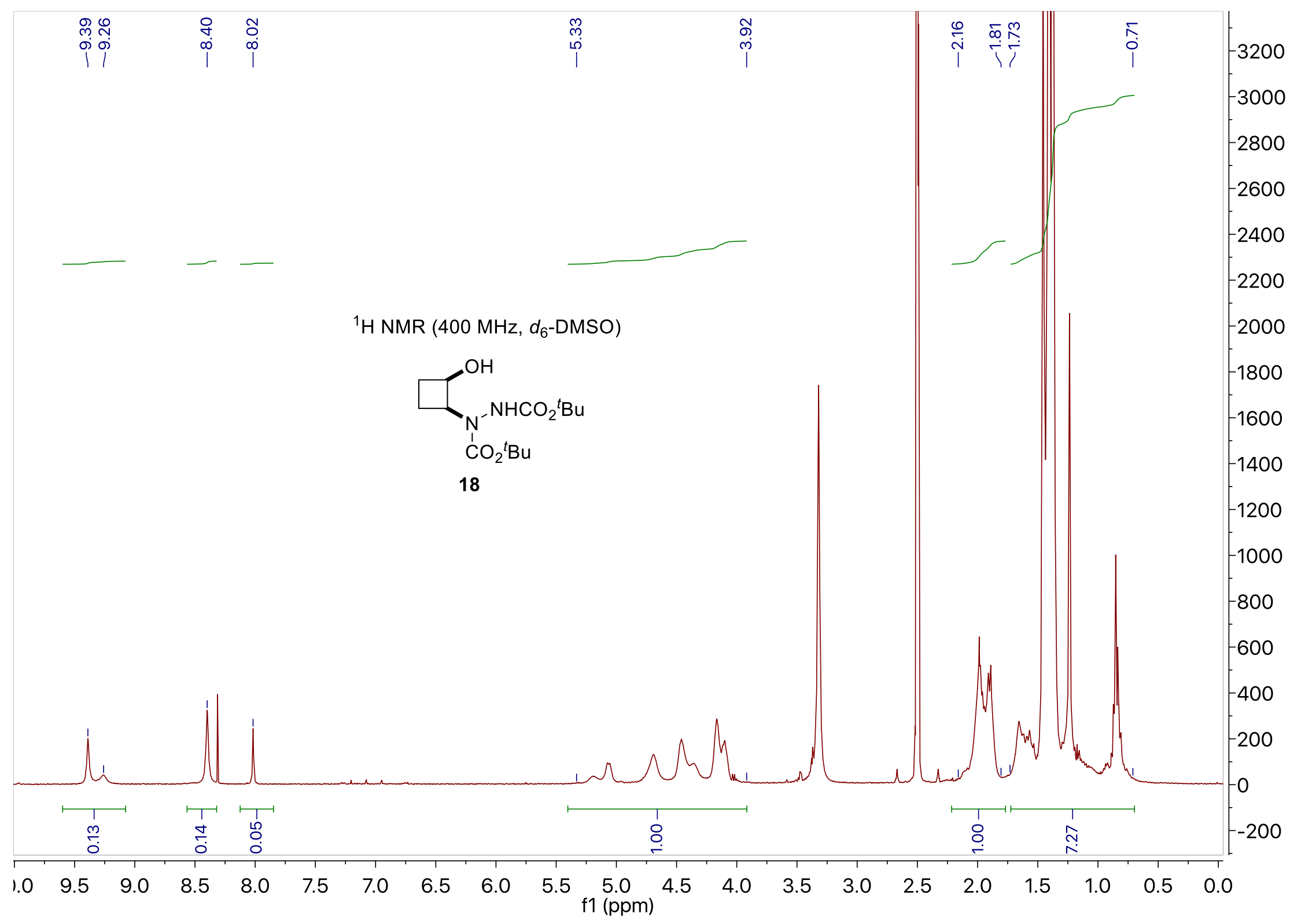




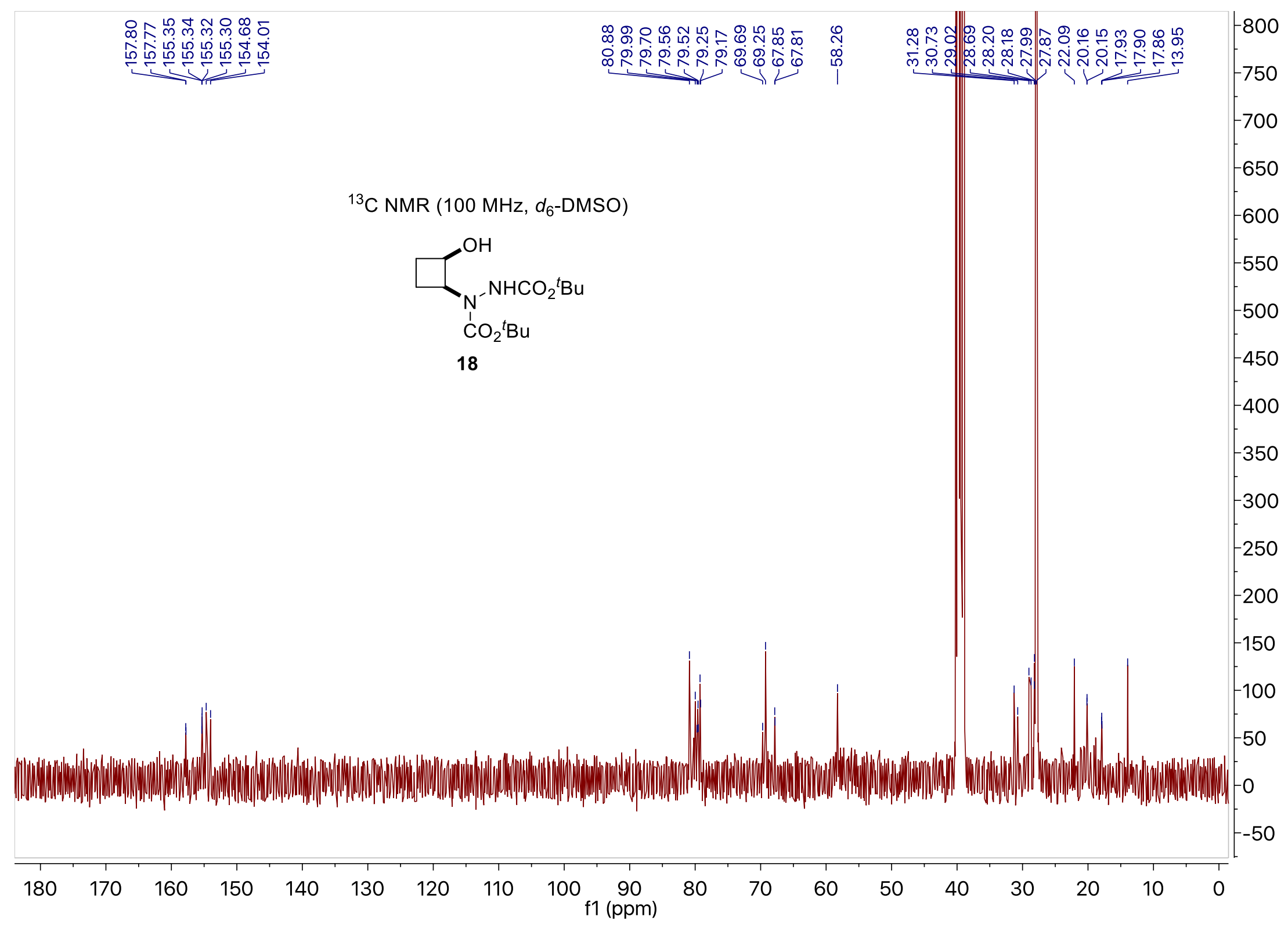




\section{X-Ray Diffraction Data for $2 d, 11 d, 16$ and 18}

X-Ray Crystal Structure Data for 2d (CCDC 1941740)
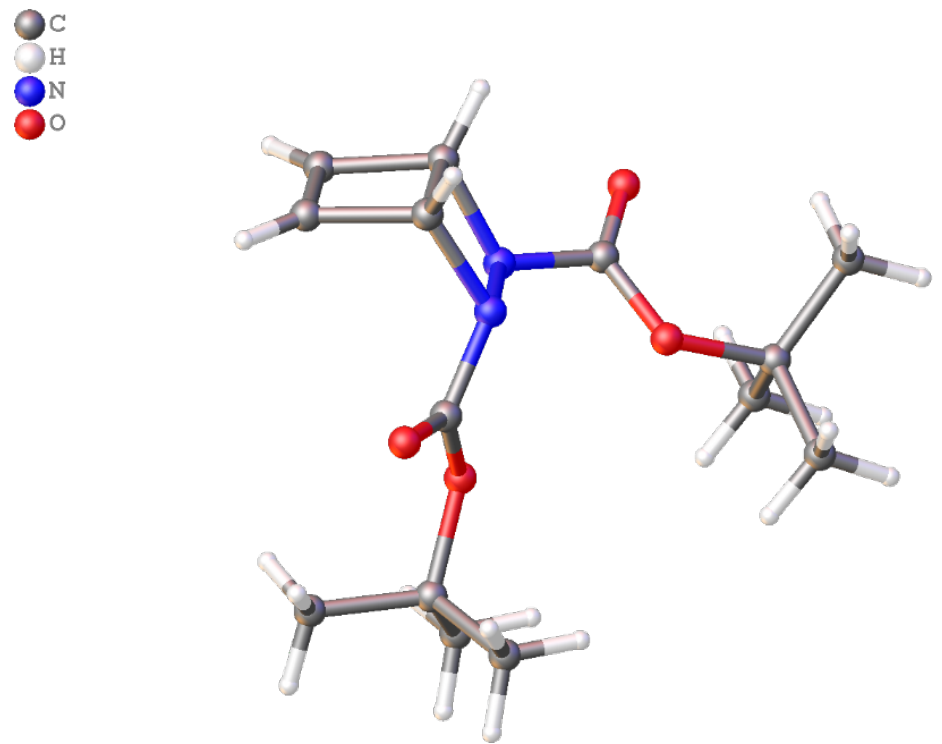

Identification code

Empirical formula

Formula weight

Temperature/K

Crystal system

Space group

$\mathrm{a} / \AA ̊$

$\mathrm{b} / \AA ̊$

$c / \AA$

$\alpha /{ }^{\circ}$

$\beta /^{\circ}$

$\mathrm{Y} /{ }^{\circ}$

Volume $/ \AA^{3}$

Z

$\rho_{\text {calc }} \mathrm{g} / \mathrm{cm}^{3}$

$\mu / \mathrm{mm}^{-1}$

$F(000)$
SC107

$\mathrm{C}_{14} \mathrm{H}_{22} \mathrm{~N}_{2} \mathrm{O}_{4}$

282.33

99.99(10)

monoclinic

$\mathrm{P} 2{ }_{1} / \mathrm{c}$

8.95843(13)

19.3911(2)

9.66742(12)

90

114.1195(17)

90

1532.75(4)

4

1.223

0.740

608.0 
Crystal size $/ \mathrm{mm}^{3}$

Radiation

$2 \Theta$ range for data collection/ ${ }^{\circ} 9.122$ to 153.564

Index ranges

Reflections collected

Independent reflections

Data/restraints/parameters

Goodness-of-fit on $\mathrm{F}^{2}$

1.027

Final $R$ indexes $[l>=2 \sigma(I)] \quad R_{1}=0.0369, w R_{2}=0.0913$

Final $R$ indexes [all data] $\quad R_{1}=0.0374, w_{2}=0.0918$

Largest diff. peak/hole / e $\AA^{-3}$ 0.25/-0.24

X-Ray Crystal Structure Data for 11b (CCDC 1941741)

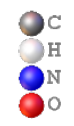

Identification code

SC117

Empirical formula

$\mathrm{C}_{14} \mathrm{H}_{24} \mathrm{~N}_{2} \mathrm{O}_{4}$

Formula weight

284.35

Temperature/K

99.9(2)

Crystal system

monoclinic

Space group

$\mathrm{P} 21 / \mathrm{n}$

$\mathrm{a} / \AA \AA$

$5.23750(9)$

$\mathrm{b} / \AA ̊$

$8.83307(15)$ 


\begin{tabular}{|c|c|}
\hline$c / \AA ̊$ & $16.5180(3)$ \\
\hline$\alpha /{ }^{\circ}$ & 90 \\
\hline$\beta /^{\circ}$ & $92.6082(17)$ \\
\hline $\mathrm{Y}^{\circ}$ & 90 \\
\hline Volume/ $\AA^{3}$ & $763.39(2)$ \\
\hline Z & 2 \\
\hline$\rho_{\text {calc }} \mathrm{g} / \mathrm{cm}^{3}$ & 1.237 \\
\hline$\mu / \mathrm{mm}^{-1}$ & 0.743 \\
\hline$F(000)$ & 308.0 \\
\hline Crystal size $/ \mathrm{mm}^{3}$ & $0.109 \times 0.098 \times 0.082$ \\
\hline Radiation & CuKa $(\lambda=1.54184)$ \\
\hline \multicolumn{2}{|c|}{$2 \Theta$ range for data collection $/{ }^{\circ} 10.724$ to 153} \\
\hline Index ranges & $-6 \leq h \leq 6,-11 \leq k \leq 11,-17 \leq \mathrm{I} \leq 20$ \\
\hline Reflections collected & 9639 \\
\hline Independent reflections & $1589\left[R_{\text {int }}=0.0415, R_{\text {sigma }}=0.0201\right]$ \\
\hline Data/restraints/parameters & $1589 / 0 / 94$ \\
\hline Goodness-of-fit on $\mathrm{F}^{2}$ & 1.058 \\
\hline Final $R$ indexes $[I>=2 \sigma(I)]$ & $R_{1}=0.0369, w R_{2}=0.0961$ \\
\hline Final $\mathrm{R}$ indexes [all data] & $\mathrm{R}_{1}=0.0386, \mathrm{wR}_{2}=0.0977$ \\
\hline
\end{tabular}

X-Ray Crystal Structure Data for 16 (CCDC 1941742)

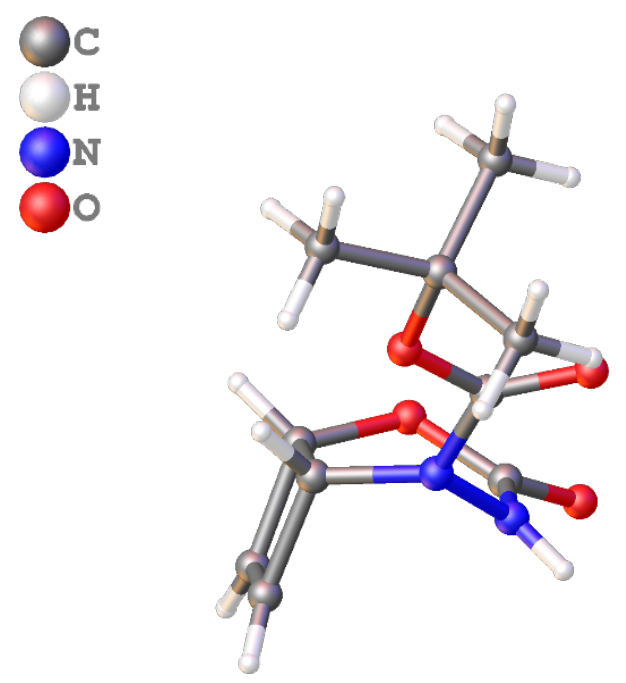




\begin{tabular}{|c|c|}
\hline Identification code & SC108 \\
\hline Empirical formula & $\mathrm{C}_{10} \mathrm{H}_{14} \mathrm{~N}_{2} \mathrm{O}_{4}$ \\
\hline Formula weight & 226.23 \\
\hline Temperature/K & $100.2(4)$ \\
\hline Crystal system & monoclinic \\
\hline Space group & $\mathrm{P} 2{ }_{1} / \mathrm{c}$ \\
\hline $\mathrm{a} / \AA \AA$ & $10.3330(6)$ \\
\hline $\mathrm{b} / \AA \AA$ & $9.5174(5)$ \\
\hline$c / \AA$ & $11.5317(7)$ \\
\hline$\alpha /^{\circ}$ & 90 \\
\hline$\beta /^{\circ}$ & $102.715(7)$ \\
\hline $\mathrm{Y} /{ }^{\circ}$ & 90 \\
\hline Volume/ $\AA^{3}$ & $1106.26(11)$ \\
\hline Z & 4 \\
\hline$\rho_{\text {calcg }} / \mathrm{cm}^{3}$ & 1.358 \\
\hline$\mu / \mathrm{mm}^{-1}$ & 0.893 \\
\hline$F(000)$ & 480.0 \\
\hline Crystal size $/ \mathrm{mm}^{3}$ & $0.171 \times 0.142 \times 0.07$ \\
\hline Radiation & CuKa $(\lambda=1.54184)$ \\
\hline \multicolumn{2}{|c|}{$2 \Theta$ range for data collection $/{ }^{\circ} 8.772$ to 152.362} \\
\hline Index ranges & $-12 \leq \mathrm{h} \leq 12,-11 \leq \mathrm{k} \leq 11,-14 \leq \mathrm{I} \leq 8$ \\
\hline Reflections collected & 6952 \\
\hline Independent reflections & $2281\left[R_{\text {int }}=0.0313, R_{\text {sigma }}=0.0277\right]$ \\
\hline Data/restraints/parameters & $2281 / 0 / 148$ \\
\hline Goodness-of-fit on $\mathrm{F}^{2}$ & 1.056 \\
\hline Final $R$ indexes $[l>=2 \sigma(I)]$ & $\mathrm{R}_{1}=0.0545, \mathrm{wR}_{2}=0.1455$ \\
\hline Final $R$ indexes [all data] & $\mathrm{R}_{1}=0.0604, w R_{2}=0.1504$ \\
\hline Largest diff. peak/hole / e $\AA$ & $0.65 /-0.28$ \\
\hline
\end{tabular}



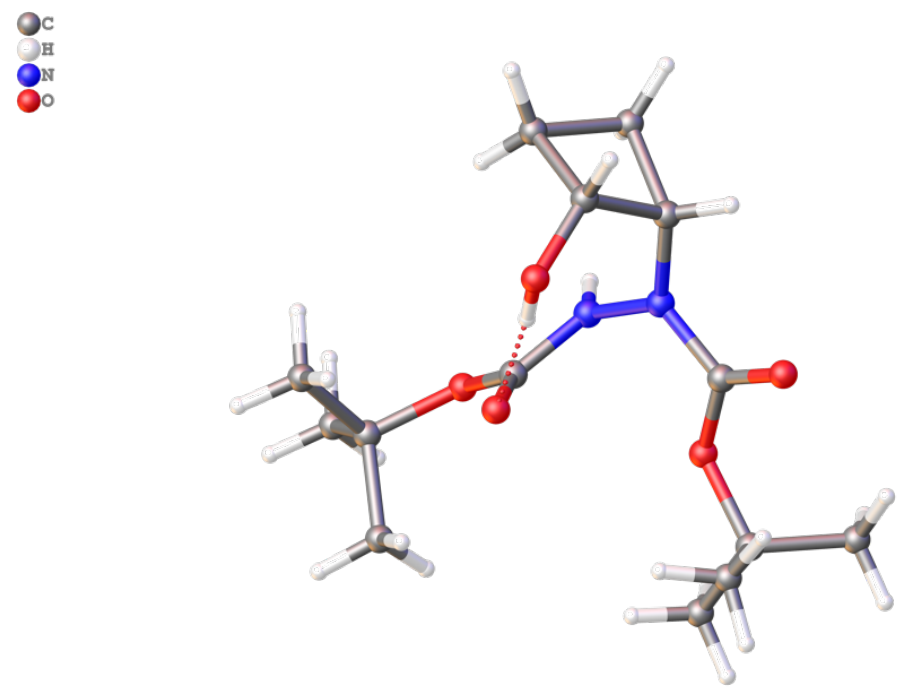

Identification code SC121

Empirical formula

$\mathrm{C}_{14} \mathrm{H}_{26} \mathrm{~N}_{2} \mathrm{O}_{5}$

Formula weight

302.37

Temperature/K

$100.00(10)$

Crystal system

monoclinic

Space group

$\mathrm{P} 2{ }_{1} / \mathrm{c}$

$\mathrm{a} / \AA$

10.58050(10)

$\mathrm{b} / \AA$

$10.87620(10)$

$c / \AA ̊$

15.39660(10)

$\alpha /{ }^{\circ}$

90

$\beta /^{\circ}$

98.9080(10)

$\mathrm{Y} /{ }^{\circ}$

90

Volume $/ \AA^{3}$

1750.40(3)

Z

4

$\rho_{\text {calc }} \mathrm{g} / \mathrm{cm}^{3}$

1.147

$\mu / \mathrm{mm}^{-1}$

0.718

$\mathrm{F}(000)$

656.0

Crystal size $/ \mathrm{mm}^{3}$

$0.301 \times 0.273 \times 0.142$

Radiation

$\operatorname{CuKa}(\lambda=1.54184)$ 
$2 \Theta$ range for data collection $/{ }^{\circ} 8.46$ to 153.16

Index ranges

$-13 \leq \mathrm{h} \leq 13,-13 \leq \mathrm{k} \leq 11,-19 \leq \mathrm{I} \leq 19$

Reflections collected

31493

Independent reflections

$3663\left[R_{\text {int }}=0.0330, R_{\text {sigma }}=0.0124\right]$

Data/restraints/parameters

$3663 / 0 / 197$

Goodness-of-fit on $\mathrm{F}^{2}$

1.058

Final $R$ indexes $[l>=2 \sigma(I)] \quad R_{1}=0.0425, w_{2}=0.1107$

Final $\mathrm{R}$ indexes [all data] $\quad \mathrm{R}_{1}=0.0433, \mathrm{wR}_{2}=0.1114$

Largest diff. peak/hole / e $\AA^{-3} 0.39 /-0.40$ 


\section{Differential Scanning Calorimetry (DSC) Trace for $\mathbf{2 d}$}

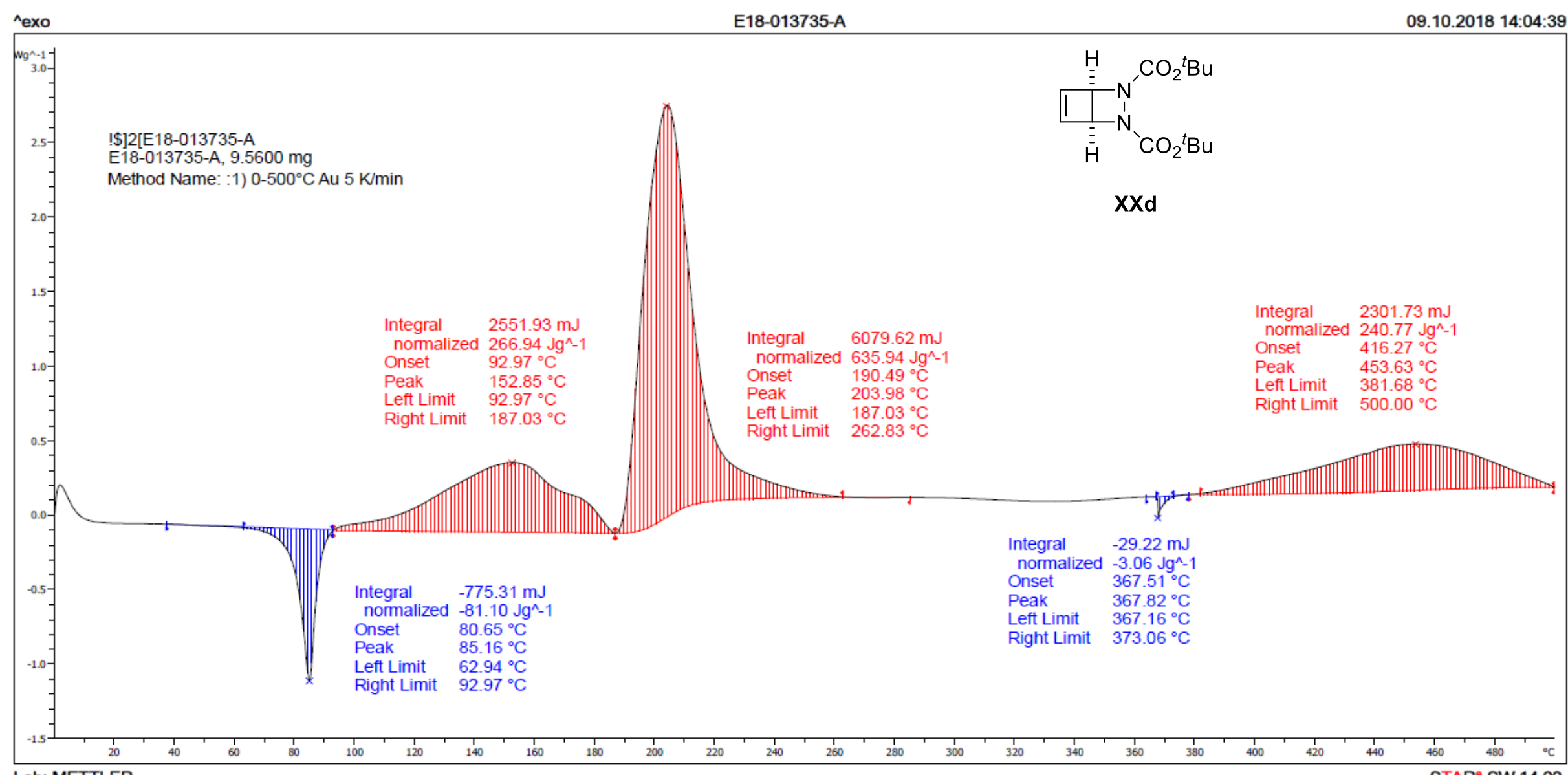


